\title{
Experimental Analysis of the Health and Well-being Effects of a Non-contributory Social Security Program
}

\author{
Emma Aguila (RAND), Arie Kapteyn (RAND), Rosalba Robles (Government of the \\ State of Yucatan), and Beverly Weidmer (RAND) ${ }^{1}$
}

Preliminary Version December 292010

\begin{abstract}
Non-contributory social security programs have been implemented in at least 15 countries around the world. These are cash transfer programs aimed at poverty alleviation among the elderly population. Previous studies have found that these programs reduce poverty and inequality, while the health effects are less clear.

Our study designs and evaluates a new non-contributory social security program in the State of Yucatan, Mexico. This program is for individuals 70 years or over. Eligible individuals are assigned to treatment and control groups and a large array of background variables and outcome measures are collected at baseline and during the course of the experiment for individuals in both the treatment and control groups. In the current paper we provide evidence of the impact of the program based on information collected six months after the implementation of the program in two cities in Yucatan selected for the first phase of the program that has a quasi-experimental design.

Even after this short period we find significant treatment effects on labor supply, hunger, medical consumption, and memory. Eligible individuals spend their pension on food, visits to the doctor, and medicines, while sharply reducing labor supply. We also find an increase in the daily number of cigarettes smoked and a decline in the consumption of alcoholic beverages.
\end{abstract}

JEL classification: J14, J26, I38

Keywords: Cash-transfer Programs, Elderly, Retirement

\footnotetext{
${ }^{1}$ We acknowledge the support from the State of Yucatan, Mexico; the RAND Corporation; the National Institute on Aging under grants P30AGO12815, R21 4-94-2008, and 5P01AG02248107. We would like to specially thank Yvonne Ortega, the Governor of the State of Yucatan for supporting this research program and Ulises Carrillo for his advice and support. We would also like to thank the advice and support of Orazio Attanasio, Lisa Berkman, Michael Hurd, Costas Meghir, Teresa Seeman, and James P. Smith. We are grateful to the field team in Yucatan, Mexico particularly Oscar Vargas, the team of interviewers, field supervisors, field coordinators, and administrative staff that worked in this stage of the project. We would like to thank Norely Martinez and Gabriela Castro for their excellent research assistance.
} 


\section{Introduction}

In many countries, poverty is more prevalent among the elderly than among younger groups of the population, particularly for the elderly who do not have access to social security benefits (Bourguignon et al., 2004). Social security programs for the elderly provide cash transfers, in-kind resources, and/or health interventions (Coady et al., 2003).

In this study we evaluate the impact of a non-contributory social security program for elderly on the health and well-being of recipients. Many countries have introduced non-contributory pensions, including Argentina, Bolivia, Brazil, Bangladesh, Chile, Kosovo, Mauritius, Namibia, Nepal, South Africa, and Zambia (e.g., Barrientos, 2006; Palacios and Sluchynsky, 2006; Farrington et al., 2006; Rofman, 2005). Most noncontributory pension plans define an eligibility age and many are means-tested. In Mexico, non-contributory social security schemes have been introduced by the federal government (70 y más) and by some states, including Mexico City, Zacatecas, Quintana Roo, Tabasco, Chiapas, and Yucatan.

Several studies have found that cash transfer programs targeting the elderly reduce poverty and inequality (for example Lund, 1993; Ardington and Lund, 1995; Lund, 1999; Case and Deaton, 1998; Leibbrandt, 2001; Delgado and Cardoso, 2000; Delgado and Cardoso, 2000b; Schwarzer, 2000; Schwarzer and Querino, 2002b). These studies have found improvements in health, enrollment rates of school-age children, housing, and access to credit, concentrating on outcomes of the program on household members but not specifically on the principal recipients due to the lack of information. Many studies use household information to compare beneficiaries with non-beneficiaries. To the extent that claiming benefits is a choice, doing so may introduce sample selection problems. To our knowledge, none of the studies have used program evaluation methods with an experimental design with treatment and control groups and measurements before and after the intervention.

This study presents the evaluation of the social policy intervention using a quasiexperimental design with rich data capturing health and well-being outcomes in old age. The outcome measures are largely similar to the ones measured in the Mexican Health and Aging Study (MHAS), which is very similar to the Health and Retirement Study (HRS) in the U.S.

The social security program we consider is designed for individuals 70 years old or older living in the State of Yucatan, Mexico and provides a flat rate pension equivalent to \$78USD per month at PPP (Purchasing Power Parity). The program is being introduced in phases with randomly assigned experimental and control groups. The research project started in October 2007. The first year of the project was devoted to developmental work including instrument development, multiple rounds of testing in the field, and recruitment and training of local field staff on computer assisted personal interviewing (CAPI), data collection protocols and procedures, and substantive training on the survey instrument. The survey instrument was designed in English and then translated into Spanish and Mayan. Two cities in the Northeast of the State of Yucatan, Valladolid and Motul, were selected for the first experimental phase of the implementation of the social security program. Valladolid was assigned to treatment and Motul to control. Prior to the announcement and implementation of the program in these two cities, we conducted a baseline survey of all the elderly adults age 70 or older, living 
in these two towns (August-November 2008). Eligible adults in the treatment town, Valladolid, started receiving the social security benefit in December 2008. The first follow-up surveys in both Motul and Valladolid were conducted in June and July 2009, approximately six months after the treatment town received the intervention. In this paper we report on the results from the baseline and the first follow-up survey in these two communities.

The comparison of Valladolid and Motul is only the first phase of the research study. Due to budget constraints, the social security program is being implemented in phases with a new phase rolled out every year. The second and third phases of the social security program are being implemented in Merida, the state capital and the largest city in the State of Yucatan. For both the second phase and third phases, city blocks were selected randomly and households were randomly assigned to treatment and control groups within blocks. Baseline surveys are conducted with all eligible adults (those 70 years old or older) in the blocks selected as part of this experiment. Baseline data collection was conducted between August and November 2009 for phase 2 and was conducted between August and November 2010 for phase 3. The implementation of the social security program in treatment blocks selected for phase 2 started in December 2009 and in December 2010 for phase 3. More phases are planned for later years.

The experimental design of the social security program offers unique opportunities to study the effect of income changes on a large number of outcomes. One such outcome of great interest is health. Establishing causal links between income and health is notoriously difficult. Although it is likely that causality runs both ways and that SES and health are likely influenced by common factors, assessing the strength of each of the sources (e.g., Smith, 1999) of correlation is challenging because of a lack of experiments (natural or otherwise). One possible approach to establishing causal relations is exploiting the timing of income/wealth changes and subsequent health changes, or vice versa timing of health shocks and their effect on income or wealth in later periods (e.g., Adams et al., 2003; Michaud and Van Soest, 2004; Smith, 2005). Generally, there seems to be a clear effect of health on wealth or income; the opposite effect appears weaker, at least in the U.S. for individuals 51 or older.

The issue of whether one can expect clear health effects as a result of the introduction of a social security benefits at advanced ages is open to question. One example suggesting that even at high ages health effects may be detectable is the experience of German reunification. This has been used as a natural experiment to look at the health and mortality trajectories of cohorts in East and West Germany before and after reunification (e.g., Frijters et al., 2005; Gjonça et al., 2000; Scholz and Maier, 2003). Scholz and Maier follow cohorts born in 1895, 1900, 1905, and 1910. Before unification, mortality in these cohorts was substantially higher in the East than in the West. After reunification, mortality converged quickly (in about 5-10 years), particularly for women. It is remarkable that even at older ages mortality may respond strongly to changing socioeconomic circumstances. Frijters et al. (2005) consider health satisfaction as measured in the German Socio-Economic Panel and find the income improvements in East Germany resulting from reunification had a significantly positive, albeit quantitatively small effect on health satisfaction.

Be that as it may, one may still ask how much of an effect one can expect to see after only six months. We consider a number of broad outcome categories: self-reported 
health, subjective mortality expectations, chronic and acute health conditions, ADL's and IADL's, smoking and alcohol consumption habits, depression, prevalence and intensity of pain, subjective wellbeing measures, food availability and food expenditures, paid work, doctor, dentist, and folk healer visits, number of outpatient procedures, some simple cognitive measures, food expenditure at home and out of home, as well as security and violence indicators. As will be seen, even after six months a number of clear effects emerge.

The initial results of the first phase of the experiment (conducted in Valladolid and Motul) show a positive effect on cognitive measures; a decline in hunger and deprivation of food, a decline in alcohol consumption, an increase in the daily number of cigarettes smoked, and a decline in labor supply. We find that individuals spend their cash transfer on food, visits to the doctor, and on buying medicines. More individuals report paying their out-of-pocket health expenses and a lower proportion report their relatives paying their out-of-pocket health expenses. The latter might indicate a crowding-out effect of family transfers to the elderly in terms of health expenses. We also find a lower proportion of elderly reporting not being able to travel to visit family or friends due to a lack of money. In the short term, the non-contributory social security program is causing a positive effect in the elderly population by decreasing hunger, increasing the amount of money they spend on food and medical expenses as well as reducing labor supply for those over 70 years old.

Although the social security program is limited to Yucatan, like other social experiments that have been carried out in Mexico, we expect to be able to draw inferences for other Mexican states and for both developing and developed countries. The remainder of the paper is structured as follows. Section II presents an illustrative model motivating the empirical analysis. Section III provides an extensive description of the data and of the organization of the fieldwork. Section IV provides a description of straightforward difference-in-differences analyses. Section V repeats the analysis of Section IV, but controls for a number of demographic variables and baseline socioeconomic status (SES) variables. We also present the results including interactions of treatment effects with baseline SES and gender. We test the robustness of the results comparing parametric and nonparametric estimates.

Since in this first phase we only compare two communities there may be concerns of community level cluster effects that would affect our results. We discuss these issues in Section II for the parametric specification, and again in Section VI, providing evidence suggesting that this is probably not of great concern. Section VI also discusses potential sources of bias and robustness checks. Section VII concludes with a summary of results and a preview of future experiments and data collection.

\section{A Model of Treatment Effects}

There are various issues in the design of the project that merit further discussion. The first issue is the possibility of cluster effects or aggregate changes taking place in either the treatment and control communities, or both. A second issue concerns possible spill-over effects from treated households to others.

Consider the following model:

$$
y_{i c t}=\mu_{i c}+v_{c t}+\gamma D_{i c t}+\delta D_{c t}+\varepsilon_{i c t}
$$


where:

$y_{\text {ict }}$ is an outcome variable of interest for household $i$ in town $c$ and time $t, t=1,2$

$\mu_{i c}$ is an individual effect for household $i$ in town $c$

$v_{c t}$ is a time effect in town $c$, representing any aggregate effect that may influence individual outcomes in a similar way.

$D_{i c t}$ is a treatment dummy: $D_{i c t}=1$ for the treated household if $t=2$; 0 otherwise

$D_{c t}$ is the spillover effect of treatments in town $c . B_{c t}=0$ if there is no treatment.

$\varepsilon_{i c t}$ is an iid error term independent of the other variables on the right hand side of the equation.

Note that there are no covariates; this is just done for simplicity; these can be added easily.

The nature of the spillover term $D_{c t}$ is not a priori clear. It can represent effects on the local provision of shops or facilities (now that there is more money to be spent by the elderly some services may open, for instance health clinics), or it can raise prices of goods that the elderly tend to buy. It may also reflect envy, or transfers from the recipients of a benefit to those who received nothing, etc. Several studies show that spillovers (or externalities) may be substantial (e.g. Angelucci and De Giorgi (2009), Miguel and Kremer (2003a), Kuhn, Kooreman, Soetevent, and Kapteyn (2010)) and may bias the measurement of treatment effects significantly if ignored.

Note that the experiment is meant to determine the effect of the introduction of a universal social security system. Thus, what we are after is really $\gamma+\delta \Phi_{c t}^{u}$, where $\square_{c t}^{u}$ represents the situation with universal social security coverage in the town.

Let's now consider identification of possible treatment effects. Denote a treated observation (in period 2) by a superscript $t$ and a non-treated observation by a superscript $n$. We consider two cases:

Case 1: Assume that in one community $T$ everyone gets treated and in a second community $N$ nobody gets treated. This is the case in the first phase of our experiment, where every eligible individual in Valladolid gets treated and no one in Motul. We then have for first differences:

$$
\begin{gathered}
\Delta y_{i T}^{t} \equiv y_{i T 2}^{t}-y_{i T 1}^{t}=\Delta v_{T}+\gamma+\delta \Delta \tilde{D}_{T}^{u}+\Delta \varepsilon_{i T}^{t} \\
\Delta y_{i N}^{n} \equiv y_{i N 2}^{n}-y_{i N 1}^{n}=\Delta v_{N}+\Delta \varepsilon_{i N}^{n}
\end{gathered}
$$

Difference-in-difference gives

$$
\Delta y_{i T}^{t}-\Delta y_{i N}^{n}=\Delta v_{T}-\Delta v_{N}+\gamma+\delta \Delta D_{T}^{u}+\Delta \varepsilon_{i T}^{t}-\Delta \varepsilon_{i N}^{n}
$$

So this identifies the full effect of the introduction of the social security program $\left(\gamma+\delta \boxplus_{c T}^{u}\right)$, plus a possible effect of different aggregate effects in the two towns $\left(\Delta v_{T}-\Delta v_{N}\right)$.

The term $\gamma+\delta \Delta \tilde{D}_{T}^{u}$ is the total treatment effect (own effect plus possible interactions/spillovers). For what follows, it is convenient to simply summarize that by a single parameter $\beta \equiv \gamma+\delta \Delta \tilde{D}_{T}^{u}$. The terms $\Delta v_{T}$ and $\Delta v_{N}$ can be thought of as fixed or random. If we think of them as fixed, the question arises if such aggregate movements are 
observable. As documented in Sections III and VI, we have collected various pieces of information at the community level through our community surveys, while other information (e.g. unemployment rates or the opening of a new hospital) can be obtained from public sources. It is possible therefore with considerable confidence, to decide if differences between Valladolid and Motul can plausibly be ascribed to other factors than the introduction of a Social Security program for the elderly (See section VI).

Nevertheless, it is conceivable that there are remaining aggregate effects ${ }^{2}$ that we don't observe. One possible approach is treating these effects as random. For a start let's simplify the notation in the equations above, to obtain:

$$
\begin{aligned}
& \Delta y_{i T} \equiv z_{i T}=\alpha+\beta+u_{i T}+\eta_{T} \\
& \Delta y_{i N} \equiv z_{i N}=\alpha+u_{i N}+\eta_{N}
\end{aligned}
$$

where $u_{i T} \equiv \Delta \varepsilon_{i T}^{t}$ and $u_{i N}=\Delta \varepsilon_{i N}^{t}$ are assumed i.i.d. with variance $\sigma_{u}^{2}$ and the aggregate errors $\eta_{T}$ and $\eta_{N}$ are i.i.d. with variance $\sigma_{\eta}^{2}$. The common intercept $\alpha$ represents the assumption that, except for the random errors $\eta_{T}$ and $\eta_{N}$, observable aggregate movements are the same across the two communities (that is: $\Delta v_{T}=\alpha+\eta_{T}$ and $\Delta v_{N}=\alpha+\eta_{N}$ ). Furthermore, for notational simplicity let's assume that in both towns we have exactly $m$ observations. We can estimate the parameter of interest $\beta$ by means of OLS. The estimate for $\beta$ is simply $\beta=\bar{Z}_{T}-\bar{Z}_{N}$, i.e. the difference in sample means between the treatment town and the control town (remember that $z_{i T}$ and $z_{i N}$ are first differences, so we estimate $\beta$ as the difference in changes, as in equation (1) above).

It is easy to work out the variance of the estimator (see e.g. Moulton (1990)):

$$
\operatorname{var}(\beta)=2 \frac{\sigma_{u}^{2}}{m}+2 \sigma_{\eta}^{2} .
$$

This result is intuitive. The variance of each of the individual means $\bar{Z}_{T}$ and $\bar{Z}_{N}$ is equal to $\sigma_{u}^{2} / m$ so the variance of their difference, conditional on $\eta_{T}$ and $\eta_{N}$ is $2 \sigma_{u}^{2} / m$. In addition the variance of the difference of the independent aggregate errors $\eta_{T}$ and $\eta_{N}$ is equal to $2 \sigma_{\eta}^{2}$.

Clearly by having only two communities in the first phase, we cannot control for differential aggregate changes in these two communities in a statistical way. We have to assume we can observe them, or we have to assume they are small enough not to swamp the treatment effects we are after. If we treat them as observable, we may want to assume (or verify to the extent possible) that the differences across towns are small. If we want to allow for the fact that not everything is observable, then the issue is how large the unobserved component is. If, for instance, we want to base conclusions on t-statistics then these two points are similar for practical purposes. Consider the "naïve" t-statistic ignoring aggregate effects

\footnotetext{
${ }^{2}$ Or a clustering effect: A convenient way to represent clustering effects is to allow for equi-correlated errors (see e.g. Moulton (1990), which is equivalent to the approach here.
} 


$$
t=\frac{\bar{Z}_{T}-\bar{Z}_{N}}{\sqrt{2 \frac{s_{u}^{2}}{m}}}
$$

Allowing for clustering effects implies that we should increase the term under the square root with some upper bound on $2 \sigma_{\eta}^{2}$. Allowing for differential aggregate effects means that we should reduce the absolute value of the numerator by some upper bound on the absolute value of the differential aggregate effect $\Delta v_{T}-\Delta v_{N}$. In both cases we would lower the t-statistic and thus be conservative in deciding that an observed difference is statistically significant.

We should realize that we are talking about unobservable effects in changes. Due to first differencing any correlation in levels within a town is eliminated.

Case 2: Assume that in each town $c$ we have both treated and non-treated households. This is the case in phase 2 of the social security program, where we randomly assign treatments and controls in Merida, the capital city of the state and also the largest town. Then we have:

$$
\begin{gathered}
\Delta y_{i c}^{t} \equiv y_{i c 2}^{t}-y_{i c 1}^{t}=\Delta v_{c}+\gamma+\delta \Delta D_{c}+\Delta \varepsilon_{i c}^{t} \\
\Delta y_{i c}^{n} \equiv y_{i c 2}^{n}-y_{i c 1}^{n}=\Delta v_{c}+\delta \Delta D_{c}+\Delta \varepsilon_{i c}^{n}
\end{gathered}
$$

Where the $\Delta$ once again indicates first differences. Now consider dif-in-dif:

$$
\Delta y_{i c}^{t}-\Delta y_{i c}^{n}=\gamma+\Delta \varepsilon_{i c}^{t}-\Delta \varepsilon_{i c}^{n}
$$

So this identifies $\gamma$, but as argued above, it misses any spillover effect.

The models considered here are simple, but they illustrate the main point. Implementing universal coverage in a small number of towns takes care of possible spillover effects, but implies vulnerability to aggregate changes that are different in the treatment and control towns, or to cluster effects or aggregate effects that are not completely observable. On the other hand, complete randomization within a town cannot identify the spillovers correctly for two reasons: (1) $D_{c t}^{u}$ and $D_{c t}$ may be very different and actually their effects may be different. For simplicity we have assumed a linear effect and a common parameter $\delta$, but one can easily imagine non-linearities where the own treatment interacts with $D_{c t} ;(2)$ as noted, dif-in-dif only identifies $\gamma$.

The strength of the current project is that we consider both designs: (1) designs where entire communities get assigned to the treatment group and (2) designs where randomization to treatment or control occurs within a city. In the second case we could randomize at the individual level, but randomization across blocks provides us with an opportunity to measure the extent of spillovers by modeling the effect on non-treated households in a way similar to that adopted by Miguel and Kremer (2003a). We will know the exact location of all treated and non-treated households (we collect the geographic coordinates for each household) and can use the geographical distance between households as a measure of the likely intensity of spillovers and thus ascertain the importance of these. Having both designs where an entire community is treated and another where treatment varies within a town should create opportunities to gain more 
insight than would be available from just one of the two designs (either randomization within a town or treating a whole town).

\section{First Phase of the Experiment}

The social security program being implemented in the State of Yucatan is called Reconocer Urbano, a non-contributory, universal pension program being implemented in phases throughout the state. To avoid overlapping with other Federal government programs that are being implemented in localities with less than 20,000 inhabitants, the third phase of Reconocer Urbano is being implemented in 11 municipalities with more than 20,000 inhabitants. The roll-out of the pension program is done in stages. The first phase started in Valladolid in December 2008 with 1,047 beneficiaries.

The research study conducted to evaluate the impact of the pension program is called Escuchar and involves a series of surveys that collectively are dubbed ENCAHEY (Encuesta de Características Socioeconómicas del Hogar en el Estado de YucatánSurvey of Household Socioeconomic Characteristics in the State of Yucatan).

\section{III.1 Development of the Survey Instrument}

Development of the baseline survey instrument started in the fall of 2007. In developing the baseline survey, we included survey measures that have been validated and tested in other surveys in both English and Spanish. Items included in the survey instrument were taken or adapted from existing surveys used in other longitudinal studies including the Mexican Health and Aging Study, the US Health and Retirement Study, the New Immigrant Study, Oportunidades, as well as from various family life surveys, some of them conducted by RAND.

\section{III.2 Survey Content}

The survey instruments used as part of Escuchar collect detailed community, household, and individual-level data at baseline (before the program is announced or implemented) and then yearly with the first follow up interview in both treatment and control groups approximately six months after the treatment group receives the intervention (the first pension payment). Both baseline and follow up surveys collect selfreported data on health, depression, chronic conditions, activities of daily living (ADLs), instrumental activities of daily living (IADLs), physical functioning, anthropometric measurements and a number of biomarkers. We collect anthropometric measurements for every age-eligible respondent, including height, weight, waist circumference, arm circumference, arm length, and height to knee. We also collect blood pressure, lung capacity, grip strength and do a series of balance tests and a timed walk. Where appropriate, respondents are asked for continuous answers (e.g., when asked for monetary quantities). If the respondent is unable to answer, unfolding brackets are used to reduce the number of missing responses. This mimics the current practice in the HRS.

\section{III.3 Translation}

In February and March of 2008, we updated the translation of the Spanish version of the survey instrument, and translated the survey instrument into Mayan. All survey instruments used in the evaluation study are available in Spanish, Mayan, and English. 
Data collection is conducted in both Spanish and Mayan. Most of the interviews are conducted in Spanish; however, a significant percentage is conducted in Mayan.

\section{III.4 Sampling Methods}

To build the sampling frame for this study, we first carry out a complete listing of all households in a selected community and screen them in order to identify households with eligible adults. We have signed a collaborative agreement with INEGI, the National Institute for Statistics and Geography (the federal agency responsible for conducting the population census in addition to many other surveys) whereby they provide us with maps of the communities selected for each phase of Reconocer Urbano, and update these maps as necessary (a cartographer accompanies our data collection team to selected communities and updates the maps as households are being listed)

We currently have five data collection teams comprised of one Field Supervisor and 6-7 interviewers. Our Survey Coordinator in conjunction with the field supervisors has day-to-day responsibility for implementing the household survey, logistics and quality control. June and July of 2008, we conducted a census of all the households in Valladolid and Motul. In addition, we screened each household to identify those with age eligible respondents. This created the sampling frame for the baseline survey of the evaluation study. Table 1 shows results to date of the field operations for the first phase of the evaluation study. In Valladolid, we listed a total of 15,535 households and identified 2,371 persons age 65 years or older. In Motul, we listed 7,328 households and identified 1,547 persons age 65 years or older. In total for the first phase of the evaluation study, 22,863 households were listed, and 3,918 individuals 65 years old or older were identified and listed.

\section{III.5 Baseline Data Collection for the First Phase}

Baseline data collection in Valladolid took place in August and September 2008. Baseline data collection in Motul took place in October and November 2008. After the collection of baseline data, Reconocer Urbano was implemented in Valladolid in December 2008. The first follow-up interview in both Valladolid and Motul was completed simultaneously in the summer of 2009.

\section{III.6 Community Survey}

In February and March 2009, we completed key informant interviews with government officials in Valladolid and Motul. The Community Survey, adapted from the Community Survey used in the Indonesian Family Life Survey collects information about local transportation, electrical infrastructure, water and sanitation infrastructure, community history, migration, housing, business resources and infrastructure, environment, economic activity and employment, and community-level finance, etc.

\section{Initial Findings}

Our approach is probably closest to that of Case (2001), who considers the health effects of the dramatic expansion of the South African pension system. Her analysis is based on a comparison of households that did and did not have members receiving the 
pension. Moreover, the data indicate whether income is pooled among household members. She found that in households where income is pooled, pension receipt helps to improve the health status of all household members. She also discusses several mechanisms through which this may have happened and finds evidence for various pathways, including improved sanitation, nutrition (fewer skipped meals), and mental health.

One may wonder how large an effect can possibly be found over merely a six months period. As noted above, Scholz and Maier (2003) find that even among the oldest-old, health may be affected substantially over a relatively short period. Thus, if the pension has a substantial role to play in improving health among receiving households, then we expect at least some significant effects over a relatively short time period.

In Table 2 we present the descriptive characteristics of the baseline sample from the two communities, Valladolid and Motul, selected for the first phase of Reconocer Urbano. A high proportion are married or widowed as expected. Most individuals have no schooling or only incomplete primary education. Over 70 percent of the persons 70 years or older speak Mayan. A high proportion of individuals, over 40 percent, are illiterate as they report not being able to read and write a message in Spanish in Valladolid and over 30 percent in Motul. It is worth highlighting that more than 10 percent report living alone. This seems to be a quite high number given that in Mexico the elderly traditionally live with their children.

Table 3 shows simple difference-in-differences of the means for the main outcome variables. We observe the following patterns:

- The number of acute conditions declined more in Valladolid than in Motul. We define as acute conditions heart attack, stroke, liver or kidney infection, tuberculosis, and pneumonia. We find a decline by $22 \%$ in the treatment group.

- Health satisfaction improves in both towns, but more in Valladolid.

- Satisfaction with life as a whole also improves more in Valladolid than in Motul, but the difference is not significant.

- The number of respondents that drink alcohol beverages in the control town Motul increased but it remained constant for the treatment town.

- The number of drinks per day consumed decreased in Valladolid and increased in Motul.

- The number of respondents in Valladolid paying a visit to a doctor in the previous three months increased from 41 to 52 percent, while in Motul it increased from 48 to 50 percent. In Valladolid in comparison to Motul, we find an increase by $21 \%$ in the number of individuals that report visiting the doctor.

- The number of doctor visits fell in Motul and increased in Valladolid. The number of visits to the doctor increased in Valladolid by $27 \%$.

- The number of instances where respondents report not to have taken medications because of the cost of the medication shows significant improvement in Valladolid. We find a decline by $25 \%$ on individuals reporting not buying medicines because there were too expensive.

- The out-of-pocket expenses for medical costs or medications paid by relatives declined in Valladolid and remained constant in Motul. In fact, the out-of-pocket 
expenses paid by the elderly eligible of the program increased in the treatment town and the proportion remained constant in the control group.

- We find a lower proportion of individuals in Valladolid reporting not having enough money to visit family or friends in comparison to the control group.

- The number of instances that respondents reported not having enough food ("How often in the last 3 months did you run out of food and you didn't have the money or resources to get more”) decreased significantly in Valladolid.

- $\quad$ Skipping or cutting meals (How often in the past 3 months, did you or another person in your household ever cut the size of your meals or skip a meal because there wasn't enough money to buy food) has become significantly less prevalent in Valladolid than in Motul.

- $\quad$ Eating less than needed (How often in the past 3 months, did you or another adult in your household eat less than you felt you should because there wasn't enough money to buy more food?) declined in Valladolid much more than in Motul.

- Elderly in Valladolid are less often hungry. This corresponds to the question: How often in the past 3 months, were you or other adults in your household hungry but didn't eat be cause you couldn't afford enough food?

- The incidence of not eating a day because of lack of food decreases significantly in Valladolid relative to Motul (How often in the past 3 months, did you or another person in your household not eat all day because there wasn't enough money to buy food?)

- We find less elderly are obtaining emergency food in Valladolid captured in the question: How often in the past 3 months, did you or another person in your household get emergency food from a church, government institution, or other institution?

- $\quad$ The amount spent on food last week increases slightly in Motul, but goes up by 31 pesos per week in Valladolid. In view of the pension of 550 pesos per month, this suggests that about 26 percent of the pension is spent on an increase in food consumption.

- The number of respondents in Valladolid reporting to have worked for pay in the last month fell from 16 to 12 percent, while in Motul it stayed the same (16 percent). There is thus a decline by $27 \%$ in work for pay for the treatment group.

- In terms of cognitive capabilities, immediate and delayed recall of words improved in Valladolid and declined in Motul. We find an increase in immediate recall by $13 \%$ and $29 \%$ in delayed recall for the treatment town.

\section{Treatment Effects while Controlling for Covariates}

Tables 4a-4i present results of the following model, which is a slightly simplified version of the model presented in Section 2:

$$
y_{i c t}=\mu_{c}+v_{t}+\gamma D_{i c t}+X_{i c t}^{\prime} \beta+\varepsilon_{i c t}
$$

where:

$y_{\text {ict }}$ is an outcome variable of interest for household $i$ in town $c$ and time $t, t=1,2$ 
$\mu_{c}$ is a town dummy

$v_{t}$ is a time dummy

$D_{\text {ict }}$ is a treatment dummy: $D_{\text {ict }}=1$ for the treated household if $t=2 ; 0$ otherwise

$X_{\text {ict }}$ is a vector of individual or household characteristics

$\varepsilon_{i c t}$ an error term independent of the other variables on the right hand side of the equation.

Since age is included as a quadratic we have included a row in the table indicating the maximum or the minimum (as the case may be) of the age parabola for each equation. Results of F-tests are included for the age parabola, education dummies, and for the quartile dummies for baseline personal income and household wealth. We have used linear regression, Tobit, Probit, and ordered Probit as appropriate. Tables 6a-6d show these results using probit, and ordered probit models for binary response outcome variables and ordered variables, respectively. We use Tobit models for the expenditure variables as we have a truncated distribution due to infrequency of purchase.

The response categories vary by question and sometimes a minus sign may imply an improvement, whereas in other cases it would indicate deterioration. To ease interpretation of the outcomes, the headings indicate the response categories.

\section{V.1 Health}

Tables 4a to $4 \mathrm{e}$ presents a number of health and subjective well-being related outcomes. The row "Valladolid wave 2 " shows the treatment effects of the social security program after six months. There does not appear to be a discernible effect on the number of chronic or acute health conditions, nor on the number of ADL's or IADL's. As in Table 3 we see an increase in the number of doctor's visits and a decrease in the number of cases where a household foregoes the use of medicine because it would be too expensive. Tables $4 \mathrm{~d}$ and Table $4 \mathrm{e}$ also show that more individuals report paying their out-of-pocket health expenses and fewer reports that their relatives pay their out-ofpocket health expenses.

The effects of the demographics are according to expectation. ADL's and IADL's go up with age, but not significantly. Doctor's visits increase with age (the top of the parabolas are at 60 years for the prevalence of doctor's visits and at 70 for the number of doctor's visits). The number of chronic and acute conditions seems to go up until approximately age 80 and to decrease after that. Individuals with completed primary schooling have fewer IADL's.

\section{V.2 Satisfaction}

Table 4a and Table $4 \mathrm{~b}$ show the determinants of satisfaction in six domains. The treatment effects are not significant, although there is some suggestion that satisfaction with income, health, and life in general has improved. 


\section{V.3 Alcohol Consumption and Smoking Habits}

Table 4c shows a decline in the number of alcoholic beverages consumed and the number of drinks consumed per day. The coefficient for the number of cigarettes smoked per day is positive but not significant.

\section{V.4 Food Expenditure, Food Availability, and Eating Patterns}

The last three columns of Table 4f, Table 4g, and Table 4h summarize the effects of the social security program on food availability, food consumption, and eating patterns. The treatment outcomes for the first two columns of Table $4 \mathrm{~g}$ show a remarkable contrast. The response to the question "How often in the last 3 months have you been worried that food would run out before you got money to buy more" suggests an increase in prevalence. On the other hand, the response to the question "How often in the last 3 months did you run out of food and you didn't have the money or resources to get more" indicates a clear improvement. Thus, the households in the treatment group seem to worry more at the follow up, while at the same time the actual incidence of food shortage has decreased. The effect is not statistically significant for being worried that food would run out before you got money to buy more. Table $4 \mathrm{~g}$ also shows an overall improvement as a result of the treatment: individuals in Valladolid are less frequently hungry, less likely to eat less than they felt they should, less likely to have gone a full day without eating and are less likely to have received food from a charity. Although not significant, the estimates for the amount of money spent on food last week, suggests an increase of about 24 pesos a week, or about 96 pesos a month (see Table $4 \mathrm{~h}$ ). This would suggest respondents spend about one sixth of the pension on an increase of food at home. This estimate is somewhat smaller than suggested by the simple dif-in-dif results in Table 3. On the other hand, the table also suggests a decrease in the amount spent on food away from home.

Education generally has a highly significant effect on the prevalence of hunger and the amounts spent on food.

\section{V.4 Labor Supply and Cognitive Functioning}

Table 4i shows a strong treatment effect on whether a respondent worked for pay last month. The estimate implies a reduction of work for pay by 4.3 percentage points. On a base of about $16 \%$ in wave 1 , this implies a reduction by about $30 \%$. Strikingly, the treatment has a significant positive effect on memory; both immediate and delayed word recall show significant improvements. This may be related to the improved nutritional situation described above.

\section{V.5 Interactions}

The current social security benefit is not means-tested. One would expect the benefit to have more of an effect for households with limited financial means than for households that were already well off before the benefit receipt. To investigate this further we have rerun the analyses in Table 4 while including interactions with income quartile and with gender. Table 5a-5i presents the results. For simplicity we only present the interactions and the results of the significance tests. The results for the other variables don't change much compared to Tables 4a-4i. Using probit, ordered probit, and Tobit 
models as in Tables 6a-6i, the results of the interactions are qualitatively similar. Tables 7a-7i show the results including the interaction terms using discrete choice models.

The income and wealth quartiles refer to the baseline wave. To preserve a maximal number of observations we have followed the strategy of including a dummy for missing observations for baseline income or baseline wealth. The dummies for missing information are taken to be the reference category for these cases.

Table 5a does not show significant interaction effects of the treatment with personal income. Males become significantly less likely to report that they ran out of food, skipped or cut meals, food was not always enough, and went hungry. This is confirmed by Table $5 f$ and $5 g$, where generally males report marked improvements in food availability and reduction of the incidence of hunger. Males increase their expenditure on food at home, which explains the results on the food availability variables. There do not appear to be gender or income differences in the effect on immediate or delayed word recall. The effect on work effort is larger for males and there is a significant effect on work effort. In the sample, $5 \%$ of women and $28 \%$ of men work. We find a decline by $25 \%$ in the proportion of men that work.

So, perhaps somewhat surprisingly the only interaction effect we find is with gender. Baseline income does not play a significant role, except for work effort.

\section{Discussion}

In this section we discuss potential sources of bias of the difference-in-differences estimates. Some of these are differential macro shocks, treatment announcement effects, differential implementation of the government programs in the treatment and control towns, and differences in pre-treatment trends. We also discuss the validity of the estimates and potential bias due to cluster effects.

\section{VI.1 Announcement Effects in Treatment Group}

We have designed the rollout schedule of the program and the timing of public information campaigns designed to promote the program jointly with the State government of Yucatan. This level of input into the design of the program is allowing us to limit anticipatory effects that have been observed in other programs and spillover effects that could occur from the program being implemented in treatment and control groups that are geographically proximate.

Because of funding constraints and competing priorities, the program will be implemented in stages across the 11 towns in Yucatan with a population of more than 20,000. Control groups will remain untreated at least until the end of the rollout period scheduled for mid-2012 and will not receive information about when they will receive the intervention until right before the program will be extended. Originally the plan was to incorporate all control groups into the program by the end of 2012. In view of budget constraints due to the recent economic crisis, it now seems likely that the end date will be moved back, allowing us to follow control and treatment groups over a longer period. 


\section{VI.2 Differential Implementation of Government Programs in Treatment and}

Control Groups

In June 2007, the Mexican federal government announced a social security program for elderly residents 70 and older (called 70 y Más) living in towns with less than 2,500 inhabitants. In February 2008, the program was expanded to cover towns with less than 20,000 inhabitants. To complement this federal program, the state of Yucatan initiated a new social security program for the elderly that is implemented in the eleven towns and cities of more than 20,000 inhabitants. There is no overlap between the federal government program and the state level program. Moreover, the State of Yucatan agreed to ensure there would be no differential implementation of other public programs in these towns.

\section{VI.3 Differences in Pre-treatment Trends for Treatment and Control Groups}

According to the Mexican Census 2005, Valladolid had a total of 45,868 inhabitants and Motul 21,508 inhabitants. The Census 2005, also reports that 10.95 percent of the population 15 years old or above are illiterate in Valladolid and in Motul this figure is 11.23 percent. Table 8 shows other indicators of poverty at the community level. Valladolid is categorized according to the Census 2005 as having a low level of poverty and Motul has a medium level. However, it should be noted that the poverty index between Valladolid and Motul is not that different: they are both on the margin between low and medium level of poverty. Motul has a higher proportion of households without sewage or toilet and piped water than Valladolid. All other indicators are similar between the treatment and the control town.

We conducted a community survey to understand in more detail the differences between the two cities in terms of health care infrastructure, economic activity, government programs, among others. None of the towns have flooding problems, they have enough public light systems, and the air quality is good. Both towns have similar federal government programs and state government programs. In terms of health infrastructure both towns have clinics of the Mexican Social Security Institute (IMSS), and the Ministry of health of the Government of the State of Yucatan. Valladolid has 6 clinics of the Ministry of health and Motul has only one. Also Valladolid has 4 private hospitals and Motul has none. All other health infrastructure is similar. The economic activity in both towns includes manufacturing industry (textile, automotive, wood, plastic, etc.), assembly plants, construction, wholesale and retail commerce, restaurants, and hotels. In addition, Valladolid has some agricultural employment. The most important economic activities in Motul are the assembly plant, construction, and services (commerce, restaurants, and hotels). For Valladolid the most important activities are services, manufacturing, and agriculture.

In sum, Valladolid has more inhabitants; therefore there is a larger infrastructure of services. The poverty index in Motul is slightly higher. They receive similar federal and state government programs. In terms of economic activity, they overlap in most of them but Valladolid has also agriculture.

\section{VI.4 Differential Macro Shocks in Treatment and Control Groups}

In the community survey, we also collect information about aggregate changes in the treatment and control towns. In terms of natural disasters, none of the two cities has 
experienced floods, earthquakes, fires, landslides, hurricanes or plagues since the beginning of the social security program. However, both towns have had droughts (April and May 2009). In Valladolid, officials reported that the drought affected 22 persons and Motul did not report any effects. This suggests only small differences in aggregate effects between the two towns. In general the state of Yucatan suffered a drought in April and May 2009. Since one of Valladolid's economic activities is agriculture, we would expect that if anything aggregate effects have been more severe in Valladolid.

\section{VI.5 Robustness Checks and Sensitivity Analysis}

Reported standard errors allow for clustering at the household level. Since we include a town dummy, systematic aggregate differences are controlled for. As argued above, differential aggregate changes between the two towns are probably small and more likely to have affected Valladolid than Motul. Nevertheless, further sensitivity analyses are still possible. For now, we show the results using propensity score matching. Figure 1 presents the densities of the propensity score for the treatment and control groups. We can observe a common region of support between groups. After imposing the common support we drop $1.04 \%$ of the sample. The propensity core is estimated using a probit model that estimates the probability of being treated conditional on the demographic characteristics included as control variables in tables $4 \mathrm{a}-4 \mathrm{~d}$. Therefore, we can compare the propensity score matching results to the parametric findings. We tested the balancing property and it is satisfied (Rosenbaum and Rubin, 1983).

Table 9 shows the results comparing the difference-in-differences of the mean, using parametric and non-parametric methods for the outcome variables for which we found an effect of the program in previous sections. We obtain very similar coefficients and standard errors with propensity score matching as in the OLS regressions.

\section{VI.6 Attrition}

In the baseline survey of Valladolid we obtained 1,264 interviews and 956 in Motul (see Table 10). For the follow-up the re-interview rate for Valladolid was 91.6 percent and 89.8 percent for Motul. Failure to re-interview was due to death, refusal or non-contacted; 5 percent of the sample from Valladolid died and 5.9 percent in Motul; 0.7 percent of the sample refused to do the follow-up survey in Valladolid and 1.2 percent in Motul. We were unable to contact 2.5 percent of the individuals in Valladolid and 2.9 percent in Motul. We will do further analysis to understand the characteristics of the different groups that refused or could not be contacted in order to understand potential attrition bias. However, the number of refusals or non-contacts are relatively small so that we anticipate negligible attrition effects. 


\section{Conclusion}

In this study we have presented initial results of an experimental research project that designs and evaluates the impact of a non-contributory social security program as a poverty alleviation policy for the elderly. We analyzed the effects of the program on the health and well-being of elderly recipients, using data from the first phase of the experiment. We also described the evaluation design, capacity building efforts, survey instrument, and field operations.

The initial results are a decline in hunger, cutting meals, and running out of food due to the lack of money. The elderly recipients are spending more on food, visits to the doctor, and medicines and show improvement in memory function. We also find a decline in alcohol consumption. There is some indication of improved subjective wellbeing and a decline in acute conditions. For many of the effects we would expect substantially larger effects over a longer period. The second set of follow-up surveys is planned for the beginning of 2011. By then the program will have been in existence for about two years so that we can see if the initial findings get confirmed, become more pronounced, or get weaker.

Simultaneously with the follow-up studies in Valladolid and Motul, we are conducting several new experiments in Merida. By combining different sampling schemes and by considering variations on the basic experiment (e.g. providing benefits in the form of debit cards, additional health experiments, targeting experiments, etc.) the project should not only provide insight in the effects of a non-contributory social security scheme, but also in the effect of various parameters that may affect the efficacy of alternative designs. 


\section{References}

Adams, P., M.D. Hurd, D. McFadden, A. Merril, and T. Ribeiro (2003), "Healthy, Wealthy, and Wise? Tests for Direct Causal Paths between Health and Socioeconomic Status.” Journal of Econometrics 112, 3-56.

Aguila, E., R. Robles, and O. Vargas (2010), "Annual Report Escuchar Program” Government of the State of Yucatan, Mexico, 1--200.

Angelucci, M. and G. De Giorgi (2009), "Indirect Effects of an Aid Program: How Do Cash Transfers Affect Ineligibles’ Consumption?”, American Economic Review 99, 486-508.

Ardington, E. and F. Lund (1995), "Pensions and Development: Social Security as Complementary to Programmes of Reconstruction and Development." Development Southern Africa 12, 557-577.

Barrientos, A. (2006), “Cash Transfers for Older People Reduce Poverty and Inequality.” Draft Background Paper for World Development Report: Equity and Development.

Bourguignon, F., M. Cicowiez, J.J. Dethier, L. Gasparini, and P. Pestieau (2004), "What Impact Would a Miniumum Pension Have on Old Age Poverty? Evidence from Latin America." Paper presented at the Keep the Promise of Old-age Security Conference, Bogotá, Colombia, June 22-23.

Case, A. (2001), "Does Money Protect Health Status? Evidence from South African Pensions." In: D. A. Wise (ed.) Perspectives on the Economics of Aging, University of Chicago Press, 287-311.

Case, A. and A. Deaton (1998), “Large Cash Transfers to the Elderly in South Africa.” The Economic Journal, 108, 1330-1361.

Coady, D., M. Grosh, and J. Hoddinott (2003), “Targeted Anti-poverty Interventions: A Selected Annotated Bibliography.” World Bank, Washington, D.C.

Consejo Nacional de Población (2005), Municipales y Estatales, c. 2005. As of June 1, 2010:http:/www.conapo.gob.mx/index.php?option=com_content\&view=article\&id= 126\&Itemid=204

Delgado, G.C. and J.C. Cardoso (2000), “Condicões de Reproducão Econômica e Combate à Pobreza.” In: G.C. Delgado and J.C. Cordoso (eds.) A Universalização de Direitos Sociais no Brazil: a Prêvidencia Rural nos anos 90, Brasilia: IPEA.

Delgado, G.C. and J.C. Cardoso (2000b), "Principais Resultados da Pesquisa Domiciliar Sobre a Previdência Rural na Região Sul do Brasil.” Texto para Discussao 734, Rio de Janeiro: Instituto de Pesquisa Econômica Aplicada.

Farrington, J., J.P. Harvey, and R. Slater (2006), "Cash Transfers in the Context of Pro-Poor Growth.” Discussion paper for OECD/DAC Povnet Risk and Vulnerability Task Group.

Frijters, P., J.P. Haisken-DeNew, and M.A. Shields (2005), “The Causal Effect of Income on Health: Evidence from German Reunification.” Journal of Health Economics 24, 997-1017. 
Gjonça, A., H. Brockmann, and H. Maier (2000), “Old-Age Mortality in Germany Prior to and After Reunification.” Max-Planck-Institute for Demographic Research, Demographic Research 3/1.

Kuhn, P., P. Kooreman, A.R. Soetevent, A. Kapteyn (2010), "The Own and Social Effects of an Unexpected Income Shock: Evidence from the Dutch Postcode Lottery”, American Economic Review, forthcoming

Leibbrandt, M. (2001), "Household Incomes, Poverty and Inequality in a Multivariate Framework.” In: H. Bhorat, M. Leibbrandt, M. Maziya, S. van der Berg, and I. Woolard, (eds.). Fighting poverty. Labour Markets and Inequality in South Africa, Cape Town.” UCT Press: Cape Town, South Africa, 130-154.

Lund, F. (1993), “State Social Benefits in South Africa.” International Social Security Review 46(1), 5-25.

Lund, F. (1999), "Understanding South African Social Security Through Recent Household Surveys: New Opportunities and Continuing Gaps.” Development Southern Africa 16(1), 5567.

Michaud, P-C. and A. van Soest (2004), "Health and Wealth of Elderly Couples.” RAND working paper, WR-191.

Miguel, E. and M. Kremer (2003a), "Worms: Identifying Impacts on Education and Health in the Presence of Treatment Externalities.” Econometrica 72, 159-217.

Palacios, R. and O. Sluchynsky (2006), “The Role of Social Pensions.” Pension Reform Primer

Rofman, R. (2005), “Social Security Coverage in Latin America Social Protection.” Discussion Paper No. 0523). World Bank, Washington, D.C.

Scholz, R. and H. Maier (2003), "German Unification and the Plasticity of Mortality at Older Ages.” Max-Planck-Institute for Demographic Research, Working Paper WP 2003-031.

Schwarzer, H. (2000), "Impactos Socioeconômicos do Sistema de Aposentadorias Rurais no Brazil - Evidências Empíricas de un Estudio de Caso no Estado de Pará.” Discussion Paper 729, Rio de Janeiro: IPEA.

Schwarzer, H. and A.C. Querino (2002b), “Non-Contributory Pensions in Brazil: The Impact on Poverty Reduction.” ESS Paper 11, Geneva: Social Security Policy and Development Branch. ILO.

Smith, J.P. (1999), "Healthy Bodies and Thick Wallets: The Dual Relationship between Health and Economic Status.” Journal of Economic Perspectives 13, 145-166.

Smith, J.P. (2005), “Consequences and Predictors of New Health Events.” In: D.A. Wise (ed.) Analyses in the Economics of Aging, University of Chicago Press, 213-239. 


\section{Figure 1.- Densities Propensity Scores for Treatment and Control Groups}

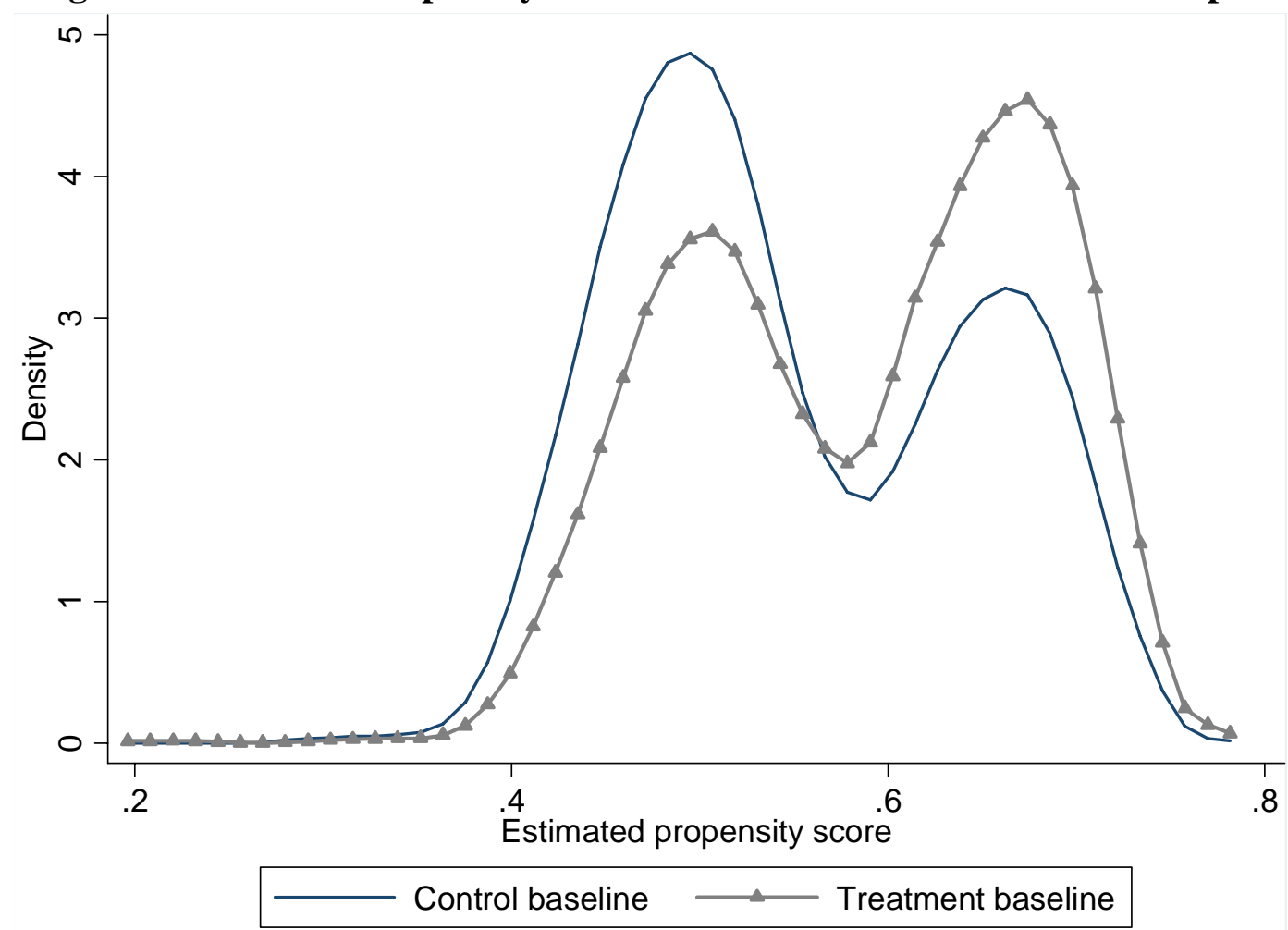

Table 1.- Field Operations First Phase, June 2010

\begin{tabular}{lcc}
\hline City & $\begin{array}{c}\text { Number of } \\
\text { households }\end{array}$ & $\begin{array}{c}\text { Number of persons 65 years } \\
\text { old or above }\end{array}$ \\
\hline Valladolid & 15,535 & 2,371 \\
Motul & 7,328 & 1,547 \\
Total & 22,863 & 3,918 \\
\hline
\end{tabular}

NOTE: The first phase of the research project is conducted in the cities of Valladolid and Motul. The household listing took place between June and July 2008, where individuals 65 years old or above were identified.

SOURCE: Aguila, Robles, and Vargas (2010). 
Table 2.- Descriptive Statistics Baseline First Phase

\begin{tabular}{|c|c|c|}
\hline Variable & $\begin{array}{c}\text { Treatment Town } \\
\text { (Valladolid) }\end{array}$ & $\begin{array}{c}\text { Control Town } \\
\text { (Motul) }\end{array}$ \\
\hline Mean age & 78.51 & 78.10 \\
\hline Standard .deviation. & (6.39) & $(6.71)$ \\
\hline Male (\%) & 43.05 & 48.01 \\
\hline \multicolumn{3}{|l|}{ Marital status (\%) } \\
\hline Single & 5.35 & 5.70 \\
\hline Couple & 53.32 & 54.48 \\
\hline Divorced/Separated & 3.36 & 2.68 \\
\hline Widowed & 37.88 & 37.14 \\
\hline Missing & 0.09 & 0.00 \\
\hline \multicolumn{3}{|l|}{ Education (\%) } \\
\hline No schooling & 42.11 & 29.57 \\
\hline Incomplete primary & 44.52 & 61.70 \\
\hline Primary or more & 12.08 & 8.38 \\
\hline Missing & 1.29 & 0.35 \\
\hline Speaks Maya (\%) & 76.70 & 79.28 \\
\hline Read and write a message in Spanish (\%) & 54.53 & 65.31 \\
\hline Living alone $(\%)$ & 12.47 & 13.50 \\
\hline Mean number of household residents & 2.54 & 2.58 \\
\hline Standard deviation & $(2.22)$ & $(2.21)$ \\
\hline No. Observations & 1,159 & 859 \\
\hline
\end{tabular}

NOTE: The first phase of the research project is conducted in the cities of Valladolid and Motul.

SOURCE: Baseline ENCAHEY first phase, 2008 
Table 3.- Difference-in-Differences of the Means

Variable (verbal scale [numeric codes])

\author{
Valladolid Valladolid Difference \\ Baseline follow-up Valladolid
}

Motul

Baseline
Motul

follow-
Difference Motul
Dif-in-dif of the means

Self-reported health (excellent, very good, good, fair, poor [1-

5])

Subjective mortality expectation (chances to live at least 10 years more [0-100])

Feel sad, blue or depressed for 2 weeks more during the past 3 months (yes-no[1-0])

Satisfied relation fam. members (very satisfied-very dissatisfied [1-5])

Satisfied hh income(very satisfied-very dissatisfied [15])

Satisfied social contacts (very satisfied-very dissatisfied [15])

Satisfied with job (very satisfied-very dissatisfied [15])

Satisfied with health (very satisfied-very dissatisfied [1-

$$
\text { 5]) }
$$

Satisfied life in general (very satisfied-very dissatisfied [1-

$$
\text { 5]) }
$$

Number of acute conditions

Often Pain (yes-no[1-0])

How strong is the pain (mild, moderate, severe $[1,2,3]$ )

$\begin{array}{ccccccc}3.96 & 3.84 & -0.117 & 4.05 & 3.85 & -0.204 & 0.0863 \\ (0.0211) & (0.0205) & (0.0294) & (0.0224) & (0.0225) & (0.0318) & (0.0367)^{*}\end{array}$

$\begin{array}{ccccccc}59.2 & 56.8 & -2.39 & 55.7 & 56.2 & 0.532 & 1.01 \\ (0.986) & (1.09) & (1.47) & (1.08) & (1.2) & (1.61) & (2.38)\end{array}$

$\begin{array}{ccccccc}0.598 & 0.44 & -0.158 & 0.511 & 0.376 & -0.135 & -0.0238 \\ (0.0157) & (0.0166) & (0.0228) & (0.0188) & (0.0183) & (0.0262) & (0.0332)\end{array}$

$\begin{array}{ccccccc}2.11 & 2.07 & -0.043 & 2.11 & 2.03 & -0.0865 & 0.0368 \\ (0.0217) & (0.0213) & (0.0304) & (0.0235) & (0.0226) & (0.0326) & (0.041)\end{array}$

$\begin{array}{ccccccc}2.58 & 2.33 & -0.247 & 2.6 & 2.4 & -0.198 & -0.0741 \\ (0.0268) & (0.0227) & (0.0352) & (0.0309) & (0.0282) & (0.0418) & (0.0523)\end{array}$

$\begin{array}{ccccccc}2.26 & 2.22 & -0.0424 & 2.25 & 2.17 & -0.0744 & 0.0118 \\ (0.0228) & (0.0207) & (0.0308) & (0.0246) & (0.0232) & (0.0338) & (0.0431)\end{array}$

$\begin{array}{ccccccc}2.39 & 2.28 & -0.108 & 2.34 & 2.23 & -0.111 & 0.00215 \\ (0.0254) & (0.0224) & (0.0339) & (0.0262) & (0.0257) & (0.0367) & (0.0442)\end{array}$

$\begin{array}{ccccccc}2.61 & 2.43 & -0.177 & 2.63 & 2.54 & -0.0881 & -0.0946 \\ (0.028) & (0.0251) & (0.0376) & (0.0311) & (0.031) & (0.0439) & (0.0502)\end{array}$

$\begin{array}{ccccccc}2.26 & 2.11 & -0.144 & 2.28 & 2.18 & -0.1 & -0.0409 \\ (0.0218) & (0.0159) & (0.027) & (0.0244) & (0.0228) & (0.0334) & (0.0408) \\ 0.178 & 0.119 & -0.0587 & 0.151 & 0.132 & -0.0198 & -0.0389 \\ (0.013) & (0.0105) & (0.0167) & (0.0134) & (0.0121) & (0.0181) & (0.0192)^{*} \\ 0.665 & 0.627 & -0.038 & 0.654 & 0.549 & -0.105 & 0.0668 \\ (0.0139) & (0.0142) & (0.0199) & (0.0163) & (0.017) & (0.0235) & (0.0263)^{*}\end{array}$

$\begin{array}{ccccccc}1.38 & 1.17 & -0.204 & 1.27 & 1.07 & -0.206 & 0.00253 \\ (0.034) & (0.0316) & (0.0464) & (0.0382) & (0.0376) & (0.0536) & (0.0595)\end{array}$




\begin{tabular}{|c|c|c|c|c|c|c|c|}
\hline Number of chronic conditions & $\begin{array}{c}0.908 \\
(0.029)\end{array}$ & $\begin{array}{c}0.876 \\
(0.0278)\end{array}$ & $\begin{array}{l}-0.0319 \\
(0.0402)\end{array}$ & $\begin{array}{c}1.03 \\
(0.0347)\end{array}$ & $\begin{array}{c}0.971 \\
(0.0326)\end{array}$ & $\begin{array}{c}-0.057 \\
(0.0476)\end{array}$ & $\begin{array}{c}0.0251 \\
(0.0442)\end{array}$ \\
\hline \multirow[t]{2}{*}{ Number of ADL's } & 4.6 & 4.07 & -0.529 & 4.75 & 4.17 & -0.586 & -0.177 \\
\hline & $(0.115)$ & $(0.108)$ & $(0.157)$ & $(0.13)$ & $(0.119)$ & $(0.176)$ & $(0.164)$ \\
\hline \multirow[t]{2}{*}{ Number of IADL's } & 0.76 & 0.63 & -0.129 & 0.653 & 0.524 & -0.129 & 0.00421 \\
\hline & $(0.0304)$ & $(0.0285)$ & $(0.0417)$ & $(0.0336)$ & $(0.0298)$ & $(0.0449)$ & $(0.0509)$ \\
\hline \multirow[t]{2}{*}{ Smoke now (yes-no [1-0]) } & 0.0311 & 0.0294 & -0.0017 & 0.0373 & 0.0315 & -0.00575 & 0.00411 \\
\hline & $(0.0051)$ & $(0.00496)$ & $(0.00711)$ & $(0.00647)$ & $(0.00597)$ & $(0.0088)$ & $(0.00571)$ \\
\hline \multirow[t]{2}{*}{ Number of cigarettes in a day } & 0.0811 & 0.0828 & 0.00173 & 0.192 & 0.129 & -0.0629 & 0.0646 \\
\hline & $(0.0205)$ & $(0.0219)$ & $(0.03)$ & $(0.0492)$ & $(0.0395)$ & $(0.0631)$ & $(0.0325)^{*}$ \\
\hline \multirow{3}{*}{$\begin{array}{l}\text { Drink alcoholic beverages } \\
\text { (yes-no[1-0]) }\end{array}$} & & & & & & & \\
\hline & 0.522 & 0.51 & -0.0116 & 0.591 & 0.74 & 0.149 & -0.161 \\
\hline & $(0.0147)$ & $(0.0147)$ & $(0.0208)$ & $(0.0168)$ & $(0.015)$ & $(0.0225)$ & $(0.0235)^{* *}$ \\
\hline \multirow{3}{*}{$\begin{array}{l}\text { Number of days a week drinks } \\
\text { alcoholic beverages }\end{array}$} & & & & & & & \\
\hline & 0.232 & 0.163 & -0.0689 & 0.214 & 0.216 & 0.00184 & -0.0516 \\
\hline & $(0.0216)$ & $(0.0192)$ & $(0.0289)$ & $(0.0268)$ & $(0.0258)$ & $(0.0372)$ & $(0.0358)$ \\
\hline \multirow[t]{2}{*}{ Number of drinks per day } & 0.345 & 0.287 & -0.0574 & 0.271 & 0.345 & 0.0745 & -0.138 \\
\hline & $(0.035)$ & $(0.0359)$ & $(0.0501)$ & $(0.0345)$ & $(0.0424)$ & $(0.0547)$ & $(0.0602)^{*}$ \\
\hline \multirow[t]{2}{*}{ Visited doctor (yes-no[1-0]) } & 0.414 & 0.522 & 0.109 & 0.489 & 0.509 & 0.0204 & 0.0882 \\
\hline & $(0.0145)$ & $(0.0147)$ & $(0.0206)$ & $(0.0171)$ & $(0.0171)$ & $(0.0241)$ & $(0.0274)^{* *}$ \\
\hline \multirow[t]{2}{*}{ Number of doctor visits } & 1.07 & 1.28 & 0.205 & 1.31 & 1.23 & -0.0805 & 0.286 \\
\hline & $(0.0518)$ & $(0.0582)$ & $(0.078)$ & $(0.0814)$ & $(0.0573)$ & $(0.0995)$ & $(0.112)^{*}$ \\
\hline \multirow[t]{2}{*}{$\begin{array}{l}\text { Visited a folk healer (yes- } \\
\text { no[1-0]) }\end{array}$} & 0.0501 & 0.0397 & -0.0104 & 0.0221 & 0.0291 & 0.00702 & -0.0174 \\
\hline & $(0.00641)$ & $(0.00574)$ & $(0.0086)$ & $(0.00502)$ & $(0.00575)$ & $(0.00763)$ & $(0.011)$ \\
\hline \multirow[t]{2}{*}{ Number of folk healer visits } & 0.108 & 0.0785 & -0.0295 & 0.0477 & 0.0606 & 0.0129 & -0.0439 \\
\hline & $(0.0166)$ & $(0.0146)$ & $(0.0222)$ & $(0.0145)$ & $(0.0162)$ & $(0.0217)$ & $(0.0297)$ \\
\hline \multirow[t]{2}{*}{ Visited a dentist (yes-no[1-0]) } & 0.0751 & 0.0664 & -0.00869 & 0.0653 & 0.0711 & 0.00583 & -0.0145 \\
\hline & $(0.00775)$ & $(0.00732)$ & $(0.0107)$ & $(0.00844)$ & $(0.00878)$ & $(0.0122)$ & $(0.0151)$ \\
\hline \multirow[t]{2}{*}{ Number of dentist visits } & 0.168 & 0.123 & -0.0453 & 0.126 & 0.129 & 0.0035 & -0.0502 \\
\hline & $(0.0241)$ & $(0.0171)$ & $(0.0296)$ & $(0.0199)$ & $(0.0202)$ & $(0.0284)$ & $(0.0389)$ \\
\hline \multirow{3}{*}{$\begin{array}{l}\text { Outpatient procedures (yes- } \\
\text { no[1-0]) }\end{array}$} & & & & & & & \\
\hline & 0.0112 & 0.0069 & -0.00433 & 0.0105 & 0.00932 & -0.00115 & -0.00316 \\
\hline & $(0.0031)$ & $(0.00243)$ & $(0.00394)$ & $(0.00348)$ & $(0.00328)$ & $(0.00478)$ & $(0.00623)$ \\
\hline \multicolumn{8}{|l|}{$\begin{array}{l}\text { Number of outpatient } \\
\text { procedures (ambulatory }\end{array}$} \\
\hline \multirow[t]{2}{*}{ surgery) } & 0.0199 & 0.0112 & -0.00866 & 0.0116 & 0.0105 & -0.00115 & -0.00748 \\
\hline & $(0.00705)$ & $(0.00447)$ & $(0.00835)$ & $(0.00402)$ & $(0.00385)$ & $(0.00556)$ & $(0.0101)$ \\
\hline \multirow[t]{2}{*}{$\begin{array}{l}\text { Consulted a pharmacist (yes- } \\
\text { no[1-0]) }\end{array}$} & 0.0771 & 0.0596 & -0.0175 & 0.0478 & 0.0315 & -0.0163 & -0.00101 \\
\hline & $(0.00786)$ & $(0.00696)$ & $(0.0105)$ & $(0.00729)$ & $(0.00596)$ & $(0.00942)$ & $(0.0135)$ \\
\hline \multirow{3}{*}{$\begin{array}{l}\text { Bought no medicines because } \\
\text { they are too expensive (yes- } \\
\text { no[1-0]) }\end{array}$} & & & & & & & \\
\hline & 0.238 & 0.125 & -0.113 & 0.186 & 0.133 & -0.0535 & -0.0595 \\
\hline & $(0.0125)$ & $(0.00973)$ & $(0.0159)$ & $(0.0133)$ & $(0.0116)$ & $(0.0177)$ & $(0.0219) * *$ \\
\hline
\end{tabular}


Pay out-of-pocket (oop) medical cost or medications

OP expenses paid by

relatives (yes-no[1-0])

OOP expenses paid by elderly eligible (yes-no[1-0])

Serious health problem but did not go to the doctor (yes-no[10])

Did not go to the doctor because of money (yes-no[10])

Relatives or friends pay your expenses (not true, sometimes true, often true, always true [14])

Feel a burden on your family or friends (not true, sometimes true, often true, always true [14])

Activities you used to do but can't do because of lack of money (yes-no[1-0])

Donation to the church but can't do due to money (yesno[1-0])

Donation to local parties but can't do due to money (yesno[1-0])

Donation to family or friends parties but can't do due to money (yes-no[1-0])

Community activities but can't do due to money (yes-no[1-0])

Help out family members but

$\begin{array}{ccccccc}0.459 & 0.436 & -0.0233 & 0.412 & 0.383 & -0.0294 & 0.00584 \\ (0.0147) & (0.0146) & (0.0207) & (0.0168) & (0.0166) & (0.0236) & (0.0284)\end{array}$

$\begin{array}{ccccccc}0.268 & 0.15 & -0.117 & 0.159 & 0.152 & -0.00682 & -0.111 \\ (0.013) & (0.0105) & (0.0167) & (0.0125) & (0.0123) & (0.0175) & (0.0219)^{* *}\end{array}$

$\begin{array}{ccccccc}0.168 & 0.239 & 0.0717 & 0.221 & 0.208 & -0.0137 & 0.0846 \\ (0.011) & (0.0125) & (0.0167) & (0.0142) & (0.0139) & (0.0198) & (0.0237)^{* *}\end{array}$

$\begin{array}{ccccccc}0.172 & 0.0814 & -0.0902 & 0.123 & 0.0606 & -0.0619 & -0.0285 \\ (0.0111) & (0.00805) & (0.0137) & (0.0112) & (0.00815) & (0.0139) & (0.0188)\end{array}$

$\begin{array}{ccccccc}0.104 & 0.0406 & -0.0638 & 0.0827 & 0.0349 & -0.0477 & -0.0161 \\ (0.00899) & (0.0058) & (0.0107) & (0.0094) & (0.00627) & (0.0113) & (0.0149)\end{array}$

$\begin{array}{ccccccc}2.19 & 2.19 & 0.000381 & 2.04 & 2.07 & 0.026 & -0.0214 \\ (0.0361) & (0.0358) & (0.0508) & (0.0406) & (0.0405) & (0.0574) & (0.0621)\end{array}$

$\begin{array}{ccccccc}1.92 & 2.07 & 0.154 & 2.04 & 1.89 & -0.154 & 0.573 \\ (0.0664) & (0.0727) & (0.0985) & (0.0775) & (0.0834) & (0.114) & (0.207)^{* *}\end{array}$

$\begin{array}{ccccccc}0.386 & 0.231 & -0.154 & 0.295 & 0.16 & -0.134 & -0.038 \\ (0.0156) & (0.0141) & (0.0211) & (0.0171) & (0.0139) & (0.0221) & (0.0312)\end{array}$

$\begin{array}{ccccccc}0.0224 & 0.0112 & -0.0112 & 0.0175 & 0.00698 & -0.0105 & -0.00074 \\ (0.00435) & (0.00309) & (0.00534) & (0.00447) & (0.00284) & (0.0053) & (0.00757)\end{array}$

$\begin{array}{ccccccc}0.00777 & 0.00173 & -0.00604 & 0.00466 & 0.00116 & -0.00349 & -0.00255 \\ (0.00258) & (0.00122) & (0.00285) & (0.00232) & (0.00116) & (0.0026) & (0.00386)\end{array}$

$\begin{array}{ccccccc}0.025 & 0.0112 & -0.0138 & 0.0268 & 0.00815 & -0.0186 & 0.00482 \\ (0.00459) & (0.00309) & (0.00554) & (0.00551) & (0.00307) & (0.00631) & (0.00845)\end{array}$

$\begin{array}{ccccccc}0.019 & 0.0104 & -0.00863 & 0.00582 & 0.00582 & 0 & -0.00863 \\ (0.00401) & (0.00297) & (0.00499) & (0.0026) & (0.0026) & (0.00367) & (0.00623)\end{array}$

$\begin{array}{llllllll}0.134 & 0.135 & 0.00173 & 0.118 & 0.0978 & -0.0198 & 0.0215\end{array}$


can’t do due to money (yesno[1-0])

Help out non-relatives but can't do due to money (yesno[1-0])

Travel to visit family or friends but can't do due to money (yes-no[1-0])

Sometimes we do not have enough to eat (yes-no[1-0])

Often we do not have enough to eat (yes-no[1-0])

Sometimes or often we don't have enough to eat because of money (yes-no[1-0])

Often worried run out of food last three months (never-

$$
\text { always [1-4])) }
$$

Often run out of food last three months (never-always [1-4]))

Skip or cut meals (never-

$$
\text { always [1-4]) }
$$

Often eat less than you felt you should (never-always [1-4])

Often hungry (never-always

$$
\text { [1-4]) }
$$

Not eat all day (never-always

$$
\text { [1-4]) }
$$

Food from charity (neveralways [1-4])

Spend on food at home last week

Spend on food away from home $\begin{array}{llllll}(0.01) & (0.0101) & (0.0142) & (0.011) & (0.0101) & (0.015)\end{array}$

$(0.02)$

$\begin{array}{ccccccc}0.0336 & 0.0121 & -0.0216 & 0.0198 & 0.00466 & -0.0151 & -0.00644 \\ (0.0053) & (0.00321) & (0.0062) & (0.00475) & (0.00232) & (0.00529) & (0.00811)\end{array}$

$\begin{array}{ccccccc}0.0595 & 0.0207 & -0.0388 & 0.0326 & 0.0116 & -0.021 & -0.0179 \\ (0.00695) & (0.00418) & (0.00812) & (0.00606) & (0.00366) & (0.00708) & (0.0107)\end{array}$

$\begin{array}{ccccccc}0.298 & 0.207 & -0.0906 & 0.352 & 0.282 & -0.0698 & -0.0207 \\ (0.0134) & (0.0119) & (0.018) & (0.0163) & (0.0154) & (0.0224) & (0.026)\end{array}$

$\begin{array}{lllllll}0.0544 & 0.0423 & -0.0121 & 0.071 & 0.0349 & -0.0361 & 0.024\end{array}$

$\begin{array}{lllllll}(0.00666) & (0.00591) & (0.00891) & (0.00877) & (0.00627) & (0.0108) & (0.0132)\end{array}$

$\begin{array}{ccccccc}0.267 & 0.247 & -0.0207 & 0.373 & 0.314 & -0.0582 & 0.0375 \\ (0.013) & (0.0127) & (0.0182) & (0.0165) & (0.0158) & (0.0229) & (0.0263)\end{array}$

$\begin{array}{ccccccc}1.93 & 1.78 & -0.142 & 1.91 & 1.66 & -0.245 & 0.0666 \\ (0.0282) & (0.0277) & (0.0395) & (0.0314) & (0.0298) & (0.0433) & (0.0532)\end{array}$

$\begin{array}{ccccccc}1.76 & 1.49 & -0.26 & 1.61 & 1.51 & -0.106 & -0.146 \\ (0.025) & (0.024) & (0.0346) & (0.0247) & (0.025) & (0.0351) & (0.046)^{* *}\end{array}$

$\begin{array}{ccccccc}1.66 & 1.42 & -0.244 & 1.59 & 1.43 & -0.157 & -0.0748 \\ (0.0242) & (0.0218) & (0.0325) & (0.0248) & (0.0238) & (0.0343) & (0.0446)\end{array}$

$\begin{array}{ccccccc}1.6 & 1.35 & -0.255 & 1.54 & 1.39 & -0.147 & -0.0995 \\ (0.0234) & (0.0201) & (0.0308) & (0.0231) & (0.0222) & (0.032) & (0.0422)^{*}\end{array}$

$\begin{array}{ccccccc}1.4 & 1.16 & -0.246 & 1.3 & 1.16 & -0.14 & -0.103 \\ (0.0205) & (0.0145) & (0.0251) & (0.0199) & (0.015) & (0.0249) & (0.0341)^{* *}\end{array}$

$\begin{array}{ccccccc}1.24 & 1.07 & -0.176 & 1.15 & 1.09 & -0.0591 & -0.121 \\ (0.0158) & (0.00943) & (0.0184) & (0.0136) & (0.0119) & (0.0181) & (0.0253)^{* *}\end{array}$

$\begin{array}{ccccccc}1.08 & 1.03 & -0.0491 & 1.04 & 1.05 & 0.00848 & -0.0565 \\ (0.0108) & (0.00691) & (0.0128) & (0.00779) & (0.00994) & (0.0126) & (0.018)^{* *}\end{array}$

$\begin{array}{lllllll}444 & 480 & 36.1 & 424 & 437 & 13.4 & 8.73\end{array}$

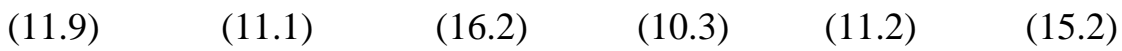

(20.6)

36.6
$-11.2$

22.9 


\begin{tabular}{|c|c|c|c|c|c|c|c|}
\hline \multirow{3}{*}{ Total Food } & $(6.69)$ & $(9.53)$ & $(11.6)$ & $(3.32)$ & (3.66) & $(4.94)$ & (13) \\
\hline & 485 & 516 & 31.2 & 446 & 469 & 22.8 & -15.1 \\
\hline & (17.5) & $(19.2)$ & (26) & (11.4) & $(12.6)$ & (17) & $(34.1)$ \\
\hline \multirow{3}{*}{$\begin{array}{l}\text { Received free food (yes-no [1- } \\
\text { 0]) }\end{array}$} & & & & & & & \\
\hline & 0.146 & 0.148 & 0.0016 & 0.0964 & 0.149 & 0.0529 & -0.0452 \\
\hline & $(0.0106)$ & $(0.0115)$ & $(0.0156)$ & $(0.0101)$ & $(0.0134)$ & $(0.0168)$ & $(0.0213)^{*}$ \\
\hline \multicolumn{8}{|l|}{$\begin{array}{l}\text { Eat diary products: milk, } \\
\text { cheese, yogurt (at least once a } \\
\text { day, at least once a week, } \\
\text { several times a month, once in }\end{array}$} \\
\hline & 2.84 & 2.68 & -0.166 & 3.03 & 2.91 & -0.119 & -0.0456 \\
\hline & $(0.0422)$ & $(0.0409)$ & $(0.0588)$ & $(0.0482)$ & $(0.0474)$ & $(0.0676)$ & $(0.0713)$ \\
\hline \\
\hline $\begin{array}{l}\text { least once a day, at least once a } \\
\text { week, several times a month, }\end{array}$ & & & & & & & \\
\hline \multirow[t]{2}{*}{ once in a while, never [1-5]) } & 2.07 & 2.15 & 0.0736 & 2.21 & 2.29 & 0.0767 & -0.00385 \\
\hline & $(0.0311)$ & $(0.0277)$ & $(0.0417)$ & $(0.0341)$ & $(0.0315)$ & $(0.0464)$ & $(0.0585)$ \\
\hline \multicolumn{8}{|l|}{$\begin{array}{l}\text { Eat meat, poultry or fish (at } \\
\text { least once a day, at least once a } \\
\text { week, several times a month, }\end{array}$} \\
\hline once in a while, never [1-5]) & 2.51 & 2.48 & -0.0281 & 2.51 & 2.52 & 0.0135 & -0.0432 \\
\hline & $(0.0322)$ & $(0.0284)$ & $(0.0429)$ & $(0.0355)$ & $(0.0336)$ & $(0.0489)$ & $(0.0601)$ \\
\hline \multicolumn{8}{|l|}{$\begin{array}{l}\text { Eat fruit or vegetables (at least } \\
\text { once a day, at least once a }\end{array}$} \\
\hline \multirow[t]{2}{*}{ once in a while, never [1-5]) } & 2.38 & 2.31 & -0.0779 & 2.37 & 2.33 & -0.0384 & -0.0412 \\
\hline & $(0.0355)$ & $(0.0316)$ & $(0.0475)$ & $(0.041)$ & $(0.0378)$ & $(0.0558)$ & $(0.0644)$ \\
\hline \multicolumn{8}{|l|}{$\begin{array}{l}\text { Eat tortillas, bread, crackers or } \\
\text { other cereals (at least once a } \\
\text { day, at least once a week, } \\
\text { several times a month. once in }\end{array}$} \\
\hline $\begin{array}{l}\text { several times a month, once in } \\
\text { a while, never [1-5]) }\end{array}$ & 1.12 & 1.09 & -0.0337 & 1.08 & 1.05 & -0.0231 & -0.0105 \\
\hline & $(0.0141)$ & $(0.0128)$ & $(0.0191)$ & $(0.0148)$ & $(0.0135)$ & $(0.02)$ & $(0.0267)$ \\
\hline \multicolumn{8}{|l|}{ Worked for pay, last month } \\
\hline (yes-no[1-0]) & $\begin{array}{c}0.165 \\
(0.0109)\end{array}$ & $\begin{array}{c}0.121 \\
(0.00959)\end{array}$ & $\begin{array}{l}-0.0439 \\
(0.0145)\end{array}$ & $\begin{array}{c}0.162 \\
(0.0126)\end{array}$ & $\begin{array}{c}0.164 \\
(0.0127)\end{array}$ & $\begin{array}{l}0.00233 \\
(0.0179)\end{array}$ & $\begin{array}{c}-0.0453 \\
(0.0155)^{* *}\end{array}$ \\
\hline \multicolumn{8}{|l|}{ Immediate recall (number of } \\
\hline & $(0.0511)$ & $(0.0547)$ & $(0.0749)$ & $(0.0608)$ & $(0.0604)$ & $(0.0857)$ & $(0.0834)^{* *}$ \\
\hline \multicolumn{8}{|l|}{ Delayed recall (number of } \\
\hline & $(0.0579)$ & $(0.0654)$ & $(0.0874)$ & $(0.0707)$ & $(0.0693)$ & $(0.099)$ & $(0.102)^{* *}$ \\
\hline \multicolumn{8}{|l|}{$\begin{array}{l}\text { Feel fear someone robbing you } \\
\text { (never, sometimes, usually, }\end{array}$} \\
\hline $\begin{array}{l}\text { (never, sometimes, usually, } \\
\text { always [1-4]) }\end{array}$ & 1.59 & 1.46 & -0.124 & 1.42 & 1.31 & -0.11 & -0.0363 \\
\hline & $(0.0291)$ & $(0.0265)$ & $(0.0393)$ & $(0.0287)$ & $(0.0255)$ & $(0.0384)$ & $(0.0531)$ \\
\hline $\begin{array}{l}\text { Feel fear someone close to you } \\
\text { will take your money (never, }\end{array}$ & 1.24 & 1.21 & -0.035 & 1.18 & 1.14 & -0.0429 & 0.0129 \\
\hline
\end{tabular}


sometimes, usually, always [1-

4])

Money in a safe place (yes-no

$\begin{array}{llllll}(0.0198) & (0.0178) & (0.0266) & (0.0181) & (0.0184) & (0.0258)\end{array}$

[1-0])

$\begin{array}{ccccccc}0.0301 & 0.0212 & -0.00885 & 0.0155 & 0.0114 & -0.00405 & -0.00355 \\ (0.0055) & (0.00482) & (0.00732) & (0.00464) & (0.00403) & (0.00614) & (0.01)\end{array}$

Feel verbally or physically abused (never, sometimes, usually, always [1-4])

$\begin{array}{ccccccc}1.13 & 1.11 & -0.0167 & 1.1 & 1.1 & -0.00098 & -0.0182 \\ (0.0136) & (0.0137) & (0.0193) & (0.014) & (0.0163) & (0.0215) & (0.0287)\end{array}$

NOTE: Scales of variables shown in the first column. SOURCE: Baseline and follow-up ENCAHEY, 2008 and 2009 $* *=$ significant at $5 \%$ level of confidence. $*=$ significant at $10 \%$ level of confidence. 
Table 4a.- Treatment Regressions

\begin{tabular}{|c|c|c|c|c|c|c|c|c|}
\hline & $\begin{array}{l}\text { Self-reported } \\
\text { health } \\
\text { (excellent, } \\
\text { very good, } \\
\text { good, fair, } \\
\text { poor [1-5]) }\end{array}$ & $\begin{array}{l}\text { Subjective } \\
\text { mortality } \\
\text { expectation } \\
\text { (chances to } \\
\text { leave at least } \\
10 \text { years } \\
\text { more [1-100 }\end{array}$ & $\begin{array}{l}\text { Feel sad, } \\
\text { blue or } \\
\text { depressed } \\
\text { for } 2 \text { weeks } \\
\text { or more } \\
\text { during the } \\
\text { last } 3 \\
\text { months (yes- } \\
\text { no }\end{array}$ & $\begin{array}{l}\text { Satisfied } \\
\text { relation fam. } \\
\text { members } \\
\text { (very } \\
\text { satisfied- } \\
\text { very } \\
\text { unsatisfied } \\
{[1-5] \text { ) }}\end{array}$ & $\begin{array}{l}\text { Satisfied hh } \\
\text { income (very } \\
\text { satisfied- } \\
\text { very } \\
\text { unsatisfied } \\
{[1-5] \text { ) }}\end{array}$ & $\begin{array}{l}\text { Satisfied } \\
\text { social } \\
\text { contacts } \\
\text { (very } \\
\text { satisfied- } \\
\text { very } \\
\text { unsatisfied } \\
\text { [1-5]) }\end{array}$ & $\begin{array}{l}\text { Satisfied } \\
\text { with job } \\
\text { (very } \\
\text { satisfied- } \\
\text { very } \\
\text { unsatisfied } \\
\text { [1-5]) }\end{array}$ & $\begin{array}{l}\text { Satisfied } \\
\text { with health } \\
\text { (very } \\
\text { satisfied- } \\
\text { very } \\
\text { unsatisfied } \\
\text { [1-5]) }\end{array}$ \\
\hline Wave & $\begin{array}{l}-0.229 \\
(0.045) * *\end{array}$ & $\begin{array}{l}0.401 \\
(2.401)\end{array}$ & $\begin{array}{l}-0.203 \\
(0.037)^{* *}\end{array}$ & $\begin{array}{l}-0.100 \\
(0.046)^{*}\end{array}$ & $\begin{array}{l}-0.189 \\
(0.060) * *\end{array}$ & $\begin{array}{l}-0.065 \\
(0.050)\end{array}$ & $\begin{array}{l}-0.145 \\
(0.052) * *\end{array}$ & $\begin{array}{l}-0.061 \\
(0.061)\end{array}$ \\
\hline Valladolid & $\begin{array}{l}0.089 \\
(0.032) * *\end{array}$ & $\begin{array}{l}-4.302 \\
(1.575)^{* *}\end{array}$ & $\begin{array}{l}-0.076 \\
(0.025) * *\end{array}$ & $\begin{array}{l}-0.004 \\
(0.034)\end{array}$ & $\begin{array}{l}0.009 \\
(0.044)\end{array}$ & $\begin{array}{l}-0.015 \\
(0.035)\end{array}$ & $\begin{array}{l}-0.029 \\
(0.038)\end{array}$ & $\begin{array}{l}0.013 \\
(0.044)\end{array}$ \\
\hline Valladolid wave 2 & $\begin{array}{l}0.085 \\
(0.037)^{*}\end{array}$ & $\begin{array}{l}-3.107 \\
(2.219)\end{array}$ & $\begin{array}{l}-0.018 \\
(0.034)\end{array}$ & $\begin{array}{l}0.046 \\
(0.041)\end{array}$ & $\begin{array}{l}-0.049 \\
(0.053)\end{array}$ & $\begin{array}{l}0.031 \\
(0.044)\end{array}$ & $\begin{array}{l}0.021 \\
(0.045)\end{array}$ & $\begin{array}{l}-0.092 \\
(0.051)\end{array}$ \\
\hline Age & $\begin{array}{l}0.049 \\
(0.037)\end{array}$ & $\begin{array}{l}-5.887 \\
(1.973)^{* *}\end{array}$ & $\begin{array}{l}0.037 \\
(0.031)\end{array}$ & $\begin{array}{l}-0.026 \\
(0.049)\end{array}$ & $\begin{array}{l}-0.080 \\
(0.046)\end{array}$ & $\begin{array}{l}0.014 \\
(0.042)\end{array}$ & $\begin{array}{l}-0.009 \\
(0.052)\end{array}$ & $\begin{array}{l}-0.041 \\
(0.058)\end{array}$ \\
\hline Age squared & $\begin{array}{l}-0.000 \\
(0.000)\end{array}$ & $\begin{array}{l}0.036 \\
(0.012) * *\end{array}$ & $\begin{array}{l}-0.000 \\
(0.000)\end{array}$ & $\begin{array}{l}0.000 \\
(0.000)\end{array}$ & $\begin{array}{l}0.000 \\
(0.000)\end{array}$ & $\begin{array}{l}-0.000 \\
(0.000)\end{array}$ & $\begin{array}{l}0.000 \\
(0.000)\end{array}$ & $\begin{array}{l}0.000 \\
(0.000)\end{array}$ \\
\hline Gender (male=1) & $\begin{array}{l}-0.088 \\
(0.025) * *\end{array}$ & $\begin{array}{l}3.756 \\
(1.111)^{* *}\end{array}$ & $\begin{array}{l}-0.135 \\
(0.019) * *\end{array}$ & $\begin{array}{l}0.022 \\
(0.026)\end{array}$ & $\begin{array}{l}0.035 \\
(0.029)\end{array}$ & $\begin{array}{l}-0.044 \\
(0.026)\end{array}$ & $\begin{array}{l}0.022 \\
(0.029)\end{array}$ & $\begin{array}{l}-0.109 \\
(0.033) * *\end{array}$ \\
\hline Speaks Maya & $\begin{array}{l}-0.035 \\
(0.039)\end{array}$ & $\begin{array}{l}0.850 \\
(1.746)\end{array}$ & $\begin{array}{l}-0.040 \\
(0.028)\end{array}$ & $\begin{array}{l}0.034 \\
(0.039)\end{array}$ & $\begin{array}{l}0.019 \\
(0.050)\end{array}$ & $\begin{array}{l}0.070 \\
(0.038)\end{array}$ & $\begin{array}{l}0.014 \\
(0.047)\end{array}$ & $\begin{array}{l}0.038 \\
(0.048)\end{array}$ \\
\hline $\begin{array}{l}\text { Reads/writes } \\
\text { Spanish }\end{array}$ & 0.007 & -1.714 & -0.050 & -0.063 & -0.012 & -0.063 & -0.053 & -0.007 \\
\hline & $(0.034)$ & (1.759) & $(0.027)$ & $(0.036)$ & $(0.048)$ & $(0.039)$ & $(0.041)$ & $(0.046)$ \\
\hline Lives alone & $\begin{array}{l}-0.046 \\
(0.045)\end{array}$ & $\begin{array}{l}1.122 \\
(2.072)\end{array}$ & $\begin{array}{l}0.031 \\
(0.033)\end{array}$ & $\begin{array}{l}0.113 \\
(0.050)^{*}\end{array}$ & $\begin{array}{l}0.104 \\
(0.055)\end{array}$ & $\begin{array}{l}0.045 \\
(0.047)\end{array}$ & $\begin{array}{l}0.046 \\
(0.054)\end{array}$ & $\begin{array}{l}-0.059 \\
(0.057)\end{array}$ \\
\hline household size & $\begin{array}{l}-0.003 \\
(0.006)\end{array}$ & $\begin{array}{l}-0.265 \\
(0.279)\end{array}$ & $\begin{array}{l}0.001 \\
(0.005)\end{array}$ & $\begin{array}{l}0.000 \\
(0.006)\end{array}$ & $\begin{array}{l}0.008 \\
(0.008)\end{array}$ & $\begin{array}{l}0.017 \\
(0.007)^{*}\end{array}$ & $\begin{array}{l}0.010 \\
(0.008)\end{array}$ & $\begin{array}{l}0.000 \\
(0.009)\end{array}$ \\
\hline Incomplete primary & $\begin{array}{l}0.003 \\
(0.028)\end{array}$ & $\begin{array}{l}1.432 \\
(1.339)\end{array}$ & $\begin{array}{l}0.018 \\
(0.021)\end{array}$ & $\begin{array}{l}-0.051 \\
(0.028)\end{array}$ & $\begin{array}{l}-0.027 \\
(0.036)\end{array}$ & $\begin{array}{l}-0.004 \\
(0.031)\end{array}$ & $\begin{array}{l}-0.076 \\
(0.033)^{*}\end{array}$ & $\begin{array}{l}0.087 \\
(0.037)^{*}\end{array}$ \\
\hline Primary & -0.142 & 0.987 & -0.062 & -0.324 & -0.087 & -0.218 & -0.210 & -0.027 \\
\hline
\end{tabular}




\begin{tabular}{lllllllll}
\hline & $(0.046)^{* *}$ & $(1.926)$ & $(0.033)$ & $(0.046)^{* *}$ & $(0.053)$ & $(0.044)^{* *}$ & $(0.050)^{* *}$ & $(0.056)$ \\
Couple & 0.107 & 4.545 & 0.085 & -0.041 & 0.074 & 0.009 & 0.019 \\
& $(0.070)$ & $(2.644)$ & $(0.047)$ & $(0.054)$ & $(0.064)$ & $(0.047)$ & $(0.056)$ & $(0.029$ \\
Divorced/Separated & -0.057 & 1.146 & 0.078 & 0.096 & 0.131 & 0.033 & -0.023 & 0.126 \\
& $(0.107)$ & $(3.914)$ & $(0.066)$ & $(0.101)$ & $(0.092)$ & $(0.080)$ & $(0.090)$ & $(0.115)$ \\
Widow & 0.079 & 1.233 & 0.108 & -0.043 & 0.088 & 0.015 & 0.053 & 0.006 \\
& $(0.071)$ & $(2.704)$ & $(0.047)^{*}$ & $(0.056)$ & $(0.063)$ & $(0.048)$ & $(0.056)$ & $(0.075)$ \\
Constant & 1.798 & 299.185 & -0.893 & 3.231 & 5.644 & 1.599 & 2.463 & 4.016 \\
& $(1.499)$ & $(79.642)^{* *}$ & $(1.238)$ & $(1.947)$ & $(1.867)^{* *}$ & $(1.688)$ & $(2.078)$ & $(2.329)$ \\
Observations & 4007 & 2405 & 3271 & 3258 & 3253 & 3248 & 3257 & 3261 \\
R-squared & 0.03 & 0.02 & 0.06 & 0.04 & 0.03 & 0.02 & 0.03 & 0.02 \\
F-test education & 5.77 & 0.57 & 3.62 & 24.95 & 1.35 & 15.69 & 9.11 & 4.15 \\
Prob>F & 0.003 & 0.565 & 0.027 & 0.845 & 0.215 & 0.000 & 0.005 & 0.016 \\
F-test age & 1.827 & 5.093 & 2.251 & 0.169 & 1.538 & 0.956 & 5.251 & 1.905 \\
Prob>F & 0.161 & 0.006 & 0.106 & 0.000 & 0.261 & 0.385 & 0.000 & 0.149 \\
age parabola & 86.322 & 82.265 & 87.088 & 78.595 & 80.519 & 103.706 & 41.510 & 70.954 \\
min/max & & & & & & & &
\end{tabular}

NOTE: The reference categories are wave 1, Motul, female, do not speak Maya, do not read write a message in Spanish, no schooling, and single. SOURCE: Baseline and first follow-up ENCAHEY, 2008 and 2009.

** = significant at $5 \%$ level of confidence. $*$ = significant at $10 \%$ level of confidence 
Table 4b.- Treatment Regressions

\begin{tabular}{|c|c|c|c|c|c|c|c|c|}
\hline & $\begin{array}{l}\text { Satisfied life } \\
\text { in general } \\
\text { (very } \\
\text { satisfied-very } \\
\text { unsatisfied [1- } \\
\text { 5]) }\end{array}$ & $\begin{array}{l}\text { Number of } \\
\text { acute } \\
\text { conditions }\end{array}$ & $\begin{array}{l}\text { Often pain } \\
\text { (yes-no [1- } \\
0] \text { ) }\end{array}$ & $\begin{array}{l}\text { How strong } \\
\text { is the pain } \\
\text { (mild, } \\
\text { moderate, } \\
\text { severe [1- } \\
3] \text { ) }\end{array}$ & $\begin{array}{l}\text { Number of } \\
\text { chronic } \\
\text { conditions }\end{array}$ & $\begin{array}{l}\text { Number of } \\
\text { ADL's }\end{array}$ & $\begin{array}{l}\text { Number of } \\
\text { IADL's }\end{array}$ & $\begin{array}{l}\text { Smokes } \\
\text { now (yes- } \\
\text { no [1-0]) }\end{array}$ \\
\hline Wave & $\begin{array}{l}-0.089 \\
(0.049)\end{array}$ & $\begin{array}{l}-0.032 \\
(0.026)\end{array}$ & $\begin{array}{l}-0.120 \\
(0.030)^{* *}\end{array}$ & $\begin{array}{l}-0.278 \\
(0.073) * *\end{array}$ & $\begin{array}{l}-0.055 \\
(0.061)\end{array}$ & $\begin{array}{l}-1.596 \\
(0.235) * *\end{array}$ & $\begin{array}{l}-0.288 \\
(0.066)^{* *}\end{array}$ & $\begin{array}{l}-0.007 \\
(0.009)\end{array}$ \\
\hline Valladolid & $\begin{array}{l}0.018 \\
(0.035)\end{array}$ & $\begin{array}{l}-0.018 \\
(0.019)\end{array}$ & $\begin{array}{l}-0.007 \\
(0.022)\end{array}$ & $\begin{array}{l}-0.081 \\
(0.054)\end{array}$ & $\begin{array}{l}0.139 \\
(0.046) * *\end{array}$ & $\begin{array}{l}0.284 \\
(0.173)\end{array}$ & $\begin{array}{l}-0.071 \\
(0.047)\end{array}$ & $\begin{array}{l}0.003 \\
(0.008)\end{array}$ \\
\hline Valladolid wave 2 & $\begin{array}{l}-0.044 \\
(0.041)\end{array}$ & $\begin{array}{l}-0.035 \\
(0.020)\end{array}$ & $\begin{array}{l}0.070 \\
(0.027)^{*}\end{array}$ & $\begin{array}{l}0.012 \\
(0.061)\end{array}$ & $\begin{array}{l}0.028 \\
(0.046)\end{array}$ & $\begin{array}{l}0.139 \\
(0.171)\end{array}$ & $\begin{array}{l}0.018 \\
(0.052)\end{array}$ & $\begin{array}{l}0.004 \\
(0.006)\end{array}$ \\
\hline Age & $\begin{array}{l}0.024 \\
(0.038)\end{array}$ & $\begin{array}{l}0.050 \\
(0.016)^{* *}\end{array}$ & $\begin{array}{l}0.032 \\
(0.025)\end{array}$ & $\begin{array}{l}0.099 \\
(0.055)\end{array}$ & $\begin{array}{l}0.192 \\
(0.047) * *\end{array}$ & $\begin{array}{l}0.395 \\
(0.216)\end{array}$ & $\begin{array}{l}0.043 \\
(0.058)\end{array}$ & $\begin{array}{l}-0.008 \\
(0.009)\end{array}$ \\
\hline Age squared & $\begin{array}{l}-0.000 \\
(0.000)\end{array}$ & $\begin{array}{l}-0.000 \\
(0.000)^{* *}\end{array}$ & $\begin{array}{l}-0.000 \\
(0.000)\end{array}$ & $\begin{array}{l}-0.001 \\
(0.000)\end{array}$ & $\begin{array}{l}-0.001 \\
(0.000) * *\end{array}$ & $\begin{array}{l}-0.002 \\
(0.001)\end{array}$ & $\begin{array}{l}-0.000 \\
(0.000)\end{array}$ & $\begin{array}{l}0.000 \\
(0.000)\end{array}$ \\
\hline Gender (male=1) & $\begin{array}{l}-0.030 \\
(0.024)\end{array}$ & $\begin{array}{l}-0.002 \\
(0.014)\end{array}$ & $\begin{array}{l}-0.037 \\
(0.018)^{*}\end{array}$ & $\begin{array}{l}-0.108 \\
(0.042)^{*}\end{array}$ & $\begin{array}{l}-0.260 \\
(0.039) * *\end{array}$ & $\begin{array}{l}-0.793 \\
(0.144) * *\end{array}$ & $\begin{array}{l}-0.180 \\
(0.035)^{* *}\end{array}$ & $\begin{array}{l}0.051 \\
(0.007)^{* *}\end{array}$ \\
\hline Speaks Maya & $\begin{array}{l}0.066 \\
(0.037)\end{array}$ & $\begin{array}{l}-0.015 \\
(0.022)\end{array}$ & $\begin{array}{l}-0.008 \\
(0.025)\end{array}$ & $\begin{array}{l}-0.042 \\
(0.060)\end{array}$ & $\begin{array}{l}0.032 \\
(0.053)\end{array}$ & $\begin{array}{l}-0.593 \\
(0.210) * *\end{array}$ & $\begin{array}{l}-0.110 \\
(0.056)^{*}\end{array}$ & $\begin{array}{l}-0.009 \\
(0.010)\end{array}$ \\
\hline Reads/writes Spanish & $\begin{array}{l}-0.061 \\
(0.040)\end{array}$ & $\begin{array}{l}0.003 \\
(0.021)\end{array}$ & $\begin{array}{l}-0.011 \\
(0.023)\end{array}$ & $\begin{array}{l}-0.054 \\
(0.055)\end{array}$ & $\begin{array}{l}-0.048 \\
(0.047)\end{array}$ & $\begin{array}{l}-0.701 \\
(0.177)^{* *}\end{array}$ & $\begin{array}{l}-0.080 \\
(0.050)\end{array}$ & $\begin{array}{l}0.005 \\
(0.007)\end{array}$ \\
\hline Lives alone & $\begin{array}{l}0.052 \\
(0.043)\end{array}$ & $\begin{array}{l}-0.028 \\
(0.025)\end{array}$ & $\begin{array}{l}0.001 \\
(0.031)\end{array}$ & $\begin{array}{l}-0.058 \\
(0.071)\end{array}$ & $\begin{array}{l}-0.064 \\
(0.064)\end{array}$ & $\begin{array}{l}-0.099 \\
(0.245)\end{array}$ & $\begin{array}{l}0.006 \\
(0.062)\end{array}$ & $\begin{array}{l}0.003 \\
(0.012)\end{array}$ \\
\hline household size & $\begin{array}{l}0.000 \\
(0.006)\end{array}$ & $\begin{array}{l}0.005 \\
(0.004)\end{array}$ & $\begin{array}{l}-0.000 \\
(0.004)\end{array}$ & $\begin{array}{l}-0.005 \\
(0.010)\end{array}$ & $\begin{array}{l}-0.009 \\
(0.009)\end{array}$ & $\begin{array}{l}-0.018 \\
(0.035)\end{array}$ & $\begin{array}{l}-0.003 \\
(0.009)\end{array}$ & $\begin{array}{l}-0.002 \\
(0.002)\end{array}$ \\
\hline Incomplete primary & $\begin{array}{l}0.006 \\
(0.028)\end{array}$ & $\begin{array}{l}0.024 \\
(0.017)\end{array}$ & $\begin{array}{l}-0.028 \\
(0.019)\end{array}$ & $\begin{array}{l}-0.092 \\
(0.044) *\end{array}$ & $\begin{array}{l}0.058 \\
(0.041)\end{array}$ & $\begin{array}{l}0.320 \\
(0.147) *\end{array}$ & $\begin{array}{l}-0.021 \\
(0.039)\end{array}$ & $\begin{array}{l}0.002 \\
(0.007)\end{array}$ \\
\hline Primary & $\begin{array}{l}-0.157 \\
(0.041)^{* *}\end{array}$ & $\begin{array}{l}0.102 \\
(0.028)^{* *}\end{array}$ & $\begin{array}{l}-0.152 \\
(0.031)^{* *}\end{array}$ & $\begin{array}{l}-0.276 \\
(0.072)^{* *}\end{array}$ & $\begin{array}{l}0.009 \\
(0.065)\end{array}$ & $\begin{array}{l}-0.339 \\
(0.223)\end{array}$ & $\begin{array}{l}-0.129 \\
(0.056)^{*}\end{array}$ & $\begin{array}{l}0.022 \\
(0.015)\end{array}$ \\
\hline Couple & $\begin{array}{l}0.043 \\
(0.050)\end{array}$ & $\begin{array}{l}0.052 \\
(0.023)^{*}\end{array}$ & $\begin{array}{l}0.109 \\
(0.043) *\end{array}$ & $\begin{array}{l}0.289 \\
(0.091)^{* *}\end{array}$ & $\begin{array}{l}0.151 \\
(0.081)\end{array}$ & $\begin{array}{l}0.176 \\
(0.331)\end{array}$ & $\begin{array}{l}-0.027 \\
(0.085)\end{array}$ & $\begin{array}{l}0.015 \\
(0.013)\end{array}$ \\
\hline Divorced/Separated & $\begin{array}{l}0.036 \\
(0.073)\end{array}$ & $\begin{array}{l}0.144 \\
(0.052)^{* *}\end{array}$ & $\begin{array}{l}0.131 \\
(0.059)^{*}\end{array}$ & $\begin{array}{l}0.378 \\
(0.137) * *\end{array}$ & $\begin{array}{l}0.283 \\
(0.130)^{*}\end{array}$ & $\begin{array}{l}0.218 \\
(0.457)\end{array}$ & $\begin{array}{l}-0.165 \\
(0.111)\end{array}$ & $\begin{array}{l}-0.011 \\
(0.014)\end{array}$ \\
\hline
\end{tabular}




\begin{tabular}{lllllllll} 
Widow & 0.032 & 0.076 & 0.063 & 0.197 & 0.103 & 0.394 & 0.001 & 0.011 \\
& $(0.050)$ & $(0.024)^{* *}$ & $(0.042)$ & $(0.089)^{*}$ & $(0.081)$ & $(0.331)$ & $(0.085)$ & $(0.012)$ \\
Constant & 1.230 & -1.919 & -0.743 & -2.745 & -6.663 & -14.344 & -1.595 & 0.337 \\
& $(1.547)$ & $(0.683)^{* *}$ & $(1.007)$ & $(2.251)$ & $(1.944)^{* *}$ & $(8.720)$ & $(2.346)$ & $(0.366)$ \\
Observations & 3257 & 4011 & 4006 & 4003 & 4011 & 3769 & 4002 & 4008 \\
R-squared & 0.02 & 0.02 & 0.02 & 0.03 & 0.03 & 0.07 & 0.05 & 0.03 \\
F-test education & 10.30 & 6.71 & 11.95 & 7.45 & 1.15 & 6.07 & 2.91 & 1.06 \\
Prob>F & 0.000 & 0.005 & 0.174 & 0.108 & 0.318 & 0.000 & 0.055 & 0.347 \\
F-test age & 0.292 & 5.244 & 1.750 & 2.227 & 20.122 & 36.032 & 35.543 & 0.910 \\
Prob>F & 0.747 & 0.001 & 0.000 & 0.001 & 0.000 & 0.002 & 0.000 & 0.403 \\
age parabola min/max & 84.360 & 81.676 & 86.865 & 85.204 & 77.917 & 107.069 & 184.752 & 89.213 \\
\hline
\end{tabular}

NOTE: The reference categories are wave 1, Motul, female, do not speak Maya, do not read write a message in Spanish, no schooling, and single. SOURCE: Baseline and first follow-up ENCAHEY, 2008 and 2009.

$* *=$ significant at $5 \%$ level of confidence. ${ }^{*}=$ significant at $10 \%$ level of confidence 
Table 4c.- Treatment Regressions

\begin{tabular}{|c|c|c|c|c|c|c|c|c|}
\hline & $\begin{array}{l}\text { Number of } \\
\text { cigarettes in a } \\
\text { day }\end{array}$ & $\begin{array}{l}\text { Drink } \\
\text { alcoholic } \\
\text { beverages } \\
\text { (yes-no [1- } \\
0] \text { ) }\end{array}$ & $\begin{array}{l}\text { Number of } \\
\text { days a week } \\
\text { drinks } \\
\text { alcoholic } \\
\text { beverages }\end{array}$ & $\begin{array}{l}\text { Number of } \\
\text { drinks per } \\
\text { day }\end{array}$ & $\begin{array}{l}\text { Visited a } \\
\text { doctor (yes- } \\
\text { no }[1-0])\end{array}$ & $\begin{array}{l}\text { Number of } \\
\text { doctor visits }\end{array}$ & $\begin{array}{l}\text { Visited a } \\
\text { folk healer } \\
\text { (yes-no [1- } \\
0] \text { ) }\end{array}$ & $\begin{array}{l}\text { Number of } \\
\text { folk healer } \\
\text { visits }\end{array}$ \\
\hline Wave & $\begin{array}{l}-0.117 \\
(0.060)\end{array}$ & $\begin{array}{l}0.115 \\
(0.030) * *\end{array}$ & $\begin{array}{l}0.033 \\
(0.055)\end{array}$ & $\begin{array}{l}0.116 \\
(0.088)\end{array}$ & $\begin{array}{l}0.087 \\
(0.034)^{*}\end{array}$ & $\begin{array}{l}0.129 \\
(0.117)\end{array}$ & $\begin{array}{l}-0.025 \\
(0.014)\end{array}$ & $\begin{array}{l}-0.034 \\
(0.033)\end{array}$ \\
\hline Valladolid & $\begin{array}{l}0.113 \\
(0.057)^{*}\end{array}$ & $\begin{array}{l}0.040 \\
(0.022)\end{array}$ & $\begin{array}{l}-0.037 \\
(0.036)\end{array}$ & $\begin{array}{l}-0.114 \\
(0.051)^{*}\end{array}$ & $\begin{array}{l}0.072 \\
(0.024)^{* *}\end{array}$ & $\begin{array}{l}0.248 \\
(0.100)^{*}\end{array}$ & $\begin{array}{l}-0.024 \\
(0.008) * *\end{array}$ & $\begin{array}{l}-0.053 \\
(0.024)^{*}\end{array}$ \\
\hline Valladolid wave 2 & $\begin{array}{l}0.068 \\
(0.035)\end{array}$ & $\begin{array}{l}-0.161 \\
(0.025) * *\end{array}$ & $\begin{array}{l}-0.075 \\
(0.042)\end{array}$ & $\begin{array}{l}-0.135 \\
(0.063)^{*}\end{array}$ & $\begin{array}{l}0.083 \\
(0.029)^{* *}\end{array}$ & $\begin{array}{l}0.274 \\
(0.115)^{*}\end{array}$ & $\begin{array}{l}-0.013 \\
(0.011)\end{array}$ & $\begin{array}{l}-0.034 \\
(0.030)\end{array}$ \\
\hline Age & $\begin{array}{l}0.027 \\
(0.038)\end{array}$ & $\begin{array}{l}-0.007 \\
(0.023)\end{array}$ & $\begin{array}{l}-0.047 \\
(0.032)\end{array}$ & $\begin{array}{l}-0.089 \\
(0.054)\end{array}$ & $\begin{array}{l}0.014 \\
(0.030)\end{array}$ & $\begin{array}{l}0.131 \\
(0.096)\end{array}$ & $\begin{array}{l}0.010 \\
(0.007)\end{array}$ & $\begin{array}{l}0.042 \\
(0.018)^{*}\end{array}$ \\
\hline Age squared & $\begin{array}{l}-0.000 \\
(0.000)\end{array}$ & $\begin{array}{l}0.000 \\
(0.000)\end{array}$ & $\begin{array}{l}0.000 \\
(0.000)\end{array}$ & $\begin{array}{l}0.000 \\
(0.000)\end{array}$ & $\begin{array}{l}-0.000 \\
(0.000)\end{array}$ & $\begin{array}{l}-0.001 \\
(0.001)\end{array}$ & $\begin{array}{l}-0.000 \\
(0.000)\end{array}$ & $\begin{array}{l}-0.000 \\
(0.000)^{*}\end{array}$ \\
\hline Gender (male=1) & $\begin{array}{l}0.185 \\
(0.038) * *\end{array}$ & $\begin{array}{l}0.361 \\
(0.016) * *\end{array}$ & $\begin{array}{l}0.144 \\
(0.024) * *\end{array}$ & $\begin{array}{l}0.283 \\
(0.039) * *\end{array}$ & $\begin{array}{l}-0.078 \\
(0.018)^{* *}\end{array}$ & $\begin{array}{l}-0.268 \\
(0.069) * *\end{array}$ & $\begin{array}{l}0.019 \\
(0.006)^{* *}\end{array}$ & $\begin{array}{l}0.031 \\
(0.017)\end{array}$ \\
\hline Speaks Maya & $\begin{array}{l}-0.082 \\
(0.068)\end{array}$ & $\begin{array}{l}-0.081 \\
(0.025) * *\end{array}$ & $\begin{array}{l}-0.040 \\
(0.044)\end{array}$ & $\begin{array}{l}-0.024 \\
(0.061)\end{array}$ & $\begin{array}{l}0.049 \\
(0.027)\end{array}$ & $\begin{array}{l}0.191 \\
(0.099)\end{array}$ & $\begin{array}{l}-0.020 \\
(0.012)\end{array}$ & $\begin{array}{l}-0.014 \\
(0.025)\end{array}$ \\
\hline Reads/writes Spanish & $\begin{array}{l}0.009 \\
(0.030)\end{array}$ & $\begin{array}{l}0.041 \\
(0.023)\end{array}$ & $\begin{array}{l}0.081 \\
(0.033)^{*}\end{array}$ & $\begin{array}{l}0.061 \\
(0.055)\end{array}$ & $\begin{array}{l}0.036 \\
(0.024)\end{array}$ & $\begin{array}{l}0.070 \\
(0.094)\end{array}$ & $\begin{array}{l}-0.024 \\
(0.010)^{*}\end{array}$ & $\begin{array}{l}-0.053 \\
(0.027)\end{array}$ \\
\hline Lives alone & $\begin{array}{l}0.079 \\
(0.070)\end{array}$ & $\begin{array}{l}0.022 \\
(0.030)\end{array}$ & $\begin{array}{l}-0.022 \\
(0.044)\end{array}$ & $\begin{array}{l}0.052 \\
(0.070)\end{array}$ & $\begin{array}{l}-0.040 \\
(0.032)\end{array}$ & $\begin{array}{l}-0.158 \\
(0.125)\end{array}$ & $\begin{array}{l}0.005 \\
(0.012)\end{array}$ & $\begin{array}{l}0.013 \\
(0.036)\end{array}$ \\
\hline household size & $\begin{array}{l}-0.001 \\
(0.005)\end{array}$ & $\begin{array}{l}-0.000 \\
(0.004)\end{array}$ & $\begin{array}{l}-0.015 \\
(0.005) * *\end{array}$ & $\begin{array}{l}-0.003 \\
(0.012)\end{array}$ & $\begin{array}{l}-0.003 \\
(0.004)\end{array}$ & $\begin{array}{l}0.002 \\
(0.019)\end{array}$ & $\begin{array}{l}-0.002 \\
(0.002)\end{array}$ & $\begin{array}{l}-0.004 \\
(0.004)\end{array}$ \\
\hline Incomplete primary & $\begin{array}{l}0.006 \\
(0.021)\end{array}$ & $\begin{array}{l}0.071 \\
(0.019) * *\end{array}$ & $\begin{array}{l}0.089 \\
(0.024) * *\end{array}$ & $\begin{array}{l}0.151 \\
(0.043) * *\end{array}$ & $\begin{array}{l}0.051 \\
(0.020)^{*}\end{array}$ & $\begin{array}{l}0.109 \\
(0.063)\end{array}$ & $\begin{array}{l}-0.008 \\
(0.008)\end{array}$ & $\begin{array}{l}-0.021 \\
(0.020)\end{array}$ \\
\hline Primary & $\begin{array}{l}0.309 \\
(0.146)^{*}\end{array}$ & $\begin{array}{l}0.096 \\
(0.031) * *\end{array}$ & $\begin{array}{l}0.212 \\
(0.060) * *\end{array}$ & $\begin{array}{l}0.186 \\
(0.073)^{*}\end{array}$ & $\begin{array}{l}0.087 \\
(0.031)^{* *}\end{array}$ & $\begin{array}{l}0.413 \\
(0.162)^{*}\end{array}$ & $\begin{array}{l}-0.030 \\
(0.009)^{* *}\end{array}$ & $\begin{array}{l}-0.065 \\
(0.020) * *\end{array}$ \\
\hline Couple & $\begin{array}{l}0.122 \\
(0.033) * *\end{array}$ & $\begin{array}{l}0.035 \\
(0.040)\end{array}$ & $\begin{array}{l}0.075 \\
(0.043)\end{array}$ & $\begin{array}{l}0.106 \\
(0.076)\end{array}$ & $\begin{array}{l}0.081 \\
(0.040)^{*}\end{array}$ & $\begin{array}{l}0.229 \\
(0.140)\end{array}$ & $\begin{array}{l}0.011 \\
(0.012)\end{array}$ & $\begin{array}{l}-0.033 \\
(0.060)\end{array}$ \\
\hline Divorced/Separated & $\begin{array}{l}0.007 \\
(0.035)\end{array}$ & $\begin{array}{l}0.100 \\
(0.060)\end{array}$ & $\begin{array}{l}0.047 \\
(0.067)\end{array}$ & $\begin{array}{l}0.027 \\
(0.114)\end{array}$ & $\begin{array}{l}0.000 \\
(0.059)\end{array}$ & $\begin{array}{l}0.459 \\
(0.444)\end{array}$ & $\begin{array}{l}-0.010 \\
(0.016)\end{array}$ & $\begin{array}{l}-0.074 \\
(0.062)\end{array}$ \\
\hline
\end{tabular}




\begin{tabular}{|c|c|c|c|c|c|c|c|c|}
\hline Widow & $\begin{array}{l}0.116 \\
(0.041)^{* *}\end{array}$ & $\begin{array}{l}0.033 \\
(0.039)\end{array}$ & $\begin{array}{l}0.068 \\
(0.041)\end{array}$ & $\begin{array}{l}0.053 \\
(0.074)\end{array}$ & $\begin{array}{l}0.106 \\
(0.040)^{* *}\end{array}$ & $\begin{array}{l}0.259 \\
(0.139)\end{array}$ & $\begin{array}{l}0.013 \\
(0.012)\end{array}$ & $\begin{array}{l}-0.028 \\
(0.057)\end{array}$ \\
\hline Constant & $\begin{array}{l}-1.234 \\
(1.620)\end{array}$ & $\begin{array}{l}0.749 \\
(0.941)\end{array}$ & $\begin{array}{l}2.283 \\
(1.338)\end{array}$ & $\begin{array}{l}4.352 \\
(2.277)\end{array}$ & $\begin{array}{l}-0.171 \\
(1.211)\end{array}$ & $\begin{array}{l}-4.095 \\
(3.982)\end{array}$ & $\begin{array}{l}-0.300 \\
(0.309)\end{array}$ & $\begin{array}{l}-1.492 \\
(0.720)^{*}\end{array}$ \\
\hline Observations & 4011 & 4010 & 3983 & 3992 & 4008 & 4004 & 4009 & 4009 \\
\hline R-squared & 0.02 & 0.20 & 0.04 & 0.04 & 0.03 & 0.02 & 0.01 & 0.01 \\
\hline F-test education & 2.79 & 8.34 & 11.14 & 7.48 & 4.98 & 3.76 & 7.58 & 6.92 \\
\hline Prob $>F$ & 0.062 & 0.000 & 0.000 & 0.000 & 0.007 & 0.000 & 0.001 & 0.001 \\
\hline F-test age & 1.665 & 7.905 & 7.522 & 23.314 & 5.861 & 14.688 & 0.883 & 2.987 \\
\hline Prob $>F$ & 0.190 & 0.000 & 0.001 & 0.001 & 0.003 & 0.024 & 0.414 & 0.051 \\
\hline age parabola min/max & 74.540 & 392.061 & 97.452 & 99.180 & 60.147 & 70.483 & 82.505 & 82.457 \\
\hline
\end{tabular}

NOTE: The reference categories are wave 1, Motul, female, do not speak Maya, do not read write a message in Spanish, no schooling, and single. SOURCE: Baseline and first follow-up ENCAHEY, 2008 and 2009.

$* *=$ significant at $5 \%$ level of confidence. $*=$ significant at $10 \%$ level of confidence 
Table 4d: Treatment Regressions

\begin{tabular}{|c|c|c|c|c|c|c|c|}
\hline & $\begin{array}{l}\text { Visited a } \\
\text { dentist } \\
\text { (yes-no [1- } \\
0] \text { ) }\end{array}$ & $\begin{array}{l}\text { Number of } \\
\text { dentist } \\
\text { visits }\end{array}$ & $\begin{array}{l}\text { Outpatient } \\
\text { procedures } \\
\text { (yes-no [1- } \\
0] \text { ) }\end{array}$ & $\begin{array}{l}\text { Consulted a } \\
\text { pharmacist } \\
\text { (yes-no [1- } \\
0] \text { ) }\end{array}$ & $\begin{array}{l}\text { Bought no } \\
\text { medicines } \\
\text { because } \\
\text { they are too } \\
\text { expensive } \\
\text { (yes-no [1- } \\
0] \text { ) }\end{array}$ & $\begin{array}{l}\text { Pay out-of- } \\
\text { pocket (oop) } \\
\text { medical cost } \\
\text { or } \\
\text { medication } \\
\text { (yes-no [1- } \\
0] \text { ) }\end{array}$ & $\begin{array}{l}\text { Oop } \\
\text { expenses } \\
\text { paid by } \\
\text { relatives } \\
\text { (yes-no [1- } \\
0] \text { ) }\end{array}$ \\
\hline Wave & $\begin{array}{l}0.027 \\
(0.017)\end{array}$ & $\begin{array}{l}0.050 \\
(0.049)\end{array}$ & $\begin{array}{l}-0.002 \\
(0.007)\end{array}$ & $\begin{array}{l}-0.084 \\
(0.019)^{* *}\end{array}$ & $\begin{array}{l}-0.147 \\
(0.029)^{* *}\end{array}$ & $\begin{array}{l}-0.045 \\
(0.035)\end{array}$ & $\begin{array}{l}-0.023 \\
(0.029)\end{array}$ \\
\hline Valladolid & $\begin{array}{l}-0.013 \\
(0.012)\end{array}$ & $\begin{array}{l}-0.053 \\
(0.033)\end{array}$ & $\begin{array}{l}-0.001 \\
(0.005)\end{array}$ & $\begin{array}{l}-0.023 \\
(0.011)^{*}\end{array}$ & $\begin{array}{l}-0.040 \\
(0.019)^{*}\end{array}$ & $\begin{array}{l}-0.044 \\
(0.023)\end{array}$ & $\begin{array}{l}-0.102 \\
(0.019)^{* *}\end{array}$ \\
\hline Valladolid wave 2 & $\begin{array}{l}-0.017 \\
(0.016)\end{array}$ & $\begin{array}{l}-0.059 \\
(0.040)\end{array}$ & $\begin{array}{l}-0.004 \\
(0.006)\end{array}$ & $\begin{array}{l}0.004 \\
(0.014)\end{array}$ & $\begin{array}{l}-0.051 \\
(0.023)^{*}\end{array}$ & $\begin{array}{l}0.006 \\
(0.030)\end{array}$ & $\begin{array}{l}-0.110 \\
(0.023)^{* *}\end{array}$ \\
\hline Age & $\begin{array}{l}-0.016 \\
(0.011)\end{array}$ & $\begin{array}{l}-0.032 \\
(0.031)\end{array}$ & $\begin{array}{l}-0.003 \\
(0.005)\end{array}$ & $\begin{array}{l}0.007 \\
(0.010)\end{array}$ & $\begin{array}{l}0.022 \\
(0.017)\end{array}$ & $\begin{array}{l}0.001 \\
(0.027)\end{array}$ & $\begin{array}{l}-0.004 \\
(0.023)\end{array}$ \\
\hline Age squared & $\begin{array}{l}0.000 \\
(0.000)\end{array}$ & $\begin{array}{l}0.000 \\
(0.000)\end{array}$ & $\begin{array}{l}0.000 \\
(0.000)\end{array}$ & $\begin{array}{l}-0.000 \\
(0.000)\end{array}$ & $\begin{array}{l}-0.000 \\
(0.000)\end{array}$ & $\begin{array}{l}-0.000 \\
(0.000)\end{array}$ & $\begin{array}{l}0.000 \\
(0.000)\end{array}$ \\
\hline Gender (male=1) & $\begin{array}{l}-0.019 \\
(0.009) *\end{array}$ & $\begin{array}{l}-0.028 \\
(0.022)\end{array}$ & $\begin{array}{l}0.002 \\
(0.003)\end{array}$ & $\begin{array}{l}-0.000 \\
(0.007)\end{array}$ & $\begin{array}{l}-0.027 \\
(0.013)^{*}\end{array}$ & $\begin{array}{l}-0.059 \\
(0.017)^{* *}\end{array}$ & $\begin{array}{l}-0.068 \\
(0.013)^{* *}\end{array}$ \\
\hline Speaks Maya & $\begin{array}{l}-0.003 \\
(0.015)\end{array}$ & $\begin{array}{l}-0.014 \\
(0.044)\end{array}$ & $\begin{array}{l}-0.006 \\
(0.006)\end{array}$ & $\begin{array}{l}-0.070 \\
(0.016)^{* *}\end{array}$ & $\begin{array}{l}-0.078 \\
(0.024)^{* *}\end{array}$ & $\begin{array}{l}-0.015 \\
(0.027)\end{array}$ & $\begin{array}{l}0.030 \\
(0.022)\end{array}$ \\
\hline Reads/writes Spanish & $\begin{array}{l}0.030 \\
(0.013) *\end{array}$ & $\begin{array}{l}0.077 \\
(0.033) *\end{array}$ & $\begin{array}{l}0.007 \\
(0.005)\end{array}$ & $\begin{array}{l}-0.019 \\
(0.013)\end{array}$ & $\begin{array}{l}-0.048 \\
(0.020)^{*}\end{array}$ & $\begin{array}{l}-0.001 \\
(0.025)\end{array}$ & $\begin{array}{l}-0.051 \\
(0.021)^{*}\end{array}$ \\
\hline Lives alone & $\begin{array}{l}0.024 \\
(0.018)\end{array}$ & $\begin{array}{l}0.063 \\
(0.048)\end{array}$ & $\begin{array}{l}-0.005 \\
(0.005)\end{array}$ & $\begin{array}{l}0.008 \\
(0.013)\end{array}$ & $\begin{array}{l}-0.008 \\
(0.023)\end{array}$ & $\begin{array}{l}-0.070 \\
(0.030) *\end{array}$ & $\begin{array}{l}-0.059 \\
(0.023)^{*}\end{array}$ \\
\hline household size & $\begin{array}{l}-0.003 \\
(0.002)\end{array}$ & $\begin{array}{l}-0.002 \\
(0.008)\end{array}$ & $\begin{array}{l}-0.001 \\
(0.001)\end{array}$ & $\begin{array}{l}-0.002 \\
(0.002)\end{array}$ & $\begin{array}{l}0.004 \\
(0.004)\end{array}$ & $\begin{array}{l}-0.003 \\
(0.004)\end{array}$ & $\begin{array}{l}0.010 \\
(0.004)^{* *}\end{array}$ \\
\hline Incomplete primary & $\begin{array}{l}0.020 \\
(0.009)^{*}\end{array}$ & $\begin{array}{l}0.049 \\
(0.020) *\end{array}$ & $\begin{array}{l}-0.006 \\
(0.004)\end{array}$ & $\begin{array}{l}-0.028 \\
(0.009) * *\end{array}$ & $\begin{array}{l}-0.035 \\
(0.014)^{*}\end{array}$ & $\begin{array}{l}0.026 \\
(0.020)\end{array}$ & $\begin{array}{l}0.020 \\
(0.015)\end{array}$ \\
\hline Primary & $\begin{array}{l}0.060 \\
(0.018) * *\end{array}$ & $\begin{array}{l}0.163 \\
(0.048) * *\end{array}$ & $\begin{array}{l}-0.000 \\
(0.007)\end{array}$ & $\begin{array}{l}-0.050 \\
(0.012) * *\end{array}$ & $\begin{array}{l}-0.030 \\
(0.022)\end{array}$ & $\begin{array}{l}0.060 \\
(0.030)^{*}\end{array}$ & $\begin{array}{l}0.002 \\
(0.022)\end{array}$ \\
\hline Couple & $\begin{array}{l}0.017 \\
(0.019)\end{array}$ & $\begin{array}{l}0.063 \\
(0.036)\end{array}$ & $\begin{array}{l}0.012 \\
(0.003)^{* *}\end{array}$ & $\begin{array}{l}0.008 \\
(0.017)\end{array}$ & $\begin{array}{l}0.004 \\
(0.028)\end{array}$ & $\begin{array}{l}-0.079 \\
(0.039) *\end{array}$ & $\begin{array}{l}-0.108 \\
(0.034)^{* *}\end{array}$ \\
\hline Divorced/Separated & 0.039 & 0.125 & 0.009 & -0.018 & 0.090 & 0.094 & 0.034 \\
\hline
\end{tabular}




\begin{tabular}{llllllll}
\hline & $(0.034)$ & $(0.092)$ & $(0.008)$ & $(0.023)$ & $(0.049)$ & $(0.055)$ & $(0.052)$ \\
Widow & -0.004 & 0.031 & 0.008 & -0.004 & -0.019 & -0.042 & -0.011 \\
& $(0.019)$ & $(0.035)$ & $(0.002)^{* *}$ & $(0.016)$ & $(0.027)$ & $(0.039)$ & $(0.034)$ \\
Constant & 0.753 & 1.495 & 0.136 & -0.057 & -0.408 & 0.514 & 0.435 \\
& $(0.456)$ & $(1.257)$ & $(0.211)$ & $(0.427)$ & $(0.709)$ & $(1.102)$ & $(0.943)$ \\
Observations & 4008 & 4006 & 4008 & 4003 & 4006 & 4007 & 4006 \\
R-squared & 0.02 & 0.01 & 0.00 & 0.02 & 0.03 & 0.01 & 0.07 \\
F-test education & 6.08 & 7.37 & 1.45 & 8.43 & 3.01 & 2.15 & 0.96 \\
Prob>F & 0.002 & 0.119 & 0.236 & 0.000 & 0.049 & 0.932 & 0.001 \\
F-test age & 2.745 & 2.128 & 0.301 & 1.349 & 3.695 & 0.070 & 7.463 \\
Prob>F & 0.065 & 0.001 & 0.740 & 0.260 & 0.025 & 0.117 & 0.382 \\
age parabola min/max & 89.454 & 89.312 & 78.435 & 74.166 & 74.804 & 125.485 & 38.875 \\
\hline
\end{tabular}

NOTE: The reference categories are wave 1, Motul, female, do not speak Maya, do not read write a message in Spanish, no schooling, and single. The model for the number of outpatient procedures could not be estimated due to the few number of observations.

SOURCE: Baseline and first follow-up ENCAHEY, 2008 and 2009.

$* *=$ significant at $5 \%$ level of confidence. ${ }^{*}=$ significant at $10 \%$ level of confidence

Table 4e: Treatment regressions

\begin{tabular}{|c|c|c|c|c|c|c|c|c|}
\hline & $\begin{array}{l}\text { Oop } \\
\text { expenses } \\
\text { paid by } \\
\text { elderly } \\
\text { eligible (yes- } \\
\text { no [1-0]) }\end{array}$ & $\begin{array}{l}\text { Serious } \\
\text { health } \\
\text { problem but } \\
\text { did not go to } \\
\text { the doctor } \\
\text { (yes-no[1-0]) }\end{array}$ & $\begin{array}{l}\text { Did not go to } \\
\text { the doctor } \\
\text { because of } \\
\text { money (yes- } \\
\text { no[1-0]) }\end{array}$ & $\begin{array}{l}\text { Relatives or } \\
\text { friends pay } \\
\text { your } \\
\text { expenses } \\
\text { (not true, } \\
\text { sometimes } \\
\text { true, often } \\
\text { true, al }\end{array}$ & $\begin{array}{l}\text { Feel a } \\
\text { burden on } \\
\text { your family } \\
\text { or friends } \\
\text { (not true, } \\
\text { sometimes } \\
\text { true, often } \\
\text { true, a }\end{array}$ & $\begin{array}{l}\text { Activities you } \\
\text { used to do but } \\
\text { can't do } \\
\text { because of } \\
\text { lack of money } \\
\text { (yes-no[1-0]) }\end{array}$ & $\begin{array}{l}\text { Donation to } \\
\text { the church } \\
\text { but can't do } \\
\text { due to } \\
\text { money (yes- } \\
\text { no[1-0]) }\end{array}$ & $\begin{array}{l}\text { Donation to } \\
\text { local } \\
\text { parties but } \\
\text { can't do due } \\
\text { to money } \\
\text { (yes-no[1- } \\
0] \text { ) }\end{array}$ \\
\hline Wave & $\begin{array}{l}-0.030 \\
(0.028)\end{array}$ & $\begin{array}{l}-0.109 \\
(0.024)^{* *}\end{array}$ & $\begin{array}{l}-0.091 \\
(0.020) * *\end{array}$ & $\begin{array}{l}0.075 \\
(0.077)\end{array}$ & $\begin{array}{l}-0.329 \\
(0.151)^{*}\end{array}$ & $\begin{array}{l}-0.149 \\
(0.036) * *\end{array}$ & $\begin{array}{l}-0.016 \\
(0.008) *\end{array}$ & $\begin{array}{l}-0.006 \\
(0.005)\end{array}$ \\
\hline Valladolid & $\begin{array}{l}0.050 \\
(0.019) * *\end{array}$ & $\begin{array}{l}-0.047 \\
(0.016) * *\end{array}$ & $\begin{array}{l}-0.020 \\
(0.014)\end{array}$ & $\begin{array}{l}-0.107 \\
(0.057)\end{array}$ & $\begin{array}{l}0.103 \\
(0.105)\end{array}$ & $\begin{array}{l}-0.097 \\
(0.025)^{* *}\end{array}$ & $\begin{array}{l}-0.005 \\
(0.006)\end{array}$ & $\begin{array}{l}-0.002 \\
(0.004)\end{array}$ \\
\hline Valladolid wave 2 & $\begin{array}{l}0.086 \\
(0.025) * *\end{array}$ & $\begin{array}{l}-0.024 \\
(0.019)\end{array}$ & $\begin{array}{l}-0.014 \\
(0.016)\end{array}$ & $\begin{array}{l}-0.030 \\
(0.066)\end{array}$ & $\begin{array}{l}0.295 \\
(0.149)^{*}\end{array}$ & $\begin{array}{l}-0.019 \\
(0.031)\end{array}$ & $\begin{array}{l}-0.000 \\
(0.008)\end{array}$ & $\begin{array}{l}-0.002 \\
(0.004)\end{array}$ \\
\hline Age & $\begin{array}{l}0.004 \\
(0.017)\end{array}$ & $\begin{array}{l}-0.000 \\
(0.013)\end{array}$ & $\begin{array}{l}-0.004 \\
(0.012)\end{array}$ & $\begin{array}{l}0.094 \\
(0.067)\end{array}$ & $\begin{array}{l}0.045 \\
(0.118)\end{array}$ & $\begin{array}{l}-0.048 \\
(0.025)\end{array}$ & $\begin{array}{l}-0.002 \\
(0.005)\end{array}$ & $\begin{array}{l}-0.000 \\
(0.002)\end{array}$ \\
\hline
\end{tabular}




\begin{tabular}{|c|c|c|c|c|c|c|c|c|}
\hline Age squared & $\begin{array}{l}-0.000 \\
(0.000)\end{array}$ & $\begin{array}{l}-0.000 \\
(0.000)\end{array}$ & $\begin{array}{l}0.000 \\
(0.000)\end{array}$ & $\begin{array}{l}-0.000 \\
(0.000)\end{array}$ & $\begin{array}{l}-0.000 \\
(0.001)\end{array}$ & $\begin{array}{l}0.000 \\
(0.000)\end{array}$ & $\begin{array}{l}0.000 \\
(0.000)\end{array}$ & $\begin{array}{l}0.000 \\
(0.000)\end{array}$ \\
\hline Gender (male=1) & $\begin{array}{l}0.027 \\
(0.014)^{*}\end{array}$ & $\begin{array}{l}-0.031 \\
(0.011)^{* *}\end{array}$ & $\begin{array}{l}-0.026 \\
(0.008)^{* *}\end{array}$ & $\begin{array}{l}-0.200 \\
(0.040)^{* *}\end{array}$ & $\begin{array}{l}-0.020 \\
(0.078)\end{array}$ & $\begin{array}{l}0.006 \\
(0.016)\end{array}$ & $\begin{array}{l}-0.014 \\
(0.004)^{* *}\end{array}$ & $\begin{array}{l}0.003 \\
(0.002)\end{array}$ \\
\hline Speaks Maya & $\begin{array}{l}-0.048 \\
(0.022)^{*}\end{array}$ & $\begin{array}{l}-0.029 \\
(0.020)\end{array}$ & $\begin{array}{l}-0.035 \\
(0.018)^{*}\end{array}$ & $\begin{array}{l}0.136 \\
(0.064)^{*}\end{array}$ & $\begin{array}{l}-0.208 \\
(0.126)\end{array}$ & $\begin{array}{l}-0.014 \\
(0.028)\end{array}$ & $\begin{array}{l}-0.010 \\
(0.009)\end{array}$ & $\begin{array}{l}-0.004 \\
(0.005)\end{array}$ \\
\hline Reads/writes Spanish & $\begin{array}{l}0.029 \\
(0.019)\end{array}$ & $\begin{array}{l}-0.043 \\
(0.018)^{*}\end{array}$ & $\begin{array}{l}-0.027 \\
(0.015)\end{array}$ & $\begin{array}{l}-0.030 \\
(0.057)\end{array}$ & $\begin{array}{l}-0.047 \\
(0.116)\end{array}$ & $\begin{array}{l}-0.013 \\
(0.027)\end{array}$ & $\begin{array}{l}0.001 \\
(0.008)\end{array}$ & $\begin{array}{l}0.001 \\
(0.004)\end{array}$ \\
\hline Lives alone & $\begin{array}{l}0.007 \\
(0.025)\end{array}$ & $\begin{array}{l}0.008 \\
(0.018)\end{array}$ & $\begin{array}{l}-0.004 \\
(0.013)\end{array}$ & $\begin{array}{l}-0.175 \\
(0.077)^{*}\end{array}$ & $\begin{array}{l}0.245 \\
(0.135)\end{array}$ & $\begin{array}{l}-0.013 \\
(0.028)\end{array}$ & $\begin{array}{l}0.011 \\
(0.008)\end{array}$ & $\begin{array}{l}0.002 \\
(0.004)\end{array}$ \\
\hline household size & $\begin{array}{l}-0.011 \\
(0.004)^{* *}\end{array}$ & $\begin{array}{l}0.000 \\
(0.002)\end{array}$ & $\begin{array}{l}0.000 \\
(0.002)\end{array}$ & $\begin{array}{l}0.059 \\
(0.012)^{* *}\end{array}$ & $\begin{array}{l}0.027 \\
(0.019)\end{array}$ & $\begin{array}{l}0.008 \\
(0.005)\end{array}$ & $\begin{array}{l}-0.001 \\
(0.001)\end{array}$ & $\begin{array}{l}0.000 \\
(0.001)\end{array}$ \\
\hline Incomplete primary & $\begin{array}{l}0.015 \\
(0.016)\end{array}$ & $\begin{array}{l}0.021 \\
(0.012)\end{array}$ & $\begin{array}{l}-0.002 \\
(0.010)\end{array}$ & $\begin{array}{l}-0.086 \\
(0.047)\end{array}$ & $\begin{array}{l}-0.032 \\
(0.093)\end{array}$ & $\begin{array}{l}0.015 \\
(0.019)\end{array}$ & $\begin{array}{l}0.004 \\
(0.005)\end{array}$ & $\begin{array}{l}-0.002 \\
(0.002)\end{array}$ \\
\hline Primary & $\begin{array}{l}0.061 \\
(0.027)^{*}\end{array}$ & $\begin{array}{l}-0.016 \\
(0.017)\end{array}$ & $\begin{array}{l}-0.033 \\
(0.013)^{*}\end{array}$ & $\begin{array}{l}-0.164 \\
(0.075) *\end{array}$ & $\begin{array}{l}-0.321 \\
(0.130)^{*}\end{array}$ & $\begin{array}{l}-0.033 \\
(0.028)\end{array}$ & $\begin{array}{l}-0.007 \\
(0.006)\end{array}$ & $\begin{array}{l}-0.001 \\
(0.004)\end{array}$ \\
\hline Couple & $\begin{array}{l}0.042 \\
(0.030)\end{array}$ & $\begin{array}{l}0.036 \\
(0.020)\end{array}$ & $\begin{array}{l}0.026 \\
(0.017)\end{array}$ & $\begin{array}{l}-0.391 \\
(0.106)^{* *}\end{array}$ & $\begin{array}{l}-0.270 \\
(0.166)\end{array}$ & $\begin{array}{l}0.042 \\
(0.033)\end{array}$ & $\begin{array}{l}0.004 \\
(0.009)\end{array}$ & $\begin{array}{l}0.000 \\
(0.005)\end{array}$ \\
\hline Divorced/Separated & $\begin{array}{l}0.061 \\
(0.050)\end{array}$ & $\begin{array}{l}0.026 \\
(0.035)\end{array}$ & $\begin{array}{l}0.004 \\
(0.025)\end{array}$ & $\begin{array}{l}0.025 \\
(0.159)\end{array}$ & $\begin{array}{l}-0.459 \\
(0.219)^{*}\end{array}$ & $\begin{array}{l}0.067 \\
(0.055)\end{array}$ & $\begin{array}{l}-0.017 \\
(0.008)^{*}\end{array}$ & $\begin{array}{l}-0.005 \\
(0.005)\end{array}$ \\
\hline Widow & $\begin{array}{l}-0.023 \\
(0.029)\end{array}$ & $\begin{array}{l}0.016 \\
(0.020)\end{array}$ & $\begin{array}{l}0.011 \\
(0.016)\end{array}$ & $\begin{array}{l}0.043 \\
(0.104)\end{array}$ & $\begin{array}{l}-0.255 \\
(0.161)\end{array}$ & $\begin{array}{l}0.023 \\
(0.032)\end{array}$ & $\begin{array}{l}0.003 \\
(0.009)\end{array}$ & $\begin{array}{l}-0.001 \\
(0.005)\end{array}$ \\
\hline Constant & $\begin{array}{l}0.094 \\
(0.721)\end{array}$ & $\begin{array}{l}0.322 \\
(0.553)\end{array}$ & $\begin{array}{l}0.384 \\
(0.477)\end{array}$ & $\begin{array}{l}-2.176 \\
(2.722)\end{array}$ & $\begin{array}{l}0.431 \\
(4.791)\end{array}$ & $\begin{array}{l}2.439 \\
(1.027)^{*}\end{array}$ & $\begin{array}{l}0.125 \\
(0.204)\end{array}$ & $\begin{array}{l}0.022 \\
(0.103)\end{array}$ \\
\hline Observations & 4006 & 3999 & 4011 & 3996 & 910 & 3254 & 4011 & 4011 \\
\hline R-squared & 0.03 & 0.03 & 0.02 & 0.09 & 0.03 & 0.04 & 0.01 & 0.00 \\
\hline F-test education & 2.54 & 3.45 & 4.33 & 2.88 & 3.63 & 1.78 & 2.28 & 0.42 \\
\hline Prob $>F$ & 0.079 & 0.022 & 0.013 & 0.000 & 0.875 & 0.079 & 0.007 & 0.658 \\
\hline F-test age & 6.118 & 3.848 & 1.746 & 17.315 & 0.133 & 2.541 & 5.013 & 0.006 \\
\hline Prob $>F$ & 0.002 & 0.032 & 0.175 & 0.057 & 0.027 & 0.169 & 0.103 & 0.994 \\
\hline age parabola min/max & 43.783 & -0.838 & 114.568 & 104.679 & 78.624 & 84.864 & 157.096 & 83.646 \\
\hline
\end{tabular}

NOTE: The reference categories are wave 1, Motul, female, do not speak Maya, do not read write a message in Spanish, no schooling, and single.

SOURCE: Baseline and first follow-up ENCAHEY, 2008 and 2009.

$* *=$ significant at $5 \%$ level of confidence. $*=$ significant at $10 \%$ level of confidence 
Table 4f: Treatment regressions

\begin{tabular}{|c|c|c|c|c|c|c|c|c|}
\hline & $\begin{array}{l}\text { Donation to } \\
\text { family or } \\
\text { friends } \\
\text { parties but } \\
\text { can't do due } \\
\text { to money } \\
\text { (yes-no[1- } \\
0] \text { ) }\end{array}$ & $\begin{array}{l}\text { Community } \\
\text { activities but } \\
\text { can't do due to } \\
\text { money (yes- } \\
\text { no[1-0]) }\end{array}$ & $\begin{array}{l}\text { Help out } \\
\text { family } \\
\text { members but } \\
\text { can't do due } \\
\text { to money } \\
\text { (yes-no[1-0]) }\end{array}$ & $\begin{array}{l}\text { Help out } \\
\text { non-relatives } \\
\text { but can't do } \\
\text { due to } \\
\text { money (yes- } \\
\text { no[1-0]) }\end{array}$ & $\begin{array}{l}\text { Travel to } \\
\text { visit family } \\
\text { or friends but } \\
\text { can't do due } \\
\text { to money } \\
\text { (yes-no[1-0]) }\end{array}$ & $\begin{array}{l}\text { Sometimes } \\
\text { do not have } \\
\text { enough to eat } \\
\text { (yes-no[1-0]) }\end{array}$ & $\begin{array}{l}\text { Often we do } \\
\text { not have } \\
\text { enough to eat } \\
\text { (yes-no[1-0]) }\end{array}$ & $\begin{array}{l}\text { Sometimes } \\
\text { or often we } \\
\text { don't have } \\
\text { enough to eat } \\
\text { because of } \\
\text { money (yes- } \\
\text { no[1-0]) }\end{array}$ \\
\hline Wave & $\begin{array}{l}-0.005 \\
(0.008)\end{array}$ & $\begin{array}{l}-0.002 \\
(0.007)\end{array}$ & $\begin{array}{l}-0.033 \\
(0.023)\end{array}$ & $\begin{array}{l}-0.036 \\
(0.012)^{* *}\end{array}$ & $\begin{array}{l}-0.024 \\
(0.014)\end{array}$ & $\begin{array}{l}-0.162 \\
(0.035)^{* *}\end{array}$ & $\begin{array}{l}-0.051 \\
(0.019) * *\end{array}$ & $\begin{array}{l}0.039 \\
(0.033)\end{array}$ \\
\hline Valladolid & $\begin{array}{l}0.001 \\
(0.007)\end{array}$ & $\begin{array}{l}-0.014 \\
(0.005)^{* *}\end{array}$ & $\begin{array}{l}-0.018 \\
(0.016)\end{array}$ & $\begin{array}{l}-0.013 \\
(0.008)\end{array}$ & $\begin{array}{l}-0.029 \\
(0.010) * *\end{array}$ & $\begin{array}{l}0.062 \\
(0.025)^{*}\end{array}$ & $\begin{array}{l}0.019 \\
(0.013)\end{array}$ & $\begin{array}{l}0.103 \\
(0.025) * *\end{array}$ \\
\hline Valladolid wave 2 & $\begin{array}{l}0.003 \\
(0.009)\end{array}$ & $\begin{array}{l}-0.009 \\
(0.007)\end{array}$ & $\begin{array}{l}0.025 \\
(0.021)\end{array}$ & $\begin{array}{l}-0.004 \\
(0.008)\end{array}$ & $\begin{array}{l}-0.020 \\
(0.012)\end{array}$ & $\begin{array}{l}-0.010 \\
(0.031)\end{array}$ & $\begin{array}{l}0.026 \\
(0.016)\end{array}$ & $\begin{array}{l}0.035 \\
(0.030)\end{array}$ \\
\hline Age & $\begin{array}{l}-0.005 \\
(0.005)\end{array}$ & $\begin{array}{l}-0.003 \\
(0.004)\end{array}$ & $\begin{array}{l}-0.016 \\
(0.015)\end{array}$ & $\begin{array}{l}0.002 \\
(0.006)\end{array}$ & $\begin{array}{l}0.005 \\
(0.007)\end{array}$ & $\begin{array}{l}0.005 \\
(0.027)\end{array}$ & $\begin{array}{l}0.001 \\
(0.010)\end{array}$ & $\begin{array}{l}-0.003 \\
(0.027)\end{array}$ \\
\hline Age squared & $\begin{array}{l}0.000 \\
(0.000)\end{array}$ & $\begin{array}{l}0.000 \\
(0.000)\end{array}$ & $\begin{array}{l}0.000 \\
(0.000)\end{array}$ & $\begin{array}{l}-0.000 \\
(0.000)\end{array}$ & $\begin{array}{l}-0.000 \\
(0.000)\end{array}$ & $\begin{array}{l}-0.000 \\
(0.000)\end{array}$ & $\begin{array}{l}-0.000 \\
(0.000)\end{array}$ & $\begin{array}{l}-0.000 \\
(0.000)\end{array}$ \\
\hline Gender (male=1) & $\begin{array}{l}-0.005 \\
(0.005)\end{array}$ & $\begin{array}{l}0.004 \\
(0.003)\end{array}$ & $\begin{array}{l}0.015 \\
(0.011)\end{array}$ & $\begin{array}{l}0.003 \\
(0.004)\end{array}$ & $\begin{array}{l}0.002 \\
(0.006)\end{array}$ & $\begin{array}{l}0.008 \\
(0.013)\end{array}$ & $\begin{array}{l}-0.000 \\
(0.006)\end{array}$ & $\begin{array}{l}-0.006 \\
(0.013)\end{array}$ \\
\hline Speaks Maya & $\begin{array}{l}0.007 \\
(0.008)\end{array}$ & $\begin{array}{l}-0.010 \\
(0.007)\end{array}$ & $\begin{array}{l}-0.010 \\
(0.018)\end{array}$ & $\begin{array}{l}-0.020 \\
(0.011)\end{array}$ & $\begin{array}{l}-0.016 \\
(0.013)\end{array}$ & $\begin{array}{l}-0.067 \\
(0.027)^{*}\end{array}$ & $\begin{array}{l}-0.011 \\
(0.015)\end{array}$ & $\begin{array}{l}0.125 \\
(0.024) * *\end{array}$ \\
\hline Reads/writes Spanish & $\begin{array}{l}0.012 \\
(0.007)\end{array}$ & $\begin{array}{l}0.008 \\
(0.005)\end{array}$ & $\begin{array}{l}-0.014 \\
(0.017)\end{array}$ & $\begin{array}{l}-0.007 \\
(0.009)\end{array}$ & $\begin{array}{l}0.014 \\
(0.010)\end{array}$ & $\begin{array}{l}-0.066 \\
(0.024)^{* *}\end{array}$ & $\begin{array}{l}-0.012 \\
(0.012)\end{array}$ & $\begin{array}{l}-0.010 \\
(0.024)\end{array}$ \\
\hline Lives alone & $\begin{array}{l}0.015 \\
(0.008)\end{array}$ & $\begin{array}{l}0.004 \\
(0.006)\end{array}$ & $\begin{array}{l}-0.012 \\
(0.019)\end{array}$ & $\begin{array}{l}0.004 \\
(0.008)\end{array}$ & $\begin{array}{l}0.004 \\
(0.010)\end{array}$ & $\begin{array}{l}0.007 \\
(0.027)\end{array}$ & $\begin{array}{l}0.024 \\
(0.014)\end{array}$ & $\begin{array}{l}0.050 \\
(0.028)\end{array}$ \\
\hline household size & $\begin{array}{l}0.003 \\
(0.001)^{*}\end{array}$ & $\begin{array}{l}0.000 \\
(0.001)\end{array}$ & $\begin{array}{l}0.003 \\
(0.003)\end{array}$ & $\begin{array}{l}-0.000 \\
(0.001)\end{array}$ & $\begin{array}{l}0.003 \\
(0.002)\end{array}$ & $\begin{array}{l}0.002 \\
(0.005)\end{array}$ & $\begin{array}{l}0.001 \\
(0.002)\end{array}$ & $\begin{array}{l}0.004 \\
(0.005)\end{array}$ \\
\hline Incomplete primary & $\begin{array}{l}-0.009 \\
(0.005)\end{array}$ & $\begin{array}{l}0.000 \\
(0.004)\end{array}$ & $\begin{array}{l}0.005 \\
(0.013)\end{array}$ & $\begin{array}{l}0.000 \\
(0.005)\end{array}$ & $\begin{array}{l}-0.001 \\
(0.007)\end{array}$ & $\begin{array}{l}-0.047 \\
(0.018)^{* *}\end{array}$ & $\begin{array}{l}-0.021 \\
(0.010)^{*}\end{array}$ & $\begin{array}{l}-0.054 \\
(0.018) * *\end{array}$ \\
\hline Primary & $\begin{array}{l}-0.002 \\
(0.008)\end{array}$ & $\begin{array}{l}-0.003 \\
(0.006)\end{array}$ & $\begin{array}{l}-0.050 \\
(0.018)^{* *}\end{array}$ & $\begin{array}{l}-0.004 \\
(0.008)\end{array}$ & $\begin{array}{l}0.003 \\
(0.011)\end{array}$ & $\begin{array}{l}-0.162 \\
(0.025)^{* *}\end{array}$ & $\begin{array}{l}-0.029 \\
(0.012)^{*}\end{array}$ & $\begin{array}{l}-0.147 \\
(0.026)^{* *}\end{array}$ \\
\hline Couple & 0.012 & 0.004 & -0.002 & -0.019 & -0.007 & -0.068 & 0.011 & -0.038 \\
\hline
\end{tabular}




\begin{tabular}{|c|c|c|c|c|c|c|c|c|}
\hline & $(0.008)$ & $(0.007)$ & $(0.025)$ & $(0.013)$ & $(0.014)$ & $(0.042)$ & $(0.017)$ & $(0.037)$ \\
\hline \multirow[t]{2}{*}{ Divorced/Separated } & 0.020 & 0.006 & 0.015 & 0.003 & 0.014 & -0.064 & 0.024 & -0.010 \\
\hline & $(0.017)$ & $(0.013)$ & $(0.045)$ & $(0.022)$ & $(0.024)$ & $(0.054)$ & $(0.029)$ & $(0.053)$ \\
\hline \multirow[t]{2}{*}{ Widow } & 0.008 & -0.001 & -0.015 & -0.020 & -0.014 & -0.105 & -0.006 & -0.083 \\
\hline & (0.008) & $(0.007)$ & $(0.024)$ & $(0.013)$ & $(0.014)$ & $(0.040)^{* *}$ & $(0.016)$ & $(0.036)^{*}$ \\
\hline \multirow[t]{2}{*}{ Constant } & 0.249 & 0.164 & 0.919 & 0.039 & -0.066 & 0.335 & 0.023 & 0.379 \\
\hline & $(0.222)$ & $(0.171)$ & $(0.634)$ & $(0.233)$ & $(0.312)$ & (1.090) & $(0.428)$ & (1.094) \\
\hline Observations & 4011 & 4011 & 4011 & 4011 & 4011 & 4011 & 4011 & 4011 \\
\hline R-squared & 0.01 & 0.01 & 0.01 & 0.01 & 0.02 & 0.04 & 0.01 & 0.03 \\
\hline F-test education & 1.93 & 0.19 & 5.93 & 0.19 & 0.08 & 21.03 & 3.22 & 16.18 \\
\hline Prob $>F$ & 0.146 & 0.827 & 0.003 & 0.086 & 0.088 & 0.000 & 0.997 & 0.121 \\
\hline F-test age & 5.295 & 0.641 & 5.908 & 2.452 & 2.435 & 1.581 & 0.003 & 2.114 \\
\hline Prob $>F$ & 0.005 & 0.527 & 0.003 & 0.830 & 0.920 & 0.206 & 0.040 & 0.000 \\
\hline age parabola min/max & 99.242 & 89.760 & 99.595 & 61.774 & 72.474 & 53.333 & 82.393 & -1028.559 \\
\hline
\end{tabular}

NOTE: The reference categories are wave 1, Motul, female, do not speak Maya, do not read write a message in Spanish, no schooling, and single.

SOURCE: Baseline and first follow-up ENCAHEY, 2008 and 2009.

** = significant at $5 \%$ level of confidence. $*$ = significant at $10 \%$ level of confidence

Table 4g: Treatment regressions

\begin{tabular}{|c|c|c|c|c|c|c|c|c|}
\hline & $\begin{array}{l}\text { Often } \\
\text { worried to } \\
\text { run out of } \\
\text { food last } \\
\text { three months } \\
\text { (never- } \\
\text { always [1-4]) }\end{array}$ & $\begin{array}{l}\text { Run out of } \\
\text { food and } \\
\text { money not } \\
\text { enough } \\
\text { (never- } \\
\text { always [1-4]) }\end{array}$ & $\begin{array}{l}\text { Skip or cut } \\
\text { meals } \\
\text { (never- } \\
\text { always [1-4]) }\end{array}$ & $\begin{array}{l}\text { Often eat } \\
\text { less than you } \\
\text { felt you } \\
\text { should } \\
\text { (never- } \\
\text { always [1-4]) }\end{array}$ & $\begin{array}{l}\text { Often hungry } \\
\text { (never- } \\
\text { always [1-4]) }\end{array}$ & $\begin{array}{l}\text { Not eat all } \\
\text { day (never- } \\
\text { always [1-4]) }\end{array}$ & $\begin{array}{l}\text { Food from } \\
\text { charity } \\
\text { (never- } \\
\text { always [1-4]) }\end{array}$ & $\begin{array}{l}\text { Spend on food } \\
\text { at home last } \\
\text { week }\end{array}$ \\
\hline Wave & $\begin{array}{l}-0.411 \\
(0.069)^{* *}\end{array}$ & $\begin{array}{l}-0.252 \\
(0.059)^{* *}\end{array}$ & $\begin{array}{l}-0.299 \\
(0.058)^{* *}\end{array}$ & $\begin{array}{l}-0.238 \\
(0.054)^{* *}\end{array}$ & $\begin{array}{l}-0.263 \\
(0.050)^{* *}\end{array}$ & $\begin{array}{l}-0.163 \\
(0.039) * *\end{array}$ & $\begin{array}{l}-0.053 \\
(0.024)^{*}\end{array}$ & $\begin{array}{l}35.325 \\
(22.248)\end{array}$ \\
\hline Valladolid & $\begin{array}{l}-0.007 \\
(0.049)\end{array}$ & $\begin{array}{l}-0.129 \\
(0.041)^{* *}\end{array}$ & $\begin{array}{l}-0.065 \\
(0.041)\end{array}$ & $\begin{array}{l}-0.064 \\
(0.038)\end{array}$ & $\begin{array}{l}-0.102 \\
(0.033) * *\end{array}$ & $\begin{array}{l}-0.080 \\
(0.024)^{* *}\end{array}$ & $\begin{array}{l}-0.034 \\
(0.015)^{*}\end{array}$ & $\begin{array}{l}-21.703 \\
(17.810)\end{array}$ \\
\hline Valladolid wave 2 & $\begin{array}{l}0.116 \\
(0.061)\end{array}$ & $\begin{array}{l}-0.139 \\
(0.052)^{* *}\end{array}$ & $\begin{array}{l}-0.071 \\
(0.050)\end{array}$ & $\begin{array}{l}-0.099 \\
(0.047)^{*}\end{array}$ & $\begin{array}{l}-0.092 \\
(0.038) *\end{array}$ & $\begin{array}{l}-0.100 \\
(0.028) * *\end{array}$ & $\begin{array}{l}-0.050 \\
(0.019) * *\end{array}$ & $\begin{array}{l}18.869 \\
(22.229)\end{array}$ \\
\hline Age & $\begin{array}{l}-0.093 \\
(0.061)\end{array}$ & $\begin{array}{l}-0.109 \\
(0.052)^{*}\end{array}$ & $\begin{array}{l}-0.062 \\
(0.052)\end{array}$ & $\begin{array}{l}-0.121 \\
(0.043)^{* *}\end{array}$ & $\begin{array}{l}-0.091 \\
(0.041)^{*}\end{array}$ & $\begin{array}{l}-0.044 \\
(0.033)\end{array}$ & $\begin{array}{l}-0.003 \\
(0.016)\end{array}$ & $\begin{array}{l}15.747 \\
(24.308)\end{array}$ \\
\hline
\end{tabular}




\begin{tabular}{|c|c|c|c|c|c|c|c|c|}
\hline Age squared & $\begin{array}{l}0.001 \\
(0.000)\end{array}$ & $\begin{array}{l}0.001 \\
(0.000)^{*}\end{array}$ & $\begin{array}{l}0.000 \\
(0.000)\end{array}$ & $\begin{array}{l}0.001 \\
(0.000) * *\end{array}$ & $\begin{array}{l}0.001 \\
(0.000)^{*}\end{array}$ & $\begin{array}{l}0.000 \\
(0.000)\end{array}$ & $\begin{array}{l}0.000 \\
(0.000)\end{array}$ & $\begin{array}{l}-0.098 \\
(0.150)\end{array}$ \\
\hline Gender (male $=1$ ) & $\begin{array}{l}0.049 \\
(0.027)\end{array}$ & $\begin{array}{l}0.057 \\
(0.023)^{*}\end{array}$ & $\begin{array}{l}0.039 \\
(0.021)\end{array}$ & $\begin{array}{l}0.056 \\
(0.021)^{* *}\end{array}$ & $\begin{array}{l}0.061 \\
(0.018) * *\end{array}$ & $\begin{array}{l}0.027 \\
(0.013)^{*}\end{array}$ & $\begin{array}{l}0.003 \\
(0.009)\end{array}$ & $\begin{array}{l}-17.353 \\
(9.317)\end{array}$ \\
\hline Speaks Maya & $\begin{array}{l}-0.108 \\
(0.055)^{*}\end{array}$ & $\begin{array}{l}-0.108 \\
(0.048) *\end{array}$ & $\begin{array}{l}-0.059 \\
(0.047)\end{array}$ & $\begin{array}{l}-0.058 \\
(0.045)\end{array}$ & $\begin{array}{l}-0.082 \\
(0.040)^{*}\end{array}$ & $\begin{array}{l}-0.061 \\
(0.031) *\end{array}$ & $\begin{array}{l}-0.051 \\
(0.022)^{*}\end{array}$ & $\begin{array}{l}12.071 \\
(20.207)\end{array}$ \\
\hline Reads/writes Spanish & $\begin{array}{l}-0.144 \\
(0.051)^{* *}\end{array}$ & $\begin{array}{l}-0.115 \\
(0.041)^{* *}\end{array}$ & $\begin{array}{l}-0.158 \\
(0.042)^{* *}\end{array}$ & $\begin{array}{l}-0.087 \\
(0.038)^{*}\end{array}$ & $\begin{array}{l}-0.100 \\
(0.037)^{* *}\end{array}$ & $\begin{array}{l}-0.089 \\
(0.026) * *\end{array}$ & $\begin{array}{l}-0.030 \\
(0.017)\end{array}$ & $\begin{array}{l}24.653 \\
(17.426)\end{array}$ \\
\hline Lives alone & $\begin{array}{l}-0.035 \\
(0.055)\end{array}$ & $\begin{array}{l}-0.042 \\
(0.047)\end{array}$ & $\begin{array}{l}-0.046 \\
(0.045)\end{array}$ & $\begin{array}{l}-0.044 \\
(0.042)\end{array}$ & $\begin{array}{l}0.052 \\
(0.037)\end{array}$ & $\begin{array}{l}0.036 \\
(0.027)\end{array}$ & $\begin{array}{l}0.041 \\
(0.019) *\end{array}$ & $\begin{array}{l}-125.042 \\
(20.130)^{* *}\end{array}$ \\
\hline household size & $\begin{array}{l}0.009 \\
(0.009)\end{array}$ & $\begin{array}{l}-0.004 \\
(0.008)\end{array}$ & $\begin{array}{l}0.007 \\
(0.007)\end{array}$ & $\begin{array}{l}0.003 \\
(0.007)\end{array}$ & $\begin{array}{l}0.001 \\
(0.006)\end{array}$ & $\begin{array}{l}-0.003 \\
(0.004)\end{array}$ & $\begin{array}{l}-0.002 \\
(0.002)\end{array}$ & $\begin{array}{l}27.904 \\
(3.816)^{* *}\end{array}$ \\
\hline Incomplete primary & $\begin{array}{l}-0.094 \\
(0.040)^{*}\end{array}$ & $\begin{array}{l}-0.140 \\
(0.033)^{* *}\end{array}$ & $\begin{array}{l}-0.055 \\
(0.033)\end{array}$ & $\begin{array}{l}-0.090 \\
(0.030)^{* *}\end{array}$ & $\begin{array}{l}-0.074 \\
(0.026)^{* *}\end{array}$ & $\begin{array}{l}-0.049 \\
(0.016)^{* *}\end{array}$ & $\begin{array}{l}-0.012 \\
(0.014)\end{array}$ & $\begin{array}{l}35.731 \\
(14.161)^{*}\end{array}$ \\
\hline Primary & $\begin{array}{l}-0.351 \\
(0.055)^{* *}\end{array}$ & $\begin{array}{l}-0.363 \\
(0.047)^{* *}\end{array}$ & $\begin{array}{l}-0.269 \\
(0.042)^{* *}\end{array}$ & $\begin{array}{l}-0.302 \\
(0.037)^{* *}\end{array}$ & $\begin{array}{l}-0.204 \\
(0.032)^{* *}\end{array}$ & $\begin{array}{l}-0.104 \\
(0.021)^{* *}\end{array}$ & $\begin{array}{l}-0.037 \\
(0.015)^{*}\end{array}$ & $\begin{array}{l}179.514 \\
(33.636)^{* *}\end{array}$ \\
\hline Couple & $\begin{array}{l}0.126 \\
(0.079)\end{array}$ & $\begin{array}{l}0.077 \\
(0.054)\end{array}$ & $\begin{array}{l}0.009 \\
(0.062)\end{array}$ & $\begin{array}{l}0.031 \\
(0.056)\end{array}$ & $\begin{array}{l}-0.013 \\
(0.050)\end{array}$ & $\begin{array}{l}-0.026 \\
(0.036)\end{array}$ & $\begin{array}{l}0.024 \\
(0.020)\end{array}$ & $\begin{array}{l}41.721 \\
(26.994)\end{array}$ \\
\hline Divorced/Separated & $\begin{array}{l}0.136 \\
(0.117)\end{array}$ & $\begin{array}{l}0.174 \\
(0.101)\end{array}$ & $\begin{array}{l}0.088 \\
(0.104)\end{array}$ & $\begin{array}{l}0.084 \\
(0.094)\end{array}$ & $\begin{array}{l}0.082 \\
(0.075)\end{array}$ & $\begin{array}{l}0.136 \\
(0.071)\end{array}$ & $\begin{array}{l}-0.008 \\
(0.026)\end{array}$ & $\begin{array}{l}64.195 \\
(36.637)\end{array}$ \\
\hline Widow & $\begin{array}{l}0.086 \\
(0.077)\end{array}$ & $\begin{array}{l}0.021 \\
(0.051)\end{array}$ & $\begin{array}{l}-0.055 \\
(0.059)\end{array}$ & $\begin{array}{l}-0.031 \\
(0.052)\end{array}$ & $\begin{array}{l}-0.033 \\
(0.045)\end{array}$ & $\begin{array}{l}-0.045 \\
(0.034)\end{array}$ & $\begin{array}{l}0.015 \\
(0.019)\end{array}$ & $\begin{array}{l}72.397 \\
(25.624)^{* *}\end{array}$ \\
\hline Constant & $\begin{array}{l}5.633 \\
(2.479) *\end{array}$ & $\begin{array}{l}6.521 \\
(2.085)^{* *}\end{array}$ & $\begin{array}{l}4.313 \\
(2.089) *\end{array}$ & $\begin{array}{l}6.641 \\
(1.758) * *\end{array}$ & $\begin{array}{l}5.365 \\
(1.655)^{* *}\end{array}$ & $\begin{array}{l}3.293 \\
(1.305) *\end{array}$ & $\begin{array}{l}1.208 \\
(0.650)\end{array}$ & $\begin{array}{l}-317.186 \\
(976.605)\end{array}$ \\
\hline Observations & 3624 & 3624 & 3619 & 3623 & 3624 & 3625 & 3630 & 3041 \\
\hline R-squared & 0.04 & 0.06 & 0.05 & 0.05 & 0.06 & 0.06 & 0.02 & 0.11 \\
\hline F-test education & 21.44 & 30.36 & 23.18 & 35.06 & 21.50 & 11.70 & 3.60 & 14.30 \\
\hline Prob $>F$ & 0.000 & 0.000 & 0.000 & 0.000 & 0.056 & 0.000 & 0.027 & 0.000 \\
\hline F-test age & 2.051 & 2.384 & 0.964 & 4.118 & 2.884 & 1.680 & 0.986 & 0.225 \\
\hline Prob $>F$ & 0.129 & 0.093 & 0.382 & 0.016 & 0.000 & 0.187 & 0.373 & 0.798 \\
\hline age parabola $\min / \max$ & 77.555 & 82.226 & 78.688 & 80.565 & 82.350 & 83.422 & 51.237 & 80.243 \\
\hline
\end{tabular}

NOTE: The reference categories are wave 1, Motul, female, do not speak Maya, do not read write a message in Spanish, no schooling, and single.

SOURCE: Baseline and first follow-up ENCAHEY, 2008 and 2009.

$* *=$ significant at $5 \%$ level of confidence. ${ }^{*}=$ significant at $10 \%$ level of confidence 
Table 4h: Treatment regressions

\begin{tabular}{|c|c|c|c|c|c|c|c|c|}
\hline & $\begin{array}{l}\text { Spend on } \\
\text { food away } \\
\text { from home }\end{array}$ & Total food & $\begin{array}{l}\text { Received } \\
\text { free food } \\
\text { (yes-no [1- } \\
0] \text { ) }\end{array}$ & $\begin{array}{l}\text { Eat diary } \\
\text { products: } \\
\text { milk, cheese, } \\
\text { yogurt (at } \\
\text { least once a } \\
\text { day, at least } \\
\text { once a w }\end{array}$ & $\begin{array}{l}\text { Eat eggs, } \\
\text { beans or } \\
\text { lentil (at } \\
\text { least once a } \\
\text { day, at least } \\
\text { once a week, } \\
\text { several ti }\end{array}$ & $\begin{array}{l}\text { Eat meat, } \\
\text { poultry or } \\
\text { fish (at least } \\
\text { once a day, } \\
\text { at least once } \\
\text { a week, } \\
\text { several ti }\end{array}$ & $\begin{array}{l}\text { Eat fruit or } \\
\text { vegetables (at } \\
\text { least once a } \\
\text { day, at least } \\
\text { once a week, } \\
\text { several time }\end{array}$ & $\begin{array}{l}\text { Eat tortillas, } \\
\text { bread, } \\
\text { crackers or } \\
\text { other cereals } \\
\text { (at least once a } \\
\text { day, at least o }\end{array}$ \\
\hline Wave & $\begin{array}{l}6.258 \\
(9.444)\end{array}$ & $\begin{array}{l}39.465 \\
(27.691)\end{array}$ & $\begin{array}{l}0.088 \\
(0.024)^{* *}\end{array}$ & $\begin{array}{l}-0.163 \\
(0.089)\end{array}$ & $\begin{array}{l}0.172 \\
(0.070)^{*}\end{array}$ & $\begin{array}{l}0.076 \\
(0.071)\end{array}$ & $\begin{array}{l}-0.069 \\
(0.075)\end{array}$ & $\begin{array}{l}-0.113 \\
(0.033)^{* *}\end{array}$ \\
\hline Valladolid & $\begin{array}{l}-11.161 \\
(9.170)\end{array}$ & $\begin{array}{l}-37.044 \\
(24.539)\end{array}$ & $\begin{array}{l}-0.046 \\
(0.016)^{* *}\end{array}$ & $\begin{array}{l}0.191 \\
(0.067)^{* *}\end{array}$ & $\begin{array}{l}0.136 \\
(0.049)^{* *}\end{array}$ & $\begin{array}{l}0.017 \\
(0.051)\end{array}$ & $\begin{array}{l}0.004 \\
(0.058)\end{array}$ & $\begin{array}{l}-0.031 \\
(0.021)\end{array}$ \\
\hline Valladolid wave 2 & $\begin{array}{l}-11.726 \\
(17.510)\end{array}$ & $\begin{array}{l}11.128 \\
(37.975)\end{array}$ & $\begin{array}{l}-0.051 \\
(0.023) *\end{array}$ & $\begin{array}{l}-0.010 \\
(0.075)\end{array}$ & $\begin{array}{l}-0.012 \\
(0.062)\end{array}$ & $\begin{array}{l}-0.022 \\
(0.064)\end{array}$ & $\begin{array}{l}-0.007 \\
(0.069)\end{array}$ & $\begin{array}{l}0.002 \\
(0.027)\end{array}$ \\
\hline Age & $\begin{array}{l}-0.015 \\
(12.480)\end{array}$ & $\begin{array}{l}17.364 \\
(34.470)\end{array}$ & $\begin{array}{l}0.035 \\
(0.019)\end{array}$ & $\begin{array}{l}-0.023 \\
(0.087)\end{array}$ & $\begin{array}{l}0.009 \\
(0.048)\end{array}$ & $\begin{array}{l}-0.004 \\
(0.053)\end{array}$ & $\begin{array}{l}-0.060 \\
(0.067)\end{array}$ & $\begin{array}{l}0.014 \\
(0.016)\end{array}$ \\
\hline Age squared & $\begin{array}{l}-0.003 \\
(0.073)\end{array}$ & $\begin{array}{l}-0.111 \\
(0.209)\end{array}$ & $\begin{array}{l}-0.000 \\
(0.000)\end{array}$ & $\begin{array}{l}0.000 \\
(0.001)\end{array}$ & $\begin{array}{l}-0.000 \\
(0.000)\end{array}$ & $\begin{array}{l}0.000 \\
(0.000)\end{array}$ & $\begin{array}{l}0.000 \\
(0.000)\end{array}$ & $\begin{array}{l}-0.000 \\
(0.000)\end{array}$ \\
\hline Gender (male=1) & $\begin{array}{l}-4.557 \\
(3.925)\end{array}$ & $\begin{array}{l}-21.972 \\
(11.153)^{*}\end{array}$ & $\begin{array}{l}0.007 \\
(0.011)\end{array}$ & $\begin{array}{l}0.350 \\
(0.050)^{* *}\end{array}$ & $\begin{array}{l}-0.137 \\
(0.033)^{* *}\end{array}$ & $\begin{array}{l}0.086 \\
(0.034)^{*}\end{array}$ & $\begin{array}{l}0.166 \\
(0.038)^{* *}\end{array}$ & $\begin{array}{l}-0.007 \\
(0.015)\end{array}$ \\
\hline Speaks Maya & $\begin{array}{l}15.202 \\
(9.862)\end{array}$ & $\begin{array}{l}25.127 \\
(28.006)\end{array}$ & $\begin{array}{l}0.032 \\
(0.018)\end{array}$ & $\begin{array}{l}0.237 \\
(0.072)^{* *}\end{array}$ & $\begin{array}{l}0.080 \\
(0.054)\end{array}$ & $\begin{array}{l}0.209 \\
(0.055)^{* *}\end{array}$ & $\begin{array}{l}0.120 \\
(0.061)\end{array}$ & $\begin{array}{l}-0.055 \\
(0.026)^{*}\end{array}$ \\
\hline Reads/writes Spanish & $\begin{array}{l}-13.759 \\
(10.768)\end{array}$ & $\begin{array}{l}5.694 \\
(26.219)\end{array}$ & $\begin{array}{l}0.022 \\
(0.017)\end{array}$ & $\begin{array}{l}-0.383 \\
(0.067)^{* *}\end{array}$ & $\begin{array}{l}0.049 \\
(0.053)\end{array}$ & $\begin{array}{l}-0.171 \\
(0.053)^{* *}\end{array}$ & $\begin{array}{l}-0.195 \\
(0.060)^{* *}\end{array}$ & $\begin{array}{l}-0.069 \\
(0.023)^{* *}\end{array}$ \\
\hline Lives alone & $\begin{array}{l}-6.989 \\
(7.187)\end{array}$ & $\begin{array}{l}-135.322 \\
(23.884)^{* *}\end{array}$ & $\begin{array}{l}0.117 \\
(0.026)^{* *}\end{array}$ & $\begin{array}{l}0.128 \\
(0.091)\end{array}$ & $\begin{array}{l}0.033 \\
(0.061)\end{array}$ & $\begin{array}{l}0.116 \\
(0.066)\end{array}$ & $\begin{array}{l}0.140 \\
(0.071)^{*}\end{array}$ & $\begin{array}{l}0.015 \\
(0.026)\end{array}$ \\
\hline household size & $\begin{array}{l}1.684 \\
(1.819)\end{array}$ & $\begin{array}{l}30.174 \\
(4.913)^{* *}\end{array}$ & $\begin{array}{l}-0.021 \\
(0.003)^{* *}\end{array}$ & $\begin{array}{l}0.045 \\
(0.013)^{* *}\end{array}$ & $\begin{array}{l}-0.022 \\
(0.008)^{* *}\end{array}$ & $\begin{array}{l}-0.027 \\
(0.008)^{* *}\end{array}$ & $\begin{array}{l}0.009 \\
(0.011)\end{array}$ & $\begin{array}{l}-0.002 \\
(0.004)\end{array}$ \\
\hline Incomplete primary & $\begin{array}{l}15.318 \\
(8.068)\end{array}$ & $\begin{array}{l}56.082 \\
(20.806)^{* *}\end{array}$ & $\begin{array}{l}-0.030 \\
(0.015)^{*}\end{array}$ & $\begin{array}{l}-0.233 \\
(0.056)^{* *}\end{array}$ & $\begin{array}{l}0.072 \\
(0.040)\end{array}$ & $\begin{array}{l}-0.089 \\
(0.040)^{*}\end{array}$ & $\begin{array}{l}-0.192 \\
(0.045)^{* *}\end{array}$ & $\begin{array}{l}-0.017 \\
(0.017)\end{array}$ \\
\hline Primary & $\begin{array}{l}61.025 \\
(23.866)^{*}\end{array}$ & $\begin{array}{l}258.457 \\
(53.409)^{* *}\end{array}$ & $\begin{array}{l}-0.051 \\
(0.022)^{*}\end{array}$ & $\begin{array}{l}-0.680 \\
(0.091)^{* *}\end{array}$ & $\begin{array}{l}0.058 \\
(0.057)\end{array}$ & $\begin{array}{l}-0.320 \\
(0.060)^{* *}\end{array}$ & $\begin{array}{l}-0.513 \\
(0.069)^{* *}\end{array}$ & $\begin{array}{l}-0.014 \\
(0.025)\end{array}$ \\
\hline Couple & 10.157 & 50.998 & -0.032 & 0.018 & -0.083 & 0.015 & 0.039 & 0.030 \\
\hline
\end{tabular}




\begin{tabular}{|c|c|c|c|c|c|c|c|c|}
\hline & $(10.301)$ & (30.864) & $(0.034)$ & $(0.121)$ & $(0.085)$ & $(0.090)$ & $(0.093)$ & $(0.024)$ \\
\hline \multirow[t]{2}{*}{ Divorced/Separated } & -4.787 & 53.830 & -0.012 & -0.124 & 0.023 & -0.073 & -0.054 & 0.088 \\
\hline & $(10.476)$ & $(40.843)$ & $(0.046)$ & $(0.175)$ & $(0.128)$ & $(0.125)$ & $(0.132)$ & $(0.056)$ \\
\hline \multirow[t]{2}{*}{ Widow } & -0.270 & 69.822 & -0.019 & -0.209 & -0.037 & -0.047 & -0.037 & 0.014 \\
\hline & (6.518) & $(26.973)^{* *}$ & $(0.033)$ & $(0.121)$ & $(0.083)$ & $(0.089)$ & $(0.092)$ & $(0.025)$ \\
\hline \multirow[t]{2}{*}{ Constant } & 43.372 & -336.349 & -1.232 & 4.160 & 1.460 & 2.706 & 4.846 & 0.624 \\
\hline & $(515.440)$ & $(1,392.582)$ & $(0.777)$ & (3.539) & (1.976) & (2.156) & (2.733) & $(0.663)$ \\
\hline Observations & 3929 & 3028 & 3621 & 4002 & 4000 & 4002 & 4002 & 4003 \\
\hline R-squared & 0.01 & 0.08 & 0.06 & 0.08 & 0.02 & 0.03 & 0.03 & 0.01 \\
\hline F-test education & 3.61 & 12.54 & 3.24 & 28.64 & 1.64 & 14.14 & 28.27 & 0.49 \\
\hline Prob $>F$ & 0.027 & 0.000 & 0.039 & 0.001 & 0.194 & 0.965 & 0.671 & 0.610 \\
\hline F-test age & 1.393 & 0.315 & 2.408 & 6.616 & 1.192 & 0.036 & 0.399 & 0.635 \\
\hline Prob $>F$ & 0.249 & 0.730 & 0.090 & 0.000 & 0.304 & 0.000 & 0.000 & 0.530 \\
\hline age parabola min/max & -2.264 & 78.511 & 84.908 & 265.935 & 163.678 & 100.926 & 81.855 & 87.726 \\
\hline
\end{tabular}


Table 4i: Treatment regressions

\begin{tabular}{|c|c|c|c|c|c|c|c|}
\hline & $\begin{array}{l}\text { Worked for } \\
\text { pay last } \\
\text { month (yes- } \\
\text { no [1-0]) }\end{array}$ & $\begin{array}{l}\text { Immediate } \\
\text { recall } \\
\text { (number of } \\
\text { words) }\end{array}$ & $\begin{array}{l}\text { Delayed } \\
\text { recall } \\
\text { (number of } \\
\text { words) }\end{array}$ & $\begin{array}{l}\text { Feel fear } \\
\text { someone } \\
\text { robbing you } \\
\text { (never, } \\
\text { sometimes, } \\
\text { usually, always } \\
{[1-4] \text { ) }}\end{array}$ & $\begin{array}{l}\text { Feel fear } \\
\text { someone close } \\
\text { to you will } \\
\text { take your } \\
\text { money (never, } \\
\text { sometimes, } \\
\text { usually, }\end{array}$ & $\begin{array}{l}\text { Money in a } \\
\text { safe place } \\
\text { (yes-no [1- } \\
0] \text { ) }\end{array}$ & $\begin{array}{l}\text { Feel verbally } \\
\text { or physically } \\
\text { abused (never, } \\
\text { sometimes, } \\
\text { usually, always } \\
{[1-4] \text { ) }}\end{array}$ \\
\hline Wave & $\begin{array}{l}0.002 \\
(0.020)\end{array}$ & $\begin{array}{l}-0.031 \\
(0.100)\end{array}$ & $\begin{array}{l}0.094 \\
(0.128)\end{array}$ & $\begin{array}{l}-0.186 \\
(0.060) * *\end{array}$ & $\begin{array}{l}-0.030 \\
(0.045)\end{array}$ & $\begin{array}{l}-0.006 \\
(0.013)\end{array}$ & $\begin{array}{l}-0.022 \\
(0.029)\end{array}$ \\
\hline Valladolid & $\begin{array}{l}-0.021 \\
(0.016)\end{array}$ & $\begin{array}{l}0.121 \\
(0.075)\end{array}$ & $\begin{array}{l}0.210 \\
(0.090)^{*}\end{array}$ & $\begin{array}{l}-0.152 \\
(0.043) * *\end{array}$ & $\begin{array}{l}-0.068 \\
(0.029)^{*}\end{array}$ & $\begin{array}{l}-0.015 \\
(0.008)^{*}\end{array}$ & $\begin{array}{l}-0.028 \\
(0.020)\end{array}$ \\
\hline Valladolid wave 2 & $\begin{array}{l}-0.043 \\
(0.016)^{* *}\end{array}$ & $\begin{array}{l}0.244 \\
(0.089) * *\end{array}$ & $\begin{array}{l}0.547 \\
(0.109) * *\end{array}$ & $\begin{array}{l}-0.005 \\
(0.053)\end{array}$ & $\begin{array}{l}0.004 \\
(0.037)\end{array}$ & $\begin{array}{l}-0.006 \\
(0.010)\end{array}$ & $\begin{array}{l}-0.017 \\
(0.028)\end{array}$ \\
\hline Age & $\begin{array}{l}-0.081 \\
(0.015)^{* *}\end{array}$ & $\begin{array}{l}-0.096 \\
(0.090)\end{array}$ & $\begin{array}{l}-0.241 \\
(0.100)^{*}\end{array}$ & $\begin{array}{l}-0.035 \\
(0.059)\end{array}$ & $\begin{array}{l}-0.011 \\
(0.044)\end{array}$ & $\begin{array}{l}0.009 \\
(0.008)\end{array}$ & $\begin{array}{l}-0.004 \\
(0.023)\end{array}$ \\
\hline Age squared & $\begin{array}{l}0.000 \\
(0.000)^{* *}\end{array}$ & $\begin{array}{l}0.000 \\
(0.001)\end{array}$ & $\begin{array}{l}0.001 \\
(0.001)\end{array}$ & $\begin{array}{l}0.000 \\
(0.000)\end{array}$ & $\begin{array}{l}0.000 \\
(0.000)\end{array}$ & $\begin{array}{l}-0.000 \\
(0.000)\end{array}$ & $\begin{array}{l}0.000 \\
(0.000)\end{array}$ \\
\hline Gender (male=1) & $\begin{array}{l}0.203 \\
(0.013)^{* *}\end{array}$ & $\begin{array}{l}-0.477 \\
(0.059) * *\end{array}$ & $\begin{array}{l}-0.545 \\
(0.070) * *\end{array}$ & $\begin{array}{l}-0.090 \\
(0.029) * *\end{array}$ & $\begin{array}{l}-0.045 \\
(0.021)^{*}\end{array}$ & $\begin{array}{l}-0.005 \\
(0.005)\end{array}$ & $\begin{array}{l}-0.053 \\
(0.017) * *\end{array}$ \\
\hline Speaks Maya & $\begin{array}{l}0.004 \\
(0.018)\end{array}$ & $\begin{array}{l}-0.306 \\
(0.084)^{* *}\end{array}$ & $\begin{array}{l}-0.198 \\
(0.106)\end{array}$ & $\begin{array}{l}-0.046 \\
(0.050)\end{array}$ & $\begin{array}{l}0.003 \\
(0.035)\end{array}$ & $\begin{array}{l}-0.008 \\
(0.011)\end{array}$ & $\begin{array}{l}-0.039 \\
(0.027)\end{array}$ \\
\hline Reads/writes Spanish & $\begin{array}{l}-0.022 \\
(0.015)\end{array}$ & $\begin{array}{l}0.445 \\
(0.078) * *\end{array}$ & $\begin{array}{l}0.415 \\
(0.091)^{* *}\end{array}$ & $\begin{array}{l}-0.061 \\
(0.048)\end{array}$ & $\begin{array}{l}0.016 \\
(0.033)\end{array}$ & $\begin{array}{l}0.007 \\
(0.009)\end{array}$ & $\begin{array}{l}0.011 \\
(0.024)\end{array}$ \\
\hline Lives alone & $\begin{array}{l}0.048 \\
(0.023)^{*}\end{array}$ & $\begin{array}{l}0.008 \\
(0.103)\end{array}$ & $\begin{array}{l}-0.133 \\
(0.121)\end{array}$ & $\begin{array}{l}0.028 \\
(0.055)\end{array}$ & $\begin{array}{l}0.058 \\
(0.038)\end{array}$ & $\begin{array}{l}0.009 \\
(0.011)\end{array}$ & $\begin{array}{l}0.070 \\
(0.029) *\end{array}$ \\
\hline household size & $\begin{array}{l}-0.000 \\
(0.003)\end{array}$ & $\begin{array}{l}-0.033 \\
(0.015)^{*}\end{array}$ & $\begin{array}{l}-0.032 \\
(0.018)\end{array}$ & $\begin{array}{l}-0.017 \\
(0.008) *\end{array}$ & $\begin{array}{l}-0.005 \\
(0.005)\end{array}$ & $\begin{array}{l}-0.001 \\
(0.001)\end{array}$ & $\begin{array}{l}0.007 \\
(0.005)\end{array}$ \\
\hline Incomplete primary & $\begin{array}{l}0.010 \\
(0.013)\end{array}$ & $\begin{array}{l}0.467 \\
(0.067)^{* *}\end{array}$ & $\begin{array}{l}0.527 \\
(0.080)^{* *}\end{array}$ & $\begin{array}{l}0.054 \\
(0.034)\end{array}$ & $\begin{array}{l}-0.001 \\
(0.023)\end{array}$ & $\begin{array}{l}0.002 \\
(0.006)\end{array}$ & $\begin{array}{l}0.018 \\
(0.019)\end{array}$ \\
\hline Primary & 0.015 & 1.152 & 1.167 & 0.043 & -0.075 & -0.009 & -0.039 \\
\hline
\end{tabular}




$\begin{array}{llllllll} & (0.024) & (0.116)^{* *} & (0.136)^{* *} & (0.052) & (0.032)^{*} & (0.008) & (0.022) \\ \text { Couple } & -0.050 & 0.082 & 0.008 & 0.023 & -0.020 & -0.017 & 0.041 \\ & (0.032) & (0.140) & (0.173) & (0.078) & (0.047) & (0.014) & (0.030) \\ \text { Divorced/Separated } & -0.014 & 0.100 & -0.167 & -0.039 & -0.073 & -0.030 & 0.144 \\ & (0.051) & (0.204) & (0.241) & (0.108) & (0.062) & (0.016) & (0.067)^{*} \\ \text { Widow } & -0.072 & -0.293 & -0.345 & -0.003 & -0.051 & -0.017 & 0.006 \\ & (0.032)^{*} & (0.138)^{*} & (0.169)^{*} & (0.078) & (0.047) & (0.014) & (0.030) \\ \text { Constant } & 3.804 & 8.607 & 14.677 & 3.085 & 1.717 & -0.316 & 1.304 \\ & (0.635)^{* *} & (3.662)^{*} & (4.071)^{* *} & (2.371) & (1.730) & (0.337) & (0.945) \\ \text { Observations } & 4006 & 4011 & 4011 & 3253 & 3251 & 3250 & 3253 \\ \text { R-squared } & 0.14 & 0.16 & 0.14 & 0.02 & 0.01 & 0.01 & 0.01 \\ \text { F-test education } & 0.38 & 55.04 & 43.36 & 1.24 & 3.70 & 1.41 & 4.33 \\ \text { Prob>F } & 0.686 & 0.000 & 0.000 & 0.288 & 0.318 & 0.245 & 0.013 \\ \text { F-test age } & 60.141 & 101.326 & 96.068 & 1.158 & 1.146 & 0.965 & 0.048 \\ \text { Prob>F } & 0.000 & 0.000 & 0.000 & 0.314 & 0.025 & 0.381 & 0.954 \\ \text { age parabola min/max } & 92.335 & 297.618 & 121.168 & 72.234 & 64.524 & 84.089 & 89.527\end{array}$

NOTE: We do not show in this table all the control variables included in the regressions. The reference categories for the control variables are wave 1, Motul, female, do not speak Maya, do not read write a message in Spanish, no schooling, single, and the missing category for respondent income and wealth. SOURCE: Baseline and first follow-up ENCAHEY, 2008 and 2009.

$* *=$ significant at $5 \%$ level of confidence. $*=$ significant at $10 \%$ level of confidence 
Table 5a: Interaction Effects

\begin{tabular}{|c|c|c|c|c|c|c|c|c|}
\hline & $\begin{array}{l}\text { Self-reported } \\
\text { health } \\
\text { (excellent, } \\
\text { very good, } \\
\text { good, fair, } \\
\text { poor [1-5]) }\end{array}$ & $\begin{array}{l}\text { Subjective } \\
\text { mortality } \\
\text { expectation } \\
\text { (chances to } \\
\text { leave at least } \\
10 \text { years more } \\
\text { [1-100 }\end{array}$ & $\begin{array}{l}\text { Feel sad, blue } \\
\text { or depressed } \\
\text { for } 2 \text { weeks } \\
\text { or more } \\
\text { during the } \\
\text { last } 3 \text { months } \\
\text { (yes-no }\end{array}$ & $\begin{array}{l}\text { Satisfied } \\
\text { relation fam. } \\
\text { members } \\
\text { (very } \\
\text { satisfied-very } \\
\text { unsatisfied } \\
\text { [1-5]) }\end{array}$ & $\begin{array}{l}\text { Satisfied hh } \\
\text { income (very } \\
\text { satisfied-very } \\
\text { unsatisfied } \\
{[1-5] \text { ) }}\end{array}$ & $\begin{array}{l}\text { Satisfied } \\
\text { social } \\
\text { contacts (very } \\
\text { satisfied-very } \\
\text { unsatisfied } \\
{[1-5] \text { ) }}\end{array}$ & $\begin{array}{l}\text { Satisfied with } \\
\text { job (very } \\
\text { satisfied-very } \\
\text { unsatisfied } \\
{[1-5] \text { ) }}\end{array}$ & $\begin{array}{l}\text { Satisfied with } \\
\text { health (very } \\
\text { satisfied-very } \\
\text { unsatisfied } \\
{[1-5] \text { ) }}\end{array}$ \\
\hline Wave & $\begin{array}{l}-0.230 \\
(0.045)^{* *}\end{array}$ & $\begin{array}{l}0.414 \\
(2.414)\end{array}$ & $\begin{array}{l}-0.203 \\
(0.037) * *\end{array}$ & $\begin{array}{l}-0.097 \\
(0.047)^{*}\end{array}$ & $\begin{array}{l}-0.185 \\
(0.060)^{* *}\end{array}$ & $\begin{array}{l}-0.068 \\
(0.051)\end{array}$ & $\begin{array}{l}-0.149 \\
(0.052)^{* *}\end{array}$ & $\begin{array}{l}-0.060 \\
(0.061)\end{array}$ \\
\hline Valladolid & $\begin{array}{l}0.088 \\
(0.032)^{* *}\end{array}$ & $\begin{array}{l}-4.375 \\
(1.577)^{* *}\end{array}$ & $\begin{array}{l}-0.080 \\
(0.026)^{* *}\end{array}$ & $\begin{array}{l}-0.009 \\
(0.034)\end{array}$ & $\begin{array}{l}0.023 \\
(0.044)\end{array}$ & $\begin{array}{l}-0.015 \\
(0.036)\end{array}$ & $\begin{array}{l}-0.022 \\
(0.039)\end{array}$ & $\begin{array}{l}0.024 \\
(0.045)\end{array}$ \\
\hline $\begin{array}{l}\text { Valladolid } \\
\text { wave } 2\end{array}$ & $\begin{array}{l}0.071 \\
(0.050)\end{array}$ & $\begin{array}{l}-2.242 \\
(2.916)\end{array}$ & (0.043) & $\begin{array}{l}0.020 \\
(0.053)\end{array}$ & $\begin{array}{l}-0.063 \\
(0.066)\end{array}$ & $(0.062)$ & $(0.062)$ & $\begin{array}{l}-0.106 \\
(0.069)\end{array}$ \\
\hline $\begin{array}{l}\text { Effect for } \\
\text { income } \\
\text { quartile } 1\end{array}$ & 0.068 & -1.592 & 0.005 & 0.121 & 0.067 & 0.088 & 0.017 & 0.040 \\
\hline $\begin{array}{l}\text { Effect for } \\
\text { income } \\
\text { quartile } 2\end{array}$ & 0.033 & -4.999 & 0.010 & -0.042 & 0.044 & -0.019 & -0.020 & 0.063 \\
\hline & $(0.072)$ & (3.623) & $(0.058)$ & $(0.074)$ & $(0.084)$ & $(0.071)$ & $(0.080)$ & $(0.086)$ \\
\hline $\begin{array}{l}\text { Effect for } \\
\text { income } \\
\text { quartile } 3\end{array}$ & 0.055 & 3.148 & -0.061 & -0.006 & 0.010 & -0.044 & -0.117 & $(0.085)$ \\
\hline $\begin{array}{l}\text { Effect for } \\
\text { income } \\
\text { quartile } 4\end{array}$ & 0.042 & -1.673 & 0.017 & 0.034 & 0.141 & 0.041 & -0.017 & 0.191 \\
\hline Effect on & $\begin{array}{l}(0.071) \\
-0.037\end{array}$ & $\begin{array}{l}(3.589) \\
0.616\end{array}$ & $\begin{array}{l}(0.058) \\
-0.020\end{array}$ & $\begin{array}{l}(0.071) \\
0.009\end{array}$ & $\begin{array}{l}(0.085) \\
-0.060\end{array}$ & $\begin{array}{l}(0.071) \\
-0.071\end{array}$ & $\begin{array}{l}(0.076) \\
-0.058\end{array}$ & $\begin{array}{l}(0.091)^{*} \\
-0.075\end{array}$ \\
\hline Constant & $\begin{array}{l}(0.047) \\
1.854\end{array}$ & $\begin{array}{l}(2.521) \\
308.603\end{array}$ & $\begin{array}{l}(0.038) \\
-0.817 \\
\end{array}$ & $\begin{array}{l}(0.048) \\
3.306\end{array}$ & $\begin{array}{l}(0.056) \\
6.013 \\
\end{array}$ & $\begin{array}{l}(0.048) \\
1.816\end{array}$ & $\begin{array}{l}(0.052) \\
2.677\end{array}$ & $\begin{array}{l}(0.058) \\
4.392\end{array}$ \\
\hline
\end{tabular}




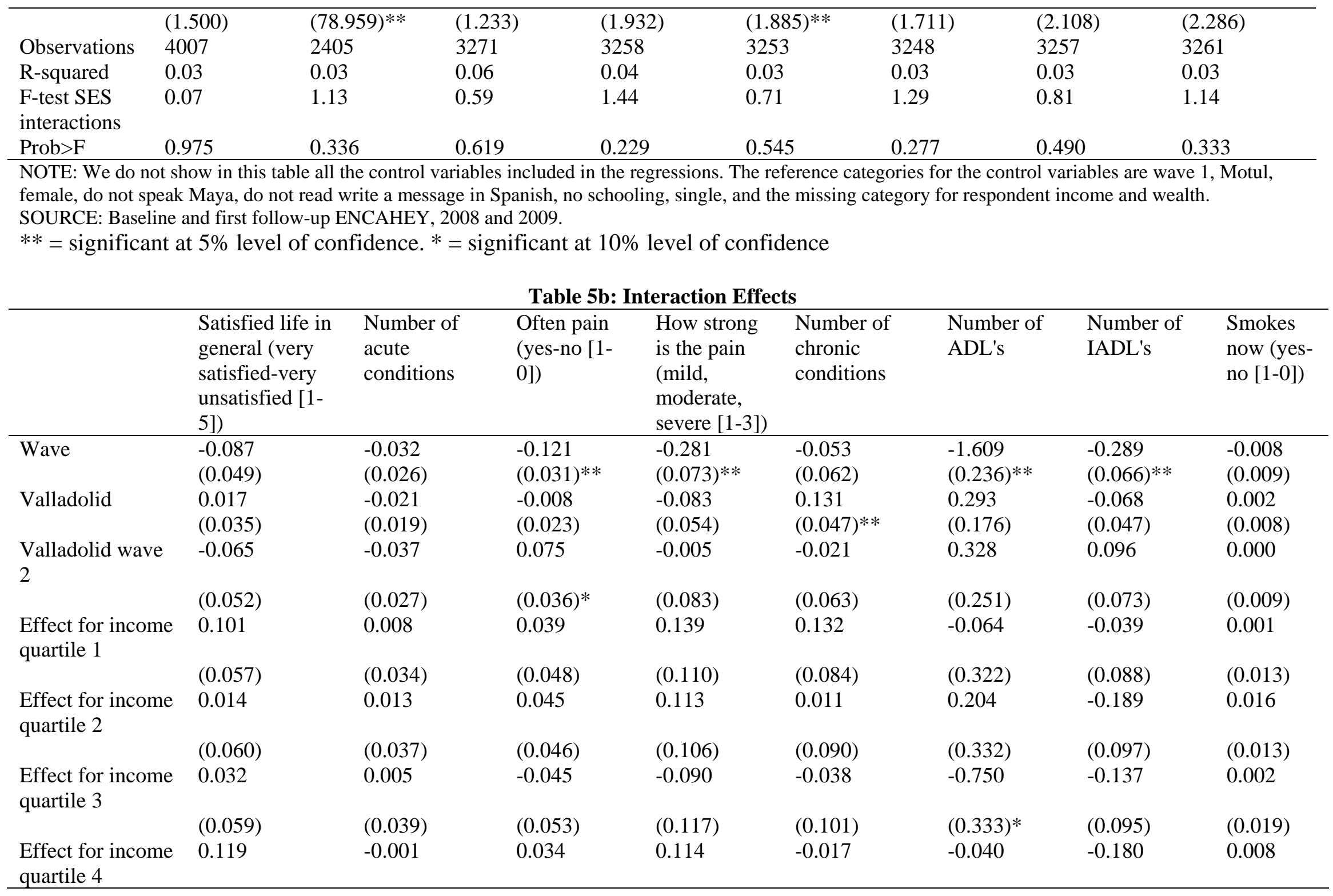




\begin{tabular}{|c|c|c|c|c|c|c|c|c|}
\hline & $(0.064)$ & $(0.040)$ & $(0.050)$ & (0.113) & $(0.095)$ & $(0.341)$ & $(0.089)^{*}$ & $(0.019)$ \\
\hline \multirow[t]{2}{*}{ Effect on males } & -0.050 & -0.006 & -0.042 & -0.069 & 0.065 & -0.232 & 0.009 & -0.001 \\
\hline & $(0.042)$ & $(0.025)$ & $(0.033)$ & $(0.075)$ & $(0.061)$ & $(0.231)$ & $(0.064)$ & $(0.011)$ \\
\hline \multirow[t]{2}{*}{ Constant } & 1.441 & -1.894 & -0.670 & -2.642 & -6.746 & -13.168 & -1.436 & 0.326 \\
\hline & (1.558) & $(0.684)^{* *}$ & (0.996) & $(2.235)$ & $(1.965)^{* *}$ & $(8.725)$ & $(2.331)$ & $(0.367)$ \\
\hline Observations & 3257 & 4011 & 4006 & 4003 & 4011 & 3769 & 4002 & 4008 \\
\hline R-squared & 0.03 & 0.02 & 0.03 & 0.03 & 0.03 & 0.07 & 0.06 & 0.03 \\
\hline $\begin{array}{l}\text { F-test SES } \\
\text { interactions }\end{array}$ & 1.10 & 0.03 & 0.96 & 1.24 & 1.03 & 2.32 & 0.92 & 0.35 \\
\hline Prob $>F$ & 0.347 & 0.993 & 0.410 & 0.294 & 0.377 & 0.074 & 0.433 & 0.787 \\
\hline
\end{tabular}

NOTE: We do not show in this table all the control variables included in the regressions. The reference categories for the control variables are wave 1, Motul, female, do not speak Maya, do not read write a message in Spanish, no schooling, single, and the missing category for respondent income and wealth. SOURCE: Baseline and first follow-up ENCAHEY, 2008 and 2009.

$* *=$ significant at $5 \%$ level of confidence. $*=$ significant at $10 \%$ level of confidence

Table 5c: Interaction Effects

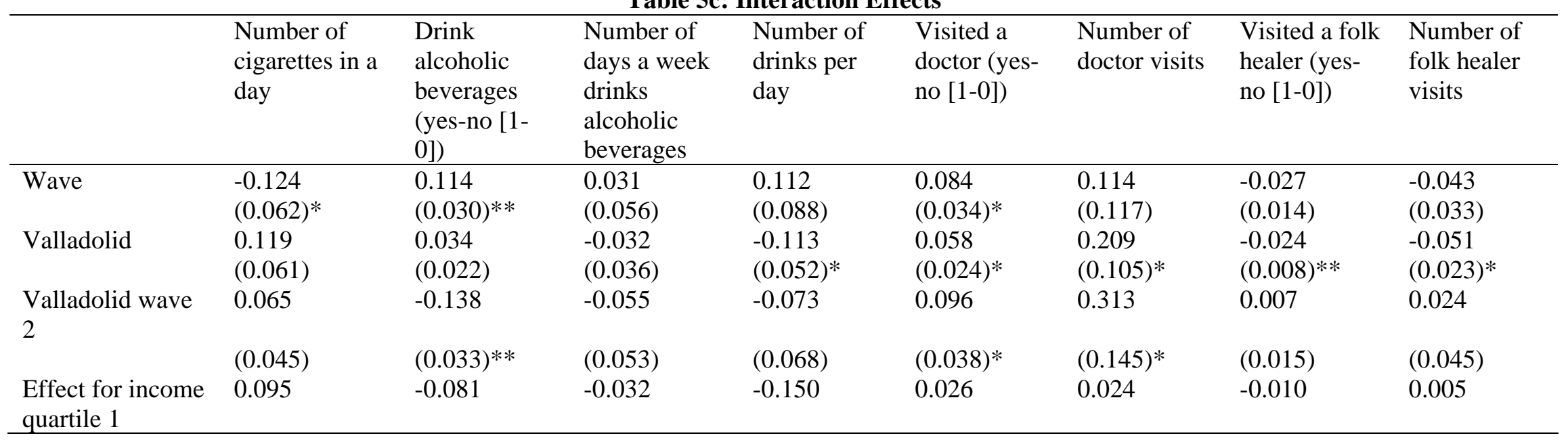




\begin{tabular}{|c|c|c|c|c|c|c|c|c|}
\hline & $(0.058)$ & $(0.042)$ & $(0.060)$ & $(0.091)$ & $(0.047)$ & $(0.188)$ & $(0.018)$ & $(0.052)$ \\
\hline \multirow{2}{*}{$\begin{array}{l}\text { Effect for income } \\
\text { quartile } 2\end{array}$} & 0.110 & -0.038 & -0.004 & 0.048 & -0.041 & -0.102 & -0.020 & -0.024 \\
\hline & $(0.055)^{*}$ & $(0.043)$ & $(0.054)$ & $(0.114)$ & $(0.049)$ & $(0.183)$ & $(0.019)$ & $(0.048)$ \\
\hline \multirow{2}{*}{$\begin{array}{l}\text { Effect for income } \\
\text { quartile } 3\end{array}$} & 0.022 & -0.037 & 0.013 & -0.071 & -0.122 & -0.284 & -0.024 & -0.054 \\
\hline & $(0.079)$ & $(0.047)$ & $(0.063)$ & $(0.089)$ & $(0.055)^{*}$ & $(0.227)$ & $(0.021)$ & $(0.048)$ \\
\hline \multirow{2}{*}{$\begin{array}{l}\text { Effect for income } \\
\text { quartile } 4\end{array}$} & 0.082 & -0.051 & 0.015 & -0.093 & -0.004 & -0.129 & -0.039 & -0.053 \\
\hline & $(0.166)$ & $(0.046)$ & (0.098) & $(0.158)$ & $(0.054)$ & $(0.236)$ & $(0.020)^{*}$ & $(0.050)$ \\
\hline \multirow[t]{2}{*}{ Effect on males } & -0.099 & 0.023 & -0.037 & -0.044 & 0.010 & 0.070 & -0.013 & -0.085 \\
\hline & $(0.060)$ & (0.029) & $(0.048)$ & $(0.075)$ & $(0.034)$ & (0.143) & $(0.014)$ & $(0.034)^{*}$ \\
\hline \multirow[t]{2}{*}{ Constant } & -1.107 & 0.674 & 2.279 & 4.230 & -0.406 & -4.540 & -0.296 & -1.445 \\
\hline & (1.668) & $(0.941)$ & (1.331) & $(2.260)$ & $(1.224)$ & (3.963) & $(0.310)$ & $(0.725)^{*}$ \\
\hline Observations & 4011 & 4010 & 3983 & 3992 & 4008 & 4004 & 4009 & 4009 \\
\hline R-squared & 0.03 & 0.20 & 0.04 & 0.05 & 0.04 & 0.03 & 0.02 & 0.01 \\
\hline $\begin{array}{l}\text { F-test SES } \\
\text { interactions }\end{array}$ & 0.67 & 0.34 & 0.16 & 0.77 & 2.13 & 0.81 & 0.58 & 0.54 \\
\hline Prob $>F$ & 0.568 & 0.797 & 0.926 & 0.509 & 0.095 & 0.489 & 0.630 & 0.655 \\
\hline
\end{tabular}

NOTE: We do not show in this table all the control variables included in the regressions. The reference categories for the control variables are wave 1, Motul, female, do not speak Maya, do not read write a message in Spanish, no schooling, single, and the missing category for respondent income and wealth. SOURCE: Baseline and first follow-up ENCAHEY, 2008 and 2009.

$* *=$ significant at $5 \%$ level of confidence. $*=$ significant at $10 \%$ level of confidence

Table 5d: Interaction Effects

\begin{tabular}{|c|c|c|c|c|c|c|c|}
\hline & \multicolumn{7}{|c|}{ 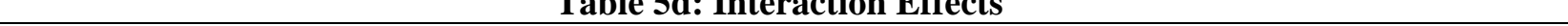 } \\
\hline & $\begin{array}{l}\text { Visited a } \\
\text { dentist } \\
\text { (yes-no [1- } \\
0] \text { ) }\end{array}$ & $\begin{array}{l}\text { Number of } \\
\text { dentist } \\
\text { visits }\end{array}$ & $\begin{array}{l}\text { Outpatient } \\
\text { procedures } \\
\text { (yes-no [1-0]) }\end{array}$ & $\begin{array}{l}\text { Consulted a } \\
\text { pharmacist } \\
\text { (yes-no [1-0]) }\end{array}$ & $\begin{array}{l}\text { Bought no } \\
\text { medicines } \\
\text { because they } \\
\text { are too } \\
\text { expensive } \\
\text { (yes-no [1- } \\
0] \text { ) }\end{array}$ & $\begin{array}{l}\text { Pay out-of- } \\
\text { pocket (oop) } \\
\text { medical cost } \\
\text { or medication } \\
\text { (yes-no [1-0]) }\end{array}$ & $\begin{array}{l}\text { Oop } \\
\text { expenses } \\
\text { paid by } \\
\text { relatives } \\
\text { (yes-no [1- } \\
0] \text { ) }\end{array}$ \\
\hline Wave & $\begin{array}{l}0.025 \\
(0.017)\end{array}$ & $\begin{array}{l}0.043 \\
(0.048)\end{array}$ & $\begin{array}{l}-0.002 \\
(0.007)\end{array}$ & $\begin{array}{l}-0.085 \\
(0.019)^{* *}\end{array}$ & $\begin{array}{l}-0.146 \\
(0.029)^{* *}\end{array}$ & $\begin{array}{l}-0.047 \\
(0.035)\end{array}$ & $\begin{array}{l}-0.019 \\
(0.029)\end{array}$ \\
\hline
\end{tabular}




\begin{tabular}{|c|c|c|c|c|c|c|c|}
\hline Valladolid & $\begin{array}{l}-0.017 \\
(0.012)\end{array}$ & $\begin{array}{l}-0.061 \\
(0.034)\end{array}$ & $\begin{array}{l}-0.002 \\
(0.005)\end{array}$ & $\begin{array}{l}-0.025 \\
(0.011)^{*}\end{array}$ & $\begin{array}{l}-0.042 \\
(0.019)^{*}\end{array}$ & $\begin{array}{l}-0.046 \\
(0.024)\end{array}$ & $\begin{array}{l}-0.095 \\
(0.019) * *\end{array}$ \\
\hline \multirow{2}{*}{$\begin{array}{l}\text { Valladolid wave } \\
2\end{array}$} & -0.030 & -0.067 & 0.005 & 0.026 & -0.027 & 0.062 & -0.110 \\
\hline & (0.019) & $(0.050)$ & $(0.007)$ & $(0.019)$ & $(0.029)$ & (0.039) & $(0.030)^{* *}$ \\
\hline \multirow{2}{*}{$\begin{array}{l}\text { Effect for income } \\
\text { quartile } 1\end{array}$} & 0.011 & 0.040 & -0.014 & -0.005 & -0.030 & -0.063 & -0.012 \\
\hline & $(0.020)$ & $(0.048)$ & (0.009) & $(0.025)$ & $(0.037)$ & (0.049) & $(0.039)$ \\
\hline \multirow{2}{*}{$\begin{array}{l}\text { Effect for income } \\
\text { quartile } 2\end{array}$} & 0.030 & 0.073 & -0.015 & 0.005 & 0.009 & -0.085 & -0.033 \\
\hline & $(0.024)$ & $(0.051)$ & (0.009) & $(0.029)$ & $(0.038)$ & $(0.049)$ & $(0.036)$ \\
\hline \multirow{2}{*}{$\begin{array}{l}\text { Effect for income } \\
\text { quartile } 3\end{array}$} & 0.049 & 0.063 & -0.016 & -0.059 & 0.010 & -0.138 & -0.030 \\
\hline & (0.029) & $(0.058)$ & $(0.010)$ & $(0.019) * *$ & $(0.038)$ & $(0.054)^{*}$ & $(0.035)$ \\
\hline \multirow{2}{*}{$\begin{array}{l}\text { Effect for income } \\
\text { quartile } 4\end{array}$} & 0.034 & 0.120 & -0.007 & -0.035 & 0.026 & -0.020 & -0.011 \\
\hline & (0.033) & $(0.091)$ & (0.009) & (0.019) & $(0.034)$ & $(0.052)$ & $(0.035)$ \\
\hline Effect on males & $\begin{array}{l}-0.010 \\
(0.017)\end{array}$ & $\begin{array}{l}-0.077 \\
(0.042)\end{array}$ & $\begin{array}{l}-0.001 \\
(0.006)\end{array}$ & $\begin{array}{l}-0.021 \\
(0.016)\end{array}$ & $\begin{array}{l}-0.057 \\
(0.023) *\end{array}$ & $\begin{array}{l}-0.024 \\
(0.033)\end{array}$ & $\begin{array}{l}0.027 \\
(0.024)\end{array}$ \\
\hline Constant & $\begin{array}{l}0.695 \\
(0.448)\end{array}$ & $\begin{array}{l}1.516 \\
(1.196)\end{array}$ & $\begin{array}{l}0.132 \\
(0.215)\end{array}$ & $\begin{array}{l}-0.027 \\
(0.426)\end{array}$ & $\begin{array}{l}-0.390 \\
(0.699)\end{array}$ & $\begin{array}{l}0.516 \\
(1.098)\end{array}$ & $\begin{array}{l}0.494 \\
(0.947)\end{array}$ \\
\hline Observations & 4008 & 4006 & 4008 & 4003 & 4006 & 4007 & 4006 \\
\hline R-squared & 0.02 & 0.02 & 0.01 & 0.03 & 0.04 & 0.02 & 0.07 \\
\hline $\begin{array}{l}\text { F-test SES } \\
\text { interactions }\end{array}$ & 0.56 & 0.31 & 0.26 & 2.41 & 0.64 & 1.21 & 0.17 \\
\hline Prob $>F$ & 0.644 & 0.819 & 0.858 & 0.065 & 0.588 & 0.305 & 0.915 \\
\hline
\end{tabular}

NOTE: We do not show in this table all the control variables included in the regressions. The reference categories for the control variables are wave 1, Motul, female, do not speak Maya, do not read write a message in Spanish, no schooling, single, and the missing category for respondent income and wealth. The model for the number of outpatient procedures could not be estimated due to the few numbers of observations.

SOURCE: Baseline and first follow-up ENCAHEY, 2008 and 2009.

** = significant at $5 \%$ level of confidence. $*=$ significant at $10 \%$ level of confidence 
Table 5e: Interaction Effects

\begin{tabular}{|c|c|c|c|c|c|c|c|c|}
\hline & $\begin{array}{l}\text { Oop } \\
\text { expenses } \\
\text { paid by } \\
\text { elderly } \\
\text { eligible (yes- } \\
\text { no [1-0]) }\end{array}$ & $\begin{array}{l}\text { Serious } \\
\text { health } \\
\text { problem but } \\
\text { did not go to } \\
\text { the doctor } \\
\text { (yes-no[1-0]) }\end{array}$ & $\begin{array}{l}\text { Did not go to } \\
\text { the doctor } \\
\text { because of } \\
\text { money (yes- } \\
\text { no[1-0]) }\end{array}$ & $\begin{array}{l}\text { Relatives or } \\
\text { friends pay } \\
\text { your } \\
\text { expenses (not } \\
\text { true, } \\
\text { sometimes } \\
\text { true, often } \\
\text { true, al }\end{array}$ & $\begin{array}{l}\text { Feel a burden } \\
\text { on your } \\
\text { family or } \\
\text { friends (not } \\
\text { true, } \\
\text { sometimes } \\
\text { true, often } \\
\text { true, a }\end{array}$ & $\begin{array}{l}\text { Activities you } \\
\text { used to do but } \\
\text { can't do } \\
\text { because of } \\
\text { lack of money } \\
\text { (yes-no[1-0]) }\end{array}$ & $\begin{array}{l}\text { Donation to } \\
\text { the church } \\
\text { but can't do } \\
\text { due to } \\
\text { money (yes- } \\
\text { no[1-0]) }\end{array}$ & $\begin{array}{l}\text { Donation to } \\
\text { family or } \\
\text { friends } \\
\text { parties but } \\
\text { can't do due } \\
\text { to money } \\
\text { (yes-no[1- } \\
0] \text { ) }\end{array}$ \\
\hline \multirow[t]{2}{*}{ Wave } & -0.034 & -0.106 & -0.088 & 0.094 & -0.344 & -0.143 & -0.015 & -0.005 \\
\hline & $(0.028)$ & $(0.024)^{* *}$ & $(0.020) * *$ & $(0.078)$ & $(0.151)^{*}$ & $(0.036) * *$ & $(0.008)$ & $(0.008)$ \\
\hline \multirow[t]{2}{*}{ Valladolid } & 0.042 & -0.049 & -0.020 & -0.071 & 0.101 & -0.098 & -0.005 & 0.000 \\
\hline & $(0.019) *$ & $(0.017)^{* *}$ & $(0.014)$ & $(0.057)$ & $(0.106)$ & $(0.025) * *$ & $(0.006)$ & $(0.007)$ \\
\hline \multirow{2}{*}{$\begin{array}{l}\text { Valladolid wave } \\
2\end{array}$} & 0.121 & -0.023 & 0.000 & -0.135 & 0.355 & -0.039 & -0.004 & 0.011 \\
\hline & $(0.032)^{* *}$ & $(0.025)$ & $(0.020)$ & $(0.093)$ & $(0.198)$ & $(0.040)$ & $(0.010)$ & $(0.011)$ \\
\hline \multirow{2}{*}{$\begin{array}{l}\text { Effect for income } \\
\text { quartile } 1\end{array}$} & -0.028 & -0.023 & -0.038 & 0.091 & 0.167 & -0.012 & 0.015 & -0.022 \\
\hline & $(0.040)$ & $(0.029)$ & $(0.023)$ & $(0.124)$ & $(0.243)$ & $(0.049)$ & $(0.014)$ & $(0.012)$ \\
\hline \multirow{2}{*}{$\begin{array}{l}\text { Effect for income } \\
\text { quartile } 2\end{array}$} & -0.034 & -0.014 & -0.033 & 0.167 & -0.035 & 0.041 & & -0.003 \\
\hline & $(0.042)$ & $(0.033)$ & $(0.025)$ & $(0.125)$ & $(0.260)$ & $(0.051)$ & & $(0.013)$ \\
\hline \multirow{2}{*}{$\begin{array}{l}\text { Effect for income } \\
\text { quartile } 3\end{array}$} & -0.066 & -0.023 & -0.023 & 0.302 & 0.031 & 0.029 & 0.002 & -0.022 \\
\hline & $(0.047)$ & $(0.032)$ & $(0.024)$ & $(0.132)^{*}$ & $(0.276)$ & $(0.052)$ & $(0.014)$ & $(0.013)$ \\
\hline \multirow{2}{*}{$\begin{array}{l}\text { Effect for income } \\
\text { quartile } 4\end{array}$} & 0.014 & 0.018 & 0.031 & 0.075 & 0.064 & 0.061 & 0.016 & -0.010 \\
\hline & $(0.047)$ & $(0.030)$ & $(0.021)$ & $(0.121)$ & $(0.282)$ & $(0.047)$ & $(0.011)$ & $(0.011)$ \\
\hline \multirow[t]{2}{*}{ Effect on males } & -0.037 & 0.011 & -0.008 & 0.020 & -0.248 & 0.003 & -0.002 & 0.002 \\
\hline & $(0.028)$ & $(0.020)$ & $(0.015)$ & $(0.078)$ & $(0.174)$ & $(0.033)$ & (0.008) & (0.009) \\
\hline \multirow[t]{2}{*}{ Constant } & 0.001 & 0.373 & 0.439 & -1.675 & 0.371 & 2.406 & 0.126 & 0.233 \\
\hline & (0.712) & $(0.552)$ & $(0.476)$ & $(2.726)$ & $(4.826)$ & $(1.019)^{*}$ & $(0.202)$ & $(0.224)$ \\
\hline Observations & 4006 & 3999 & 4011 & 3996 & 910 & 3254 & 4011 & 4011 \\
\hline
\end{tabular}




\begin{tabular}{|c|c|c|c|c|c|c|c|c|}
\hline R-squared & 0.04 & 0.03 & 0.03 & 0.11 & 0.04 & 0.05 & 0.01 & 0.01 \\
\hline $\begin{array}{l}\text { F-test SES } \\
\text { interactions }\end{array}$ & 0.64 & 0.68 & 4.04 & 1.02 & 0.18 & 0.58 & 0.52 & 0.78 \\
\hline Prob $>F$ & 0.586 & 0.566 & 0.007 & 0.381 & 0.913 & 0.628 & 0.594 & 0.506 \\
\hline
\end{tabular}

NOTE: We do not show in this table all the control variables included in the regressions. The reference categories for the control variables are wave 1, Motul, female, do not speak Maya, do not read write a message in Spanish, no schooling, single, and the missing category for respondent income and wealth. SOURCE: Baseline and first follow-up ENCAHEY, 2008 and 2009.

$* *=$ significant at $5 \%$ level of confidence. * = significant at $10 \%$ level of confidence

Table 5f: Interaction Effects

\begin{tabular}{|c|c|c|c|c|c|c|c|c|}
\hline & $\begin{array}{l}\text { Community } \\
\text { activities but } \\
\text { can't do due } \\
\text { to money } \\
\text { (yes-no[1-0]) }\end{array}$ & $\begin{array}{l}\text { Help out } \\
\text { family } \\
\text { members but } \\
\text { can't do due } \\
\text { to money } \\
\text { (yes-no[1-0]) }\end{array}$ & $\begin{array}{l}\text { Help out non- } \\
\text { relatives but } \\
\text { can't do due } \\
\text { to money } \\
\text { (yes-no[1-0]) }\end{array}$ & $\begin{array}{l}\text { Travel to visit } \\
\text { family or } \\
\text { friends but } \\
\text { can't do due } \\
\text { to money } \\
\text { (yes-no[1-0]) }\end{array}$ & $\begin{array}{l}\text { Sometimes } \\
\text { do not have } \\
\text { enough to eat } \\
\text { (yes-no[1-0]) }\end{array}$ & $\begin{array}{l}\text { Often we do } \\
\text { not have } \\
\text { enough to eat } \\
\text { (yes-no[1-0]) }\end{array}$ & $\begin{array}{l}\text { Sometimes or } \\
\text { often we } \\
\text { don't have } \\
\text { enough to eat } \\
\text { because of } \\
\text { money (yes- } \\
\text { no[1-0]) }\end{array}$ & $\begin{array}{l}\text { Often worried } \\
\text { to run out of } \\
\text { food last } \\
\text { three months } \\
\text { (never-always } \\
\text { [1-4]) }\end{array}$ \\
\hline \multirow[t]{2}{*}{ Wave } & -0.002 & -0.030 & -0.034 & -0.023 & -0.163 & -0.050 & 0.040 & -0.411 \\
\hline & $(0.007)$ & $(0.023)$ & $(0.012)^{* *}$ & $(0.014)$ & $(0.035) * *$ & $(0.019)^{* *}$ & (0.033) & $(0.069)^{* *}$ \\
\hline \multirow[t]{2}{*}{ Valladolid } & -0.015 & -0.019 & -0.012 & -0.027 & 0.058 & 0.023 & 0.104 & -0.014 \\
\hline & $(0.005)^{* *}$ & $(0.016)$ & $(0.007)$ & $(0.010) * *$ & $(0.025) *$ & $(0.013)$ & $(0.025)^{* *}$ & $(0.049)$ \\
\hline \multirow{2}{*}{$\begin{array}{l}\text { Valladolid } \\
\text { wave } 2\end{array}$} & -0.010 & -0.013 & -0.006 & -0.010 & 0.022 & 0.043 & 0.056 & 0.198 \\
\hline & $(0.008)$ & $(0.025)$ & (0.009) & $(0.014)$ & $(0.038)$ & $(0.019)^{*}$ & $(0.038)$ & $(0.080)^{*}$ \\
\hline \multirow{2}{*}{$\begin{array}{l}\text { Effect for } \\
\text { income } \\
\text { quartile } 1\end{array}$} & 0.000 & 0.052 & -0.019 & -0.031 & 0.000 & -0.041 & -0.007 & -0.071 \\
\hline & $(0.008)$ & $(0.033)$ & $(0.012)$ & $(0.018)$ & $(0.045)$ & $(0.026)$ & $(0.046)$ & $(0.102)$ \\
\hline \multirow{2}{*}{$\begin{array}{l}\text { Effect for } \\
\text { income } \\
\text { quartile } 2\end{array}$} & 0.013 & 0.031 & 0.018 & -0.007 & -0.015 & -0.007 & 0.026 & -0.019 \\
\hline & $(0.012)$ & $(0.035)$ & $(0.016)$ & $(0.016)$ & $(0.047)$ & $(0.025)$ & $(0.048)$ & (0.105) \\
\hline Effect for & -0.014 & 0.065 & 0.006 & 0.001 & 0.009 & 0.005 & 0.037 & -0.045 \\
\hline
\end{tabular}




\begin{tabular}{|c|c|c|c|c|c|c|c|c|}
\hline \multicolumn{9}{|l|}{$\begin{array}{l}\text { income } \\
\text { quartile } 3\end{array}$} \\
\hline & $(0.012)$ & (0.039) & $(0.013)$ & $(0.015)$ & $(0.051)$ & $(0.023)$ & $(0.053)$ & $(0.105)$ \\
\hline \multirow{2}{*}{$\begin{array}{l}\text { Effect for } \\
\text { income } \\
\text { quartile } 4\end{array}$} & 0.010 & 0.084 & 0.006 & 0.010 & -0.011 & -0.003 & 0.006 & -0.146 \\
\hline & $(0.011)$ & $(0.036) *$ & (0.009) & $(0.017)$ & $(0.042)$ & $(0.020)$ & $(0.043)$ & $(0.090)$ \\
\hline \multirow{2}{*}{$\begin{array}{l}\text { Effect on } \\
\text { males }\end{array}$} & -0.001 & 0.003 & 0.000 & -0.010 & -0.066 & -0.019 & -0.067 & -0.087 \\
\hline & $(0.008)$ & $(0.024)$ & $(0.009)$ & $(0.010)$ & $(0.025)^{* *}$ & $(0.013)$ & $(0.026)^{*}$ & $(0.054)$ \\
\hline \multirow[t]{2}{*}{ Constant } & 0.178 & 0.932 & 0.043 & -0.072 & 0.315 & 0.047 & 0.410 & 5.577 \\
\hline & $(0.173)$ & $(0.637)$ & $(0.230)$ & $(0.314)$ & (1.091) & $(0.429)$ & (1.084) & $(2.471)^{*}$ \\
\hline Observations & 4011 & 4011 & 4011 & 4011 & 4011 & 4011 & 4011 & 3624 \\
\hline R-squared & 0.01 & 0.02 & 0.02 & 0.02 & 0.05 & 0.02 & 0.04 & 0.05 \\
\hline $\begin{array}{l}\text { F-test SES } \\
\text { interactions }\end{array}$ & 1.44 & 0.54 & 1.82 & 1.44 & 0.08 & 0.96 & 0.24 & 0.59 \\
\hline Prob $>F$ & 0.229 & 0.656 & 0.141 & 0.230 & 0.973 & 0.413 & 0.869 & 0.620 \\
\hline
\end{tabular}

NOTE: We do not show in this table all the control variables included in the regressions. The reference categories for the control variables are wave 1, Motul, female, do not speak Maya, do not read write a message in Spanish, no schooling, single, and the missing category for respondent income and wealth. SOURCE: Baseline and first follow-up ENCAHEY, 2008 and 2009.

$* *=$ significant at $5 \%$ level of confidence. $*=$ significant at $10 \%$ level of confidence 
Table 5g: Interaction Effects

\begin{tabular}{|c|c|c|c|c|c|c|c|c|}
\hline & $\begin{array}{l}\text { Run out of } \\
\text { food and } \\
\text { money not } \\
\text { enough } \\
\text { (never-always } \\
{[1-4] \text { ) }}\end{array}$ & $\begin{array}{l}\text { Skip or cut } \\
\text { meals (never- } \\
\text { always [1-4]) }\end{array}$ & $\begin{array}{l}\text { Often eat less } \\
\text { than you felt } \\
\text { you should } \\
\text { (never-always } \\
{[1-4] \text { ) }}\end{array}$ & $\begin{array}{l}\text { Often hungry } \\
\text { (never-always } \\
{[1-4] \text { ) }}\end{array}$ & $\begin{array}{l}\text { Not eat all } \\
\text { day (never- } \\
\text { always [1-4]) }\end{array}$ & $\begin{array}{l}\text { Food from } \\
\text { charity } \\
\text { (never-always } \\
{[1-4] \text { ) }}\end{array}$ & $\begin{array}{l}\text { Spend on } \\
\text { food at home } \\
\text { last week }\end{array}$ & $\begin{array}{l}\text { Spend on } \\
\text { food away } \\
\text { from home }\end{array}$ \\
\hline \multirow[t]{2}{*}{ Wave } & -0.253 & -0.301 & -0.237 & -0.258 & -0.161 & -0.052 & 38.436 & 5.754 \\
\hline & $(0.059) * *$ & $(0.058)^{* *}$ & $(0.054)^{* *}$ & $(0.049) * *$ & $(0.039) * *$ & $(0.025) *$ & (21.788) & (9.163) \\
\hline \multirow[t]{2}{*}{ Valladolid } & -0.129 & -0.074 & -0.067 & -0.093 & -0.077 & -0.037 & -24.156 & -10.400 \\
\hline & $(0.041)^{* *}$ & $(0.041)$ & $(0.038)$ & $(0.033)^{* *}$ & $(0.024)^{* *}$ & $(0.015) *$ & (17.506) & (8.841) \\
\hline \multirow{2}{*}{$\begin{array}{l}\text { Valladolid } \\
\text { wave } 2\end{array}$} & -0.031 & 0.018 & -0.039 & -0.052 & -0.096 & -0.048 & -6.333 & -5.692 \\
\hline & $(0.065)$ & $(0.064)$ & $(0.059)$ & $(0.047)$ & $(0.034)^{* *}$ & $(0.019) *$ & (26.385) & (22.701) \\
\hline \multirow{2}{*}{$\begin{array}{l}\text { Effect for } \\
\text { income } \\
\text { quartile } 1\end{array}$} & -0.083 & -0.086 & -0.083 & -0.082 & -0.024 & 0.010 & -3.953 & -5.986 \\
\hline & $(0.091)$ & $(0.086)$ & $(0.077)$ & $(0.061)$ & $(0.046)$ & $(0.029)$ & (28.952) & (20.445) \\
\hline \multirow{2}{*}{$\begin{array}{l}\text { Effect for } \\
\text { income } \\
\text { quartile } 2\end{array}$} & -0.044 & -0.026 & -0.004 & 0.085 & 0.064 & -0.018 & -0.198 & -14.132 \\
\hline & $(0.087)$ & $(0.080)$ & $(0.080)$ & $(0.061)$ & $(0.038)$ & $(0.036)$ & (32.994) & (19.129) \\
\hline \multirow{2}{*}{$\begin{array}{l}\text { Effect for } \\
\text { income } \\
\text { quartile } 3\end{array}$} & -0.023 & -0.091 & -0.003 & 0.024 & 0.013 & 0.016 & 1.204 & -16.355 \\
\hline & $(0.090)$ & $(0.084)$ & $(0.077)$ & $(0.055)$ & $(0.040)$ & $(0.029)$ & (31.481) & $(20.423)$ \\
\hline \multirow{2}{*}{$\begin{array}{l}\text { Effect for } \\
\text { income } \\
\text { quartile } 4\end{array}$} & -0.070 & -0.111 & -0.029 & 0.082 & 0.037 & 0.009 & 20.384 & 5.000 \\
\hline & $(0.074)$ & $(0.070)$ & $(0.065)$ & $(0.045)$ & $(0.032)$ & $(0.024)$ & (41.748) & (28.950) \\
\hline \multirow{2}{*}{$\begin{array}{l}\text { Effect on } \\
\text { males }\end{array}$} & -0.166 & -0.093 & -0.091 & -0.124 & -0.042 & -0.011 & 44.151 & -2.530 \\
\hline & $(0.046)^{* *}$ & $(0.046)^{*}$ & $(0.042)^{*}$ & $(0.032)^{* *}$ & $(0.023)$ & $(0.017)$ & $(19.261)^{*}$ & (6.556) \\
\hline \multirow[t]{2}{*}{ Constant } & 6.510 & 4.145 & 6.613 & 5.564 & 3.252 & 1.182 & -229.883 & 45.294 \\
\hline & $(2.052)^{* *}$ & $(2.064)^{*}$ & $(1.746)^{* *}$ & $(1.653)^{* *}$ & $(1.286)^{*}$ & $(0.666)$ & (935.847) & $(538.746)$ \\
\hline
\end{tabular}




\begin{tabular}{|c|c|c|c|c|c|c|c|c|}
\hline Observations & 3624 & 3619 & 3623 & 3624 & 3625 & 3630 & 3041 & 3929 \\
\hline R-squared & 0.08 & 0.06 & 0.06 & 0.08 & 0.07 & 0.02 & 0.16 & 0.02 \\
\hline $\begin{array}{l}\text { F-test SES } \\
\text { interactions }\end{array}$ & 0.16 & 0.36 & 0.36 & 2.62 & 1.17 & 0.25 & 0.09 & 0.28 \\
\hline Prob $>F$ & 0.926 & 0.779 & 0.783 & 0.049 & 0.321 & 0.863 & 0.964 & 0.839 \\
\hline
\end{tabular}

NOTE: We do not show in this table all the control variables included in the regressions. The reference categories for the control variables are wave 1, Motul, female, do not speak Maya, do not read write a message in Spanish, no schooling, single, and the missing category for respondent income and wealth. SOURCE: Baseline and first follow-up ENCAHEY, 2008 and 2009.

$* *=$ significant at $5 \%$ level of confidence. $*=$ significant at $10 \%$ level of confidence

Table 5h: Interaction Effects

\begin{tabular}{|c|c|c|c|c|c|c|c|c|}
\hline & Total food & $\begin{array}{l}\text { Received free } \\
\text { food (yes-no } \\
{[1-0] \text { ) }}\end{array}$ & $\begin{array}{l}\text { Eat diary } \\
\text { products: } \\
\text { milk, cheese, } \\
\text { yogurt (at } \\
\text { least once a } \\
\text { day, at least } \\
\text { once a w }\end{array}$ & $\begin{array}{l}\text { Eat eggs, } \\
\text { beans or lentil } \\
\text { (at least once } \\
\text { a day, at least } \\
\text { once a week, } \\
\text { several ti }\end{array}$ & $\begin{array}{l}\text { Eat meat, } \\
\text { poultry or } \\
\text { fish (at least } \\
\text { once a day, at } \\
\text { least once a } \\
\text { week, several } \\
\text { ti }\end{array}$ & $\begin{array}{l}\text { Eat fruit or } \\
\text { vegetables (at } \\
\text { least once a } \\
\text { day, at least } \\
\text { once a week, } \\
\text { several time }\end{array}$ & $\begin{array}{l}\text { Eat tortillas, } \\
\text { bread, } \\
\text { crackers or } \\
\text { other cereals } \\
\text { (at least once } \\
\text { a day, at least } \\
\text { o }\end{array}$ & $\begin{array}{l}\text { Worked for } \\
\text { pay last } \\
\text { month (yes- } \\
\text { no [1-0]) }\end{array}$ \\
\hline \multirow[t]{2}{*}{ Wave } & 44.494 & 0.088 & -0.165 & 0.175 & 0.092 & -0.057 & -0.114 & -0.004 \\
\hline & (26.681) & $(0.024)^{* *}$ & (0.089) & $(0.070)^{*}$ & $(0.071)$ & $(0.076)$ & $(0.033)^{* *}$ & $(0.020)$ \\
\hline \multirow[t]{2}{*}{ Valladolid } & -38.485 & -0.051 & 0.190 & 0.143 & 0.027 & 0.012 & -0.028 & -0.038 \\
\hline & (23.917) & $(0.017)^{* *}$ & $(0.067)^{* *}$ & $(0.050)^{* *}$ & $(0.051)$ & (0.058) & $(0.021)$ & $(0.015)^{*}$ \\
\hline \multirow{3}{*}{$\begin{array}{l}\text { Valladolid } \\
\text { wave } 2\end{array}$} & -5.287 & -0.017 & 0.020 & -0.067 & -0.177 & -0.005 & 0.042 & 0.030 \\
\hline & & & & & & & & \\
\hline & (46.795) & (0.031) & (0.099) & (0.079) & $(0.078)^{*}$ & (0.089) & (0.038) & (0.021) \\
\hline \multirow{2}{*}{$\begin{array}{l}\text { Effect for } \\
\text { income } \\
\text { quartile } 1\end{array}$} & -17.360 & -0.036 & -0.115 & 0.070 & 0.203 & -0.048 & -0.066 & -0.093 \\
\hline & (45.605) & $(0.042)$ & $(0.131)$ & $(0.097)$ & $(0.098)^{*}$ & (0.109) & $(0.042)$ & $(0.027)^{* *}$ \\
\hline \multirow{2}{*}{$\begin{array}{l}\text { Effect for } \\
\text { income } \\
\text { quartile } 2\end{array}$} & -25.334 & -0.049 & 0.163 & 0.105 & 0.209 & 0.011 & -0.067 & -0.099 \\
\hline & (45.764) & $(0.043)$ & $(0.126)$ & $(0.092)$ & $(0.101)^{*}$ & $(0.112)$ & $(0.046)$ & $(0.028) * *$ \\
\hline Effect for & -31.010 & -0.035 & -0.038 & 0.087 & 0.191 & 0.179 & -0.007 & 0.011 \\
\hline
\end{tabular}




\begin{tabular}{|c|c|c|c|c|c|c|c|c|}
\hline \multicolumn{9}{|l|}{ income } \\
\hline & $(45.926)$ & $(0.045)$ & $(0.149)$ & $(0.108)$ & $(0.110)$ & $(0.121)$ & $(0.052)$ & $(0.035)$ \\
\hline \multirow{2}{*}{$\begin{array}{l}\text { Effect for } \\
\text { income } \\
\text { quartile } 4\end{array}$} & 34.350 & -0.068 & -0.212 & 0.180 & 0.340 & 0.183 & -0.029 & -0.066 \\
\hline & (59.201) & $(0.036)$ & (0.133) & $(0.100)$ & $(0.102)^{* *}$ & $(0.117)$ & $(0.050)$ & $(0.035)$ \\
\hline \multirow{2}{*}{$\begin{array}{l}\text { Effect on } \\
\text { males }\end{array}$} & 41.736 & -0.011 & -0.003 & -0.026 & 0.011 & -0.107 & -0.024 & -0.070 \\
\hline & (21.856) & $(0.023)$ & $(0.085)$ & $(0.064)$ & $(0.065)$ & $(0.072)$ & $(0.032)$ & $(0.021) * *$ \\
\hline Constant & $\begin{array}{l}-246.189 \\
(1,374.469)\end{array}$ & $\begin{array}{l}-1.327 \\
(0.777)\end{array}$ & $\begin{array}{l}4.136 \\
(3.532)\end{array}$ & $\begin{array}{l}1.434 \\
(1.985)\end{array}$ & $\begin{array}{l}2.958 \\
(2.136)\end{array}$ & $\begin{array}{l}5.113 \\
(2.716)\end{array}$ & $\begin{array}{l}0.687 \\
(0.665)\end{array}$ & $\begin{array}{l}3.372 \\
(0.627) * *\end{array}$ \\
\hline Observations & 3028 & 3621 & 4002 & 4000 & 4002 & 4002 & 4003 & 4006 \\
\hline R-squared & 0.11 & 0.07 & 0.09 & 0.02 & 0.04 & 0.05 & 0.01 & 0.18 \\
\hline $\begin{array}{l}\text { F-test SES } \\
\text { interactions }\end{array}$ & 0.22 & 0.28 & 2.26 & 0.36 & 0.65 & 1.55 & 0.63 & 2.74 \\
\hline Prob $>F$ & 0.881 & 0.838 & 0.079 & 0.785 & 0.580 & 0.200 & 0.597 & 0.042 \\
\hline
\end{tabular}

NOTE: We do not show in this table all the control variables included in the regressions. The reference categories for the control variables are wave 1, Motul, female, do not speak Maya, do not read write a message in Spanish, no schooling, single, and the missing category for respondent income and wealth. SOURCE: Baseline and first follow-up ENCAHEY, 2008 and 2009.

$* *=$ significant at $5 \%$ level of confidence. $*=$ significant at $10 \%$ level of confidence 
Table 5i: Interaction Effects

\begin{tabular}{|c|c|c|c|c|c|c|}
\hline & $\begin{array}{l}\text { Immediate recall } \\
\text { (number of words) }\end{array}$ & $\begin{array}{l}\text { Delayed recall } \\
\text { (number of words) }\end{array}$ & $\begin{array}{l}\text { Feel fear someone } \\
\text { robbing you } \\
\text { (never, } \\
\text { sometimes, } \\
\text { usually, always } \\
{[1-4] \text { ) }}\end{array}$ & $\begin{array}{l}\text { Feel fear someone } \\
\text { close to you will } \\
\text { take your money } \\
\text { (never, } \\
\text { sometimes, } \\
\text { usually, }\end{array}$ & $\begin{array}{l}\text { Money in a safe } \\
\text { place (yes-no [1- } \\
0] \text { ) }\end{array}$ & $\begin{array}{l}\text { Feel verbally or } \\
\text { physically abused } \\
\text { (never, } \\
\text { sometimes, } \\
\text { usually, always } \\
\text { [1-4]) }\end{array}$ \\
\hline \multirow[t]{2}{*}{ Wave } & -0.024 & 0.104 & -0.191 & -0.028 & -0.006 & -0.019 \\
\hline & $(0.101)$ & $(0.128)$ & $(0.061)^{* *}$ & $(0.045)$ & $(0.014)$ & $(0.029)$ \\
\hline \multirow[t]{2}{*}{ Valladolid } & 0.099 & 0.188 & -0.168 & -0.081 & -0.018 & -0.029 \\
\hline & $(0.076)$ & $(0.090) *$ & $(0.043)^{* *}$ & $(0.029) * *$ & $(0.008) *$ & $(0.020)$ \\
\hline \multirow[t]{2}{*}{ Valladolid wave 2} & 0.106 & 0.488 & 0.033 & -0.026 & -0.011 & -0.033 \\
\hline & $(0.118)$ & $(0.146)^{* *}$ & $(0.069)$ & $(0.050)$ & $(0.014)$ & $(0.039)$ \\
\hline \multirow{2}{*}{$\begin{array}{l}\text { Effect for income } \\
\text { quartile } 1\end{array}$} & 0.058 & -0.156 & -0.045 & 0.076 & 0.008 & -0.021 \\
\hline & $(0.157)$ & $(0.187)$ & $(0.094)$ & (0.059) & $(0.016)$ & $(0.046)$ \\
\hline \multirow{2}{*}{$\begin{array}{l}\text { Effect for income } \\
\text { quartile } 2\end{array}$} & 0.203 & -0.077 & 0.048 & 0.026 & 0.003 & -0.012 \\
\hline & $(0.165)$ & $(0.196)$ & $(0.092)$ & $(0.066)$ & $(0.016)$ & $(0.049)$ \\
\hline \multirow{2}{*}{$\begin{array}{l}\text { Effect for income } \\
\text { quartile } 3\end{array}$} & 0.193 & 0.211 & -0.116 & -0.039 & -0.001 & 0.067 \\
\hline & $(0.175)$ & $(0.212)$ & $(0.092)$ & $(0.066)$ & $(0.019)$ & $(0.059)$ \\
\hline \multirow{2}{*}{$\begin{array}{l}\text { Effect for income } \\
\text { quartile } 4\end{array}$} & 0.323 & 0.069 & -0.055 & 0.072 & 0.032 & -0.019 \\
\hline & $(0.180)$ & $(0.212)$ & $(0.089)$ & $(0.061)$ & $(0.018)$ & $(0.044)$ \\
\hline \multirow[t]{2}{*}{ Effect on males } & 0.048 & 0.136 & -0.033 & 0.003 & -0.004 & 0.032 \\
\hline & $(0.109)$ & $(0.129)$ & $(0.060)$ & $(0.041)$ & $(0.011)$ & $(0.032)$ \\
\hline Constant & $\begin{array}{l}8.086 \\
(3.699) *\end{array}$ & $\begin{array}{l}13.891 \\
(4.061)^{* *}\end{array}$ & $\begin{array}{l}2.957 \\
(2.399)\end{array}$ & $\begin{array}{l}1.658 \\
(1.740)\end{array}$ & $\begin{array}{l}-0.357 \\
(0.335)\end{array}$ & $\begin{array}{l}1.187 \\
(0.950)\end{array}$ \\
\hline Observations & 4011 & 4011 & 3253 & 3251 & 3250 & 3253 \\
\hline R-squared & 0.17 & 0.15 & 0.03 & 0.02 & 0.01 & 0.01 \\
\hline $\begin{array}{l}\text { F-test SES } \\
\text { interactions }\end{array}$ & 0.59 & 0.93 & 0.83 & 0.99 & 0.79 & 0.80 \\
\hline Prob $>F$ & 0.620 & 0.424 & 0.480 & 0.398 & 0.500 & 0.492 \\
\hline
\end{tabular}


NOTE: We do not show in this table all the control variables included in the regressions. The reference categories for the control variables are wave 1, Motul, female, do not speak Maya, do not read write a message in Spanish, no schooling, single, and the missing category for respondent income and wealth. SOURCE: Baseline and first follow-up ENCAHEY, 2008 and 2009.

$* *=$ significant at $5 \%$ level of confidence. $*=$ significant at $10 \%$ level of confidence 
Table 6a: Treatment regressions

\begin{tabular}{|c|c|c|c|c|c|c|c|}
\hline & $\begin{array}{l}\text { Self-reported } \\
\text { health } \\
\text { (excellent, very } \\
\text { good, good, } \\
\text { fair, poor [1-5]) }\end{array}$ & $\begin{array}{l}\text { Subjective } \\
\text { mortality } \\
\text { expectation } \\
\text { (chances to } \\
\text { leave at least } 10 \\
\text { years more [1- } \\
100\end{array}$ & $\begin{array}{l}\text { Feel sad, } \\
\text { blue or } \\
\text { depressed for } \\
2 \text { weeks or } \\
\text { more during } \\
\text { the last } 3 \\
\text { months (yes- } \\
\text { no }\end{array}$ & $\begin{array}{l}\text { Satisfied } \\
\text { relation fam. } \\
\text { members (very } \\
\text { satisfied-very } \\
\text { unsatisfied [1- } \\
\text { 5]) }\end{array}$ & $\begin{array}{l}\text { Satisfied hh } \\
\text { income (very } \\
\text { satisfied-very } \\
\text { unsatisfied [1- } \\
\text { 5]) }\end{array}$ & $\begin{array}{l}\text { Satisfied social } \\
\text { contacts (very } \\
\text { satisfied-very } \\
\text { unsatisfied [1- } \\
\text { 5]) }\end{array}$ & $\begin{array}{l}\text { Satisfied with } \\
\text { job (very } \\
\text { satisfied-very } \\
\text { unsatisfied [1- } \\
\text { 5]) }\end{array}$ \\
\hline Wave & $\begin{array}{l}-0.410 \\
(0.075)^{* *}\end{array}$ & $\begin{array}{l}0.401 \\
(2.401)\end{array}$ & $\begin{array}{l}-0.530 \\
(0.098) * *\end{array}$ & $\begin{array}{l}-0.188 \\
(0.086)^{*}\end{array}$ & $\begin{array}{l}-0.256 \\
(0.083)^{* *}\end{array}$ & $\begin{array}{l}-0.139 \\
(0.093)\end{array}$ & $\begin{array}{l}-0.261 \\
(0.083)^{* *}\end{array}$ \\
\hline Valladolid & $\begin{array}{l}0.144 \\
(0.054)^{* *}\end{array}$ & $\begin{array}{l}-4.302 \\
(1.575)^{* *}\end{array}$ & $\begin{array}{l}-0.199 \\
(0.066)^{* *}\end{array}$ & $\begin{array}{l}0.009 \\
(0.062)\end{array}$ & $\begin{array}{l}0.013 \\
(0.061)\end{array}$ & $\begin{array}{l}-0.013 \\
(0.064)\end{array}$ & $\begin{array}{l}-0.025 \\
(0.061)\end{array}$ \\
\hline Valladolid wave 2 & $\begin{array}{l}0.146 \\
(0.062)^{*}\end{array}$ & $\begin{array}{l}-3.107 \\
(2.219)\end{array}$ & $\begin{array}{l}-0.045 \\
(0.088)\end{array}$ & $\begin{array}{l}0.095 \\
(0.077)\end{array}$ & $\begin{array}{l}-0.065 \\
(0.076)\end{array}$ & $\begin{array}{l}0.085 \\
(0.081)\end{array}$ & $\begin{array}{l}0.073 \\
(0.073)\end{array}$ \\
\hline Age & $\begin{array}{l}0.079 \\
(0.060)\end{array}$ & $\begin{array}{l}-5.887 \\
(1.973)^{* *}\end{array}$ & $\begin{array}{l}0.098 \\
(0.081)\end{array}$ & $\begin{array}{l}-0.021 \\
(0.080)\end{array}$ & $\begin{array}{l}-0.103 \\
(0.064)\end{array}$ & $\begin{array}{l}0.034 \\
(0.075)\end{array}$ & $\begin{array}{l}0.004 \\
(0.077)\end{array}$ \\
\hline Age squared & $\begin{array}{l}-0.000 \\
(0.000)\end{array}$ & $\begin{array}{l}0.036 \\
(0.012)^{* *}\end{array}$ & $\begin{array}{l}-0.001 \\
(0.001)\end{array}$ & $\begin{array}{l}0.000 \\
(0.000)\end{array}$ & $\begin{array}{l}0.001 \\
(0.000)\end{array}$ & $\begin{array}{l}-0.000 \\
(0.000)\end{array}$ & $\begin{array}{l}0.000 \\
(0.000)\end{array}$ \\
\hline Gender (male=1) & $\begin{array}{l}-0.137 \\
(0.041)^{* *}\end{array}$ & $\begin{array}{l}3.756 \\
(1.111)^{* *}\end{array}$ & $\begin{array}{l}-0.350 \\
(0.049)^{* *}\end{array}$ & $\begin{array}{l}0.037 \\
(0.047)\end{array}$ & $\begin{array}{l}0.047 \\
(0.042)\end{array}$ & $\begin{array}{l}-0.077 \\
(0.046)\end{array}$ & $\begin{array}{l}0.037 \\
(0.047)\end{array}$ \\
\hline Speaks Maya & $\begin{array}{l}-0.072 \\
(0.065)\end{array}$ & $\begin{array}{l}0.850 \\
(1.746)\end{array}$ & $\begin{array}{l}-0.106 \\
(0.075)\end{array}$ & $\begin{array}{l}0.081 \\
(0.071)\end{array}$ & $\begin{array}{l}0.035 \\
(0.069)\end{array}$ & $\begin{array}{l}0.116 \\
(0.070)\end{array}$ & $\begin{array}{l}0.036 \\
(0.074)\end{array}$ \\
\hline Reads/writes Spanish & $\begin{array}{l}0.004 \\
(0.056)\end{array}$ & $\begin{array}{l}-1.714 \\
(1.759)\end{array}$ & $\begin{array}{l}-0.131 \\
(0.072)\end{array}$ & $\begin{array}{l}-0.124 \\
(0.064)\end{array}$ & $\begin{array}{l}-0.006 \\
(0.065)\end{array}$ & $\begin{array}{l}-0.123 \\
(0.068)\end{array}$ & $\begin{array}{l}-0.107 \\
(0.063)\end{array}$ \\
\hline Lives alone & $\begin{array}{l}-0.071 \\
(0.073)\end{array}$ & $\begin{array}{l}1.122 \\
(2.072)\end{array}$ & $\begin{array}{l}0.079 \\
(0.088)\end{array}$ & $\begin{array}{l}0.164 \\
(0.088)\end{array}$ & $\begin{array}{l}0.137 \\
(0.080)\end{array}$ & $\begin{array}{l}0.085 \\
(0.085)\end{array}$ & $\begin{array}{l}0.065 \\
(0.086)\end{array}$ \\
\hline household size & $\begin{array}{l}-0.006 \\
(0.010)\end{array}$ & $\begin{array}{l}-0.265 \\
(0.279)\end{array}$ & $\begin{array}{l}0.001 \\
(0.012)\end{array}$ & $\begin{array}{l}0.004 \\
(0.012)\end{array}$ & $\begin{array}{l}0.013 \\
(0.012)\end{array}$ & $\begin{array}{l}0.031 \\
(0.012)^{*}\end{array}$ & $\begin{array}{l}0.019 \\
(0.012)\end{array}$ \\
\hline Incomplete primary & $\begin{array}{l}0.007 \\
(0.045)\end{array}$ & $\begin{array}{l}1.432 \\
(1.339)\end{array}$ & $\begin{array}{l}0.047 \\
(0.057)\end{array}$ & $\begin{array}{l}-0.108 \\
(0.050)^{*}\end{array}$ & $\begin{array}{l}-0.035 \\
(0.052)\end{array}$ & $\begin{array}{l}0.003 \\
(0.054)\end{array}$ & $\begin{array}{l}-0.109 \\
(0.052)^{*}\end{array}$ \\
\hline Primary & $\begin{array}{l}-0.233 \\
(0.073)^{* *}\end{array}$ & $\begin{array}{l}0.987 \\
(1.926)\end{array}$ & $\begin{array}{l}-0.164 \\
(0.088)\end{array}$ & $\begin{array}{l}-0.671 \\
(0.091)^{* *}\end{array}$ & $\begin{array}{l}-0.156 \\
(0.079)^{*}\end{array}$ & $\begin{array}{l}-0.472 \\
(0.089)^{* *}\end{array}$ & $\begin{array}{l}-0.396 \\
(0.086)^{* *}\end{array}$ \\
\hline Couple & $\begin{array}{l}0.160 \\
(0.109)\end{array}$ & $\begin{array}{l}4.545 \\
(2.644)\end{array}$ & $\begin{array}{l}0.220 \\
(0.124)\end{array}$ & $\begin{array}{l}-0.076 \\
(0.101)\end{array}$ & $\begin{array}{l}0.109 \\
(0.096) \\
\end{array}$ & $\begin{array}{l}-0.008 \\
(0.090)\end{array}$ & $\begin{array}{l}0.008 \\
(0.092) \\
\end{array}$ \\
\hline
\end{tabular}




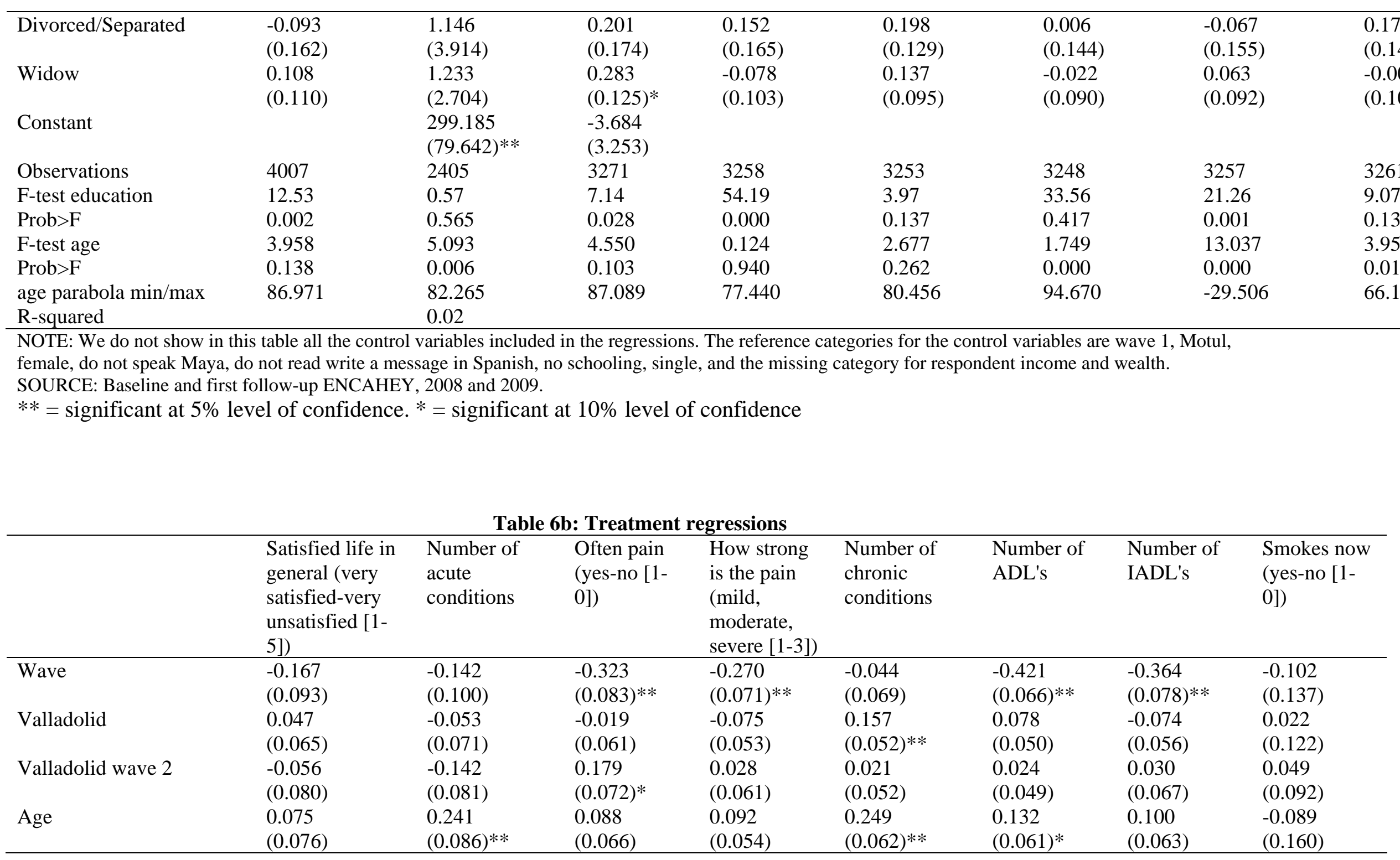




\begin{tabular}{|c|c|c|c|c|c|c|c|c|}
\hline Age squared & $\begin{array}{l}-0.000 \\
(0.000)\end{array}$ & $\begin{array}{l}-0.001 \\
(0.001)^{* *}\end{array}$ & $\begin{array}{l}-0.001 \\
(0.000)\end{array}$ & $\begin{array}{l}-0.001 \\
(0.000)\end{array}$ & $\begin{array}{l}-0.002 \\
(0.000)^{* *}\end{array}$ & $\begin{array}{l}-0.001 \\
(0.000)\end{array}$ & $\begin{array}{l}-0.000 \\
(0.000)\end{array}$ & $\begin{array}{l}0.000 \\
(0.001)\end{array}$ \\
\hline Gender (male=1) & $\begin{array}{l}-0.061 \\
(0.046)\end{array}$ & $\begin{array}{l}-0.020 \\
(0.059)\end{array}$ & $\begin{array}{l}-0.100 \\
(0.048) *\end{array}$ & $\begin{array}{l}-0.110 \\
(0.042)^{* *}\end{array}$ & $\begin{array}{l}-0.296 \\
(0.044)^{* *}\end{array}$ & $\begin{array}{l}-0.251 \\
(0.042)^{* *}\end{array}$ & $\begin{array}{l}-0.242 \\
(0.044)^{* *}\end{array}$ & $\begin{array}{l}0.958 \\
(0.143)^{* *}\end{array}$ \\
\hline Speaks Maya & $\begin{array}{l}0.120 \\
(0.071)\end{array}$ & $\begin{array}{l}-0.073 \\
(0.079)\end{array}$ & $\begin{array}{l}-0.025 \\
(0.069)\end{array}$ & $\begin{array}{l}-0.022 \\
(0.058)\end{array}$ & $\begin{array}{l}0.043 \\
(0.061)\end{array}$ & $\begin{array}{l}-0.159 \\
(0.060)^{* *}\end{array}$ & $\begin{array}{l}-0.142 \\
(0.064)^{*}\end{array}$ & $\begin{array}{l}-0.145 \\
(0.140)\end{array}$ \\
\hline Reads/writes Spanish & $\begin{array}{l}-0.088 \\
(0.072)\end{array}$ & $\begin{array}{l}0.002 \\
(0.080)\end{array}$ & $\begin{array}{l}-0.035 \\
(0.064)\end{array}$ & $\begin{array}{l}-0.045 \\
(0.054)\end{array}$ & $\begin{array}{l}-0.051 \\
(0.053)\end{array}$ & $\begin{array}{l}-0.191 \\
(0.051)^{* *}\end{array}$ & $\begin{array}{l}-0.094 \\
(0.059)\end{array}$ & $\begin{array}{l}0.086 \\
(0.128)\end{array}$ \\
\hline Lives alone & $\begin{array}{l}0.103 \\
(0.084)\end{array}$ & $\begin{array}{l}-0.118 \\
(0.106)\end{array}$ & $\begin{array}{l}0.001 \\
(0.082)\end{array}$ & $\begin{array}{l}-0.059 \\
(0.071)\end{array}$ & $\begin{array}{l}-0.062 \\
(0.073)\end{array}$ & $\begin{array}{l}-0.025 \\
(0.071)\end{array}$ & $\begin{array}{l}-0.023 \\
(0.076)\end{array}$ & $\begin{array}{l}0.055 \\
(0.183)\end{array}$ \\
\hline household size & $\begin{array}{l}0.002 \\
(0.012)\end{array}$ & $\begin{array}{l}0.020 \\
(0.014)\end{array}$ & $\begin{array}{l}-0.001 \\
(0.012)\end{array}$ & $\begin{array}{l}-0.003 \\
(0.010)\end{array}$ & $\begin{array}{l}-0.008 \\
(0.010)\end{array}$ & $\begin{array}{l}-0.006 \\
(0.010)\end{array}$ & $\begin{array}{l}-0.004 \\
(0.011)\end{array}$ & $\begin{array}{l}-0.031 \\
(0.027)\end{array}$ \\
\hline Incomplete primary & $\begin{array}{l}0.002 \\
(0.053)\end{array}$ & $\begin{array}{l}0.096 \\
(0.070)\end{array}$ & $\begin{array}{l}-0.076 \\
(0.052)\end{array}$ & $\begin{array}{l}-0.084 \\
(0.044)\end{array}$ & $\begin{array}{l}0.068 \\
(0.047)\end{array}$ & $\begin{array}{l}0.092 \\
(0.043)^{*}\end{array}$ & $\begin{array}{l}-0.028 \\
(0.048)\end{array}$ & $\begin{array}{l}0.066 \\
(0.117)\end{array}$ \\
\hline Primary & $\begin{array}{l}-0.368 \\
(0.086)^{* *}\end{array}$ & $\begin{array}{l}0.387 \\
(0.095)^{* *}\end{array}$ & $\begin{array}{l}-0.394 \\
(0.081)^{* *}\end{array}$ & $\begin{array}{l}-0.284 \\
(0.075)^{* *}\end{array}$ & $\begin{array}{l}0.019 \\
(0.075)\end{array}$ & $\begin{array}{l}-0.105 \\
(0.069)\end{array}$ & $\begin{array}{l}-0.195 \\
(0.077)^{*}\end{array}$ & $\begin{array}{l}0.266 \\
(0.167)\end{array}$ \\
\hline Couple & $\begin{array}{l}0.094 \\
(0.103)\end{array}$ & $\begin{array}{l}0.263 \\
(0.142)\end{array}$ & $\begin{array}{l}0.288 \\
(0.110)^{* *}\end{array}$ & $\begin{array}{l}0.280 \\
(0.095)^{* *}\end{array}$ & $\begin{array}{l}0.179 \\
(0.097)\end{array}$ & $\begin{array}{l}0.072 \\
(0.099)\end{array}$ & $\begin{array}{l}-0.039 \\
(0.102)\end{array}$ & $\begin{array}{l}0.286 \\
(0.288)\end{array}$ \\
\hline Divorced/Separated & $\begin{array}{l}0.104 \\
(0.150)\end{array}$ & $\begin{array}{l}0.584 \\
(0.198)^{* *}\end{array}$ & $\begin{array}{l}0.350 \\
(0.159) *\end{array}$ & $\begin{array}{l}0.365 \\
(0.138)^{* *}\end{array}$ & $\begin{array}{l}0.317 \\
(0.145)^{*}\end{array}$ & $\begin{array}{l}0.100 \\
(0.136)\end{array}$ & $\begin{array}{l}-0.210 \\
(0.151)\end{array}$ & $\begin{array}{l}-0.271 \\
(0.487)\end{array}$ \\
\hline Widow & $\begin{array}{l}0.064 \\
(0.103)\end{array}$ & $\begin{array}{l}0.347 \\
(0.142)^{*}\end{array}$ & $\begin{array}{l}0.163 \\
(0.108)\end{array}$ & $\begin{array}{l}0.193 \\
(0.093)^{*}\end{array}$ & $\begin{array}{l}0.114 \\
(0.097)\end{array}$ & $\begin{array}{l}0.131 \\
(0.098)\end{array}$ & $\begin{array}{l}-0.011 \\
(0.102)\end{array}$ & $\begin{array}{l}0.240 \\
(0.280)\end{array}$ \\
\hline Constant & & & $\begin{array}{l}-3.366 \\
(2.691)\end{array}$ & & & & & $\begin{array}{l}1.253 \\
(6.537)\end{array}$ \\
\hline Observations & 3257 & 4011 & 4006 & 4003 & 4011 & 3769 & 4002 & 4008 \\
\hline F-test education & 22.43 & 17.00 & 24.39 & 14.41 & 2.29 & 11.46 & 6.66 & 2.60 \\
\hline Prob $>F$ & 0.000 & 0.000 & 0.169 & 0.001 & 0.319 & 0.000 & 0.036 & 0.272 \\
\hline F-test age & 1.210 & 8.035 & 3.552 & 4.038 & 28.980 & 70.328 & 79.440 & 1.849 \\
\hline Prob $>F$ & 0.546 & 0.018 & 0.000 & 0.133 & 0.000 & 0.003 & 0.000 & 0.397 \\
\hline age parabola min/max & 83.125 & 81.065 & 86.866 & 85.392 & 78.344 & 102.754 & 116.677 & 93.282 \\
\hline
\end{tabular}

NOTE: We do not show in this table all the control variables included in the regressions. The reference categories for the control variables are wave 1, Motul, female, do not speak Maya, do not read write a message in Spanish, no schooling, single, and the missing category for respondent income and wealth. SOURCE: Baseline and first follow-up ENCAHEY, 2008 and 2009.

** = significant at $5 \%$ level of confidence. ${ }^{*}=$ significant at $10 \%$ level of confidence 
Table 6c: Treatment regressions

\begin{tabular}{|c|c|c|c|c|c|c|c|c|}
\hline & $\begin{array}{l}\text { Number of } \\
\text { cigarettes in a } \\
\text { day }\end{array}$ & $\begin{array}{l}\text { Drink } \\
\text { alcoholic } \\
\text { beverages } \\
\text { (yes-no [1- } \\
0] \text { ) }\end{array}$ & $\begin{array}{l}\text { Number of } \\
\text { days a week } \\
\text { drinks } \\
\text { alcoholic } \\
\text { beverages }\end{array}$ & $\begin{array}{l}\text { Number of } \\
\text { drinks per } \\
\text { day }\end{array}$ & $\begin{array}{l}\text { Visited a } \\
\text { doctor (yes- } \\
\text { no [1-0]) }\end{array}$ & $\begin{array}{l}\text { Number of } \\
\text { doctor visits }\end{array}$ & $\begin{array}{l}\text { Visited a } \\
\text { folk healer } \\
\text { (yes-no [1- } \\
0] \text { ) }\end{array}$ & $\begin{array}{l}\text { Number of } \\
\text { folk healer } \\
\text { visits }\end{array}$ \\
\hline \multirow[t]{2}{*}{ Wave } & -1.949 & 0.376 & 0.136 & 0.947 & 0.226 & 0.175 & -0.183 & -0.148 \\
\hline & (1.793) & $(0.091)^{* *}$ & (0.118) & $(0.622)$ & $(0.088) *$ & $(0.082)^{*}$ & $(0.167)$ & $(0.160)$ \\
\hline \multirow[t]{2}{*}{ Valladolid } & 0.905 & 0.120 & -0.176 & -1.178 & 0.185 & 0.161 & -0.321 & -0.320 \\
\hline & (1.097) & $(0.065)$ & $(0.080)^{*}$ & $(0.400)^{* *}$ & $(0.061)^{* *}$ & $(0.056)^{* *}$ & $(0.115)^{* *}$ & $(0.116)^{* *}$ \\
\hline \multirow[t]{2}{*}{ Valladolid wave 2} & 1.153 & -0.521 & -0.300 & -1.740 & 0.212 & 0.177 & -0.170 & -0.180 \\
\hline & (1.577) & $(0.077)^{* *}$ & $(0.092)^{* *}$ & $(0.561)^{* *}$ & $(0.074)^{* *}$ & $(0.065)^{* *}$ & $(0.154)$ & $(0.152)$ \\
\hline \multirow[t]{2}{*}{ Age } & -0.313 & -0.022 & 0.027 & 0.149 & 0.036 & 0.058 & 0.134 & 0.163 \\
\hline & (1.374) & $(0.071)$ & (0.110) & $(0.498)$ & $(0.078)$ & $(0.067)$ & (0.118) & $(0.117)$ \\
\hline \multirow[t]{2}{*}{ Age squared } & 0.001 & 0.000 & -0.000 & -0.002 & -0.000 & -0.000 & -0.001 & -0.001 \\
\hline & (0.009) & $(0.000)$ & $(0.001)$ & $(0.003)$ & $(0.000)$ & $(0.000)$ & $(0.001)$ & $(0.001)$ \\
\hline \multirow[t]{2}{*}{ Gender (male=1) } & 8.731 & 1.023 & 0.353 & 1.940 & -0.199 & -0.188 & 0.262 & 0.248 \\
\hline & $(1.288)^{* *}$ & $(0.049)^{* *}$ & $(0.060)^{* *}$ & $(0.303)^{* *}$ & $(0.046) * *$ & $(0.042)^{* *}$ & $(0.080) * *$ & $(0.080)^{* *}$ \\
\hline \multirow[t]{2}{*}{ Speaks Maya } & -1.749 & -0.242 & -0.107 & -0.612 & 0.130 & 0.144 & -0.188 & -0.145 \\
\hline & (1.266) & $(0.073)^{* *}$ & $(0.088)$ & $(0.449)$ & $(0.071)$ & $(0.068)^{*}$ & $(0.123)$ & $(0.117)$ \\
\hline \multirow[t]{2}{*}{ Reads/writes Spanish } & 0.721 & 0.101 & 0.178 & 0.897 & 0.093 & 0.075 & -0.283 & -0.276 \\
\hline & (1.356) & $(0.067)$ & $(0.085)^{*}$ & $(0.472)$ & $(0.063)$ & $(0.058)$ & $(0.123)^{*}$ & $(0.121)^{*}$ \\
\hline \multirow[t]{2}{*}{ Lives alone } & 1.129 & 0.061 & -0.030 & 0.056 & -0.103 & -0.103 & 0.047 & 0.045 \\
\hline & $(1.412)$ & $(0.091)$ & $(0.105)$ & $(0.497)$ & $(0.081)$ & $(0.075)$ & $(0.144)$ & $(0.143)$ \\
\hline \multirow[t]{2}{*}{ household size } & -0.245 & -0.001 & -0.031 & -0.103 & -0.007 & -0.003 & -0.026 & -0.025 \\
\hline & $(0.216)$ & $(0.012)$ & $(0.015)^{*}$ & $(0.074)$ & $(0.011)$ & $(0.011)$ & $(0.022)$ & $(0.022)$ \\
\hline \multirow[t]{2}{*}{ Incomplete primary } & 0.734 & 0.221 & 0.299 & 1.563 & 0.131 & 0.084 & -0.097 & -0.098 \\
\hline & $(1.028)$ & $(0.056)^{* *}$ & $(0.072)^{* *}$ & $(0.363)^{* *}$ & $(0.051)^{*}$ & $(0.045)$ & $(0.089)$ & $(0.087)$ \\
\hline \multirow[t]{2}{*}{ Primary } & 3.384 & 0.282 & 0.500 & 2.244 & 0.223 & 0.213 & -0.502 & -0.499 \\
\hline & $(1.313)^{* *}$ & $(0.095)^{* *}$ & $(0.103)^{* *}$ & $(0.489) * *$ & $(0.081)^{* *}$ & $(0.072)^{* *}$ & $(0.169)^{* *}$ & $(0.165)^{* *}$ \\
\hline \multirow[t]{2}{*}{ Couple } & 4.120 & 0.096 & 0.173 & 0.709 & 0.208 & 0.203 & 0.142 & 0.064 \\
\hline & $(2.407)$ & $(0.120)$ & $(0.143)$ & $(0.668)$ & $(0.105)^{*}$ & $(0.100)^{*}$ & $(0.194)$ & (0.199) \\
\hline Divorced/Separated & -1.020 & 0.294 & 0.156 & 0.532 & -0.002 & 0.094 & -0.237 & -0.302 \\
\hline
\end{tabular}




\begin{tabular}{|c|c|c|c|c|c|c|c|c|}
\hline & $(4.102)$ & $(0.179)$ & $(0.196)$ & $(0.987)$ & $(0.155)$ & $(0.152)$ & $(0.342)$ & $(0.341)$ \\
\hline \multirow[t]{2}{*}{ Widow } & 3.834 & 0.099 & 0.156 & 0.495 & 0.270 & 0.245 & 0.160 & 0.079 \\
\hline & $(2.435)$ & $(0.118)$ & $(0.142)$ & $(0.673)$ & $(0.105)^{*}$ & $(0.099) *$ & (0.189) & $(0.193)$ \\
\hline \multirow[t]{2}{*}{ Constant } & -11.433 & 0.861 & & -8.075 & -1.719 & & -6.630 & \\
\hline & (55.267) & (2.895) & & (20.001) & (3.149) & & $(4.802)$ & \\
\hline Observations & 4011 & 4010 & 3983 & 3992 & 4008 & 4004 & 4009 & 4009 \\
\hline F-test education & 3.84 & 17.72 & 27.21 & 12.54 & 9.98 & 9.10 & 8.90 & 9.20 \\
\hline Prob $>F$ & 0.022 & 0.000 & 0.000 & 0.000 & 0.007 & 0.011 & 0.012 & 0.376 \\
\hline F-test age & 1.240 & 16.148 & 20.111 & 15.241 & 11.411 & 20.246 & 1.314 & 1.954 \\
\hline Prob $>F$ & 0.289 & 0.000 & 0.000 & 0.000 & 0.003 & 0.000 & 0.519 & 0.010 \\
\hline age parabola min/max & 122.058 & 310.743 & 41.954 & 42.270 & 60.073 & 64.665 & 81.912 & 81.949 \\
\hline
\end{tabular}

NOTE: We do not show in this table all the control variables included in the regressions. The reference categories for the control variables are wave 1, Motul, female, do not speak Maya, do not read write a message in Spanish, no schooling, single, and the missing category for respondent income and wealth. SOURCE: Baseline and first follow-up ENCAHEY, 2008 and 2009.

$* *=$ significant at 5\% level of confidence. * = significant at $10 \%$ level of confidence

Table 6d: Treatment regressions

\begin{tabular}{|c|c|c|c|c|c|c|c|}
\hline & $\begin{array}{l}\text { Visited a } \\
\text { dentist (yes- } \\
\text { no [1-0]) }\end{array}$ & $\begin{array}{l}\text { Number of } \\
\text { dentist visits }\end{array}$ & $\begin{array}{l}\text { Outpatient } \\
\text { procedures } \\
\text { (yes-no [1-0]) }\end{array}$ & $\begin{array}{l}\text { Consulted a } \\
\text { pharmacist } \\
\text { (yes-no [1-0]) }\end{array}$ & $\begin{array}{l}\text { Bought no } \\
\text { medicines } \\
\text { because they } \\
\text { are too } \\
\text { expensive } \\
\text { (yes-no [1- } \\
0] \text { ) }\end{array}$ & $\begin{array}{l}\text { Pay out-of- } \\
\text { pocket (oop) } \\
\text { medical cost } \\
\text { or medication } \\
\text { (yes-no [1-0]) }\end{array}$ & $\begin{array}{l}\text { Oop } \\
\text { expenses } \\
\text { paid by } \\
\text { relatives } \\
\text { (yes-no [1- } \\
0] \text { ) }\end{array}$ \\
\hline Wave & $\begin{array}{l}0.254 \\
(0.141)\end{array}$ & $\begin{array}{l}0.233 \\
(0.140)\end{array}$ & $\begin{array}{l}-0.023 \\
(0.231)\end{array}$ & $\begin{array}{l}-0.593 \\
(0.138) * *\end{array}$ & $\begin{array}{l}-0.515 \\
(0.102)^{* *}\end{array}$ & $\begin{array}{l}-0.116 \\
(0.089)\end{array}$ & $\begin{array}{l}-0.076 \\
(0.112)\end{array}$ \\
\hline Valladolid & $\begin{array}{l}-0.116 \\
(0.092)\end{array}$ & $\begin{array}{l}-0.123 \\
(0.090)\end{array}$ & $\begin{array}{l}-0.014 \\
(0.160)\end{array}$ & $\begin{array}{l}-0.180 \\
(0.100)\end{array}$ & $\begin{array}{l}-0.136 \\
(0.068) *\end{array}$ & $\begin{array}{l}-0.112 \\
(0.060)\end{array}$ & $\begin{array}{l}-0.369 \\
(0.071)^{* *}\end{array}$ \\
\hline Valladolid wave 2 & $\begin{array}{l}-0.149 \\
(0.121)\end{array}$ & $\begin{array}{l}-0.156 \\
(0.117)\end{array}$ & $\begin{array}{l}-0.157 \\
(0.244)\end{array}$ & $\begin{array}{l}0.112 \\
(0.137)\end{array}$ & $\begin{array}{l}-0.189 \\
(0.093) *\end{array}$ & $\begin{array}{l}0.016 \\
(0.078)\end{array}$ & $\begin{array}{l}-0.406 \\
(0.095) * *\end{array}$ \\
\hline Age & $\begin{array}{l}-0.114 \\
(0.090)\end{array}$ & $\begin{array}{l}-0.105 \\
(0.095)\end{array}$ & $\begin{array}{l}-0.105 \\
(0.158)\end{array}$ & $\begin{array}{l}0.063 \\
(0.119)\end{array}$ & $\begin{array}{l}0.110 \\
(0.084)\end{array}$ & $\begin{array}{l}0.004 \\
(0.070)\end{array}$ & $\begin{array}{l}0.024 \\
(0.078)\end{array}$ \\
\hline Age squared & 0.001 & 0.001 & 0.001 & -0.000 & -0.001 & -0.000 & -0.000 \\
\hline
\end{tabular}




\begin{tabular}{|c|c|c|c|c|c|c|c|}
\hline \multirow{3}{*}{ Gender (male=1) } & $(0.001)$ & $(0.001)$ & $(0.001)$ & $(0.001)$ & $(0.001)$ & $(0.000)$ & $(0.000)$ \\
\hline & -0.153 & -0.143 & 0.063 & 0.003 & -0.112 & -0.151 & -0.275 \\
\hline & $(0.068)^{*}$ & $(0.067)^{*}$ & $(0.131)$ & $(0.066)$ & $(0.052)^{*}$ & $(0.044)^{* *}$ & $(0.052)^{* *}$ \\
\hline \multirow[t]{2}{*}{ Speaks Maya } & -0.020 & -0.026 & -0.207 & -0.455 & -0.251 & -0.039 & 0.095 \\
\hline & $(0.107)$ & $(0.107)$ & $(0.182)$ & $(0.098)^{* *}$ & $(0.076)^{* *}$ & $(0.070)$ & $(0.081)$ \\
\hline \multirow[t]{2}{*}{ Reads/writes Spanish } & 0.255 & 0.247 & 0.255 & -0.109 & -0.149 & -0.002 & -0.158 \\
\hline & $(0.106)^{*}$ & $(0.104)^{*}$ & $(0.190)$ & $(0.107)$ & $(0.073)^{*}$ & $(0.064)$ & $(0.075) *$ \\
\hline \multirow[t]{2}{*}{ Lives alone } & 0.182 & 0.182 & -0.235 & 0.073 & -0.049 & -0.181 & -0.214 \\
\hline & $(0.125)$ & $(0.124)$ & $(0.244)$ & $(0.116)$ & $(0.095)$ & $(0.078)^{*}$ & $(0.094) *$ \\
\hline \multirow[t]{2}{*}{ household size } & -0.022 & -0.018 & -0.048 & -0.022 & 0.017 & -0.006 & 0.037 \\
\hline & $(0.022)$ & $(0.022)$ & $(0.034)$ & $(0.018)$ & $(0.014)$ & $(0.011)$ & $(0.013)^{* *}$ \\
\hline \multirow[t]{2}{*}{ Incomplete primary } & 0.182 & 0.188 & -0.233 & -0.239 & -0.145 & 0.067 & 0.065 \\
\hline & $(0.078)^{*}$ & $(0.075)^{*}$ & (0.139) & $(0.081)^{* *}$ & $(0.059) *$ & $(0.051)$ & $(0.061)$ \\
\hline \multirow[t]{2}{*}{ Primary } & 0.409 & 0.431 & -0.030 & -0.482 & -0.119 & 0.154 & -0.016 \\
\hline & $(0.109)^{* *}$ & $(0.104)^{* *}$ & $(0.203)$ & $(0.142)^{* *}$ & $(0.093)$ & $(0.076)^{*}$ & $(0.098)$ \\
\hline \multirow[t]{2}{*}{ Couple } & 0.111 & 0.125 & 4.179 & 0.065 & 0.003 & -0.200 & -0.406 \\
\hline & $(0.152)$ & $(0.147)$ & (6.527) & $(0.146)$ & $(0.108)$ & $(0.099) *$ & $(0.117)^{* *}$ \\
\hline \multirow[t]{2}{*}{ Divorced/Separated } & 0.215 & 0.230 & 4.107 & -0.206 & 0.301 & 0.238 & 0.120 \\
\hline & $(0.208)$ & $(0.203)$ & (6.473) & $(0.243)$ & $(0.164)$ & $(0.141)$ & $(0.174)$ \\
\hline \multirow[t]{2}{*}{ Widow } & -0.070 & -0.044 & 4.014 & -0.035 & -0.094 & -0.107 & -0.043 \\
\hline & $(0.151)$ & $(0.147)$ & (6.497) & $(0.145)$ & $(0.106)$ & $(0.098)$ & $(0.115)$ \\
\hline \multirow[t]{2}{*}{ Constant } & 3.475 & & -2.111 & -2.952 & -4.315 & 0.028 & -1.632 \\
\hline & (3.682) & & $(0.000)$ & $(4.816)$ & (3.385) & $(2.830)$ & (3.202) \\
\hline Observations & 4008 & 4006 & 4008 & 4003 & 4006 & 4007 & 4006 \\
\hline F-test education & 14.21 & 17.18 & 3.23 & 14.73 & 6.06 & 4.29 & 1.58 \\
\hline Prob $>F$ & 0.001 & 0.000 & 0.199 & 0.381 & 0.048 & 0.117 & 0.453 \\
\hline F-test age & 6.124 & 6.349 & 1.086 & 1.932 & 5.522 & 0.141 & 16.883 \\
\hline Prob $>F$ & 0.047 & 0.042 & 0.581 & 0.001 & 0.063 & 0.932 & 0.000 \\
\hline age parabola min/max & 90.797 & 91.767 & 77.461 & 72.699 & 75.511 & 122.690 & 274.805 \\
\hline
\end{tabular}

NOTE: We do not show in this table all the control variables included in the regressions. The reference categories for the control variables are wave 1, Motul, female, do not speak Maya, do not read write a message in Spanish, no schooling, single, and the missing category for respondent income and wealth. The model for the number of outpatient procedures could not be estimated due to the few numbers of observations.

SOURCE: Baseline and first follow-up ENCAHEY, 2008 and 2009.

** = significant at $5 \%$ level of confidence. $*=$ significant at $10 \%$ level of confidence 
Table 6e: Treatment regressions

\begin{tabular}{|c|c|c|c|c|c|c|c|c|}
\hline & $\begin{array}{l}\text { Oop } \\
\text { expenses } \\
\text { paid by } \\
\text { elderly } \\
\text { eligible (yes- } \\
\text { no [1-0]) }\end{array}$ & $\begin{array}{l}\text { Serious } \\
\text { health } \\
\text { problem but } \\
\text { did not go to } \\
\text { the doctor } \\
\text { (yes-no[1-0]) }\end{array}$ & $\begin{array}{l}\text { Did not go to } \\
\text { the doctor } \\
\text { because of } \\
\text { money (yes- } \\
\text { no[1-0]) }\end{array}$ & $\begin{array}{l}\text { Relatives or } \\
\text { friends pay } \\
\text { your } \\
\text { expenses } \\
\text { (not true, } \\
\text { sometimes } \\
\text { true, often } \\
\text { true, al }\end{array}$ & $\begin{array}{l}\text { Feel a } \\
\text { burden on } \\
\text { your family } \\
\text { or friends } \\
\text { (not true, } \\
\text { sometimes } \\
\text { true, often } \\
\text { true, a }\end{array}$ & $\begin{array}{l}\text { Activities you } \\
\text { used to do but } \\
\text { can't do } \\
\text { because of } \\
\text { lack of money } \\
\text { (yes-no[1-0]) }\end{array}$ & $\begin{array}{l}\text { Donation to } \\
\text { the church } \\
\text { but can't do } \\
\text { due to } \\
\text { money (yes- } \\
\text { no[1-0]) }\end{array}$ & $\begin{array}{l}\text { Donation to } \\
\text { local } \\
\text { parties but } \\
\text { can't do due } \\
\text { to money } \\
\text { (yes-no[1- } \\
0] \text { ) }\end{array}$ \\
\hline Wave & $\begin{array}{l}-0.091 \\
(0.101)\end{array}$ & $\begin{array}{l}-0.590 \\
(0.121)^{* *}\end{array}$ & $\begin{array}{l}-0.655 \\
(0.135)^{* *}\end{array}$ & $\begin{array}{l}0.109 \\
(0.076)\end{array}$ & $\begin{array}{l}-0.365 \\
(0.147)^{*}\end{array}$ & $\begin{array}{l}-0.500 \\
(0.111)^{* *}\end{array}$ & $\begin{array}{l}-0.489 \\
(0.216) *\end{array}$ & $\begin{array}{l}-0.563 \\
(0.424)\end{array}$ \\
\hline Valladolid & $\begin{array}{l}0.188 \\
(0.069)^{* *}\end{array}$ & $\begin{array}{l}-0.213 \\
(0.074)^{* *}\end{array}$ & $\begin{array}{l}-0.123 \\
(0.085)\end{array}$ & $\begin{array}{l}-0.116 \\
(0.057)^{*}\end{array}$ & $\begin{array}{l}0.154 \\
(0.105)\end{array}$ & $\begin{array}{l}-0.272 \\
(0.070) * *\end{array}$ & $\begin{array}{l}-0.119 \\
(0.144)\end{array}$ & $\begin{array}{l}-0.125 \\
(0.209)\end{array}$ \\
\hline Valladolid wave 2 & $\begin{array}{l}0.314 \\
(0.087)^{* *}\end{array}$ & $\begin{array}{l}-0.046 \\
(0.112)\end{array}$ & $\begin{array}{l}-0.044 \\
(0.133)\end{array}$ & $\begin{array}{l}-0.039 \\
(0.065)\end{array}$ & $\begin{array}{l}0.381 \\
(0.150)^{*}\end{array}$ & $\begin{array}{l}0.013 \\
(0.100)\end{array}$ & $\begin{array}{l}0.082 \\
(0.236)\end{array}$ & $\begin{array}{l}-0.001 \\
(0.429)\end{array}$ \\
\hline Age & $\begin{array}{l}0.047 \\
(0.074)\end{array}$ & $\begin{array}{l}0.030 \\
(0.085)\end{array}$ & $\begin{array}{l}-0.015 \\
(0.102)\end{array}$ & $\begin{array}{l}0.101 \\
(0.065)\end{array}$ & $\begin{array}{l}0.042 \\
(0.118)\end{array}$ & $\begin{array}{l}-0.145 \\
(0.077)\end{array}$ & $\begin{array}{l}0.108 \\
(0.230)\end{array}$ & $\begin{array}{l}-0.033 \\
(0.234)\end{array}$ \\
\hline Age squared & $\begin{array}{l}-0.000 \\
(0.000)\end{array}$ & $\begin{array}{l}-0.000 \\
(0.001)\end{array}$ & $\begin{array}{l}0.000 \\
(0.001)\end{array}$ & $\begin{array}{l}-0.000 \\
(0.000)\end{array}$ & $\begin{array}{l}-0.000 \\
(0.001)\end{array}$ & $\begin{array}{l}0.001 \\
(0.000)\end{array}$ & $\begin{array}{l}-0.001 \\
(0.001)\end{array}$ & $\begin{array}{l}0.000 \\
(0.001)\end{array}$ \\
\hline Gender (male=1) & $\begin{array}{l}0.100 \\
(0.049)^{*}\end{array}$ & $\begin{array}{l}-0.171 \\
(0.058)^{* *}\end{array}$ & $\begin{array}{l}-0.211 \\
(0.067)^{* *}\end{array}$ & $\begin{array}{l}-0.210 \\
(0.039)^{* *}\end{array}$ & $\begin{array}{l}-0.018 \\
(0.080)\end{array}$ & $\begin{array}{l}0.017 \\
(0.050)\end{array}$ & $\begin{array}{l}-0.443 \\
(0.116)^{* *}\end{array}$ & $\begin{array}{l}0.219 \\
(0.189)\end{array}$ \\
\hline Speaks Maya & $\begin{array}{l}-0.183 \\
(0.077)^{*}\end{array}$ & $\begin{array}{l}-0.123 \\
(0.084)\end{array}$ & $\begin{array}{l}-0.201 \\
(0.093)^{*}\end{array}$ & $\begin{array}{l}0.155 \\
(0.064)^{*}\end{array}$ & $\begin{array}{l}-0.163 \\
(0.121)\end{array}$ & $\begin{array}{l}-0.041 \\
(0.078)\end{array}$ & $\begin{array}{l}-0.232 \\
(0.148)\end{array}$ & $\begin{array}{l}-0.208 \\
(0.205)\end{array}$ \\
\hline Reads/writes Spanish & $\begin{array}{l}0.128 \\
(0.074)\end{array}$ & $\begin{array}{l}-0.196 \\
(0.083)^{*}\end{array}$ & $\begin{array}{l}-0.144 \\
(0.098)\end{array}$ & $\begin{array}{l}-0.013 \\
(0.056)\end{array}$ & $\begin{array}{l}-0.047 \\
(0.114)\end{array}$ & $\begin{array}{l}-0.038 \\
(0.077)\end{array}$ & $\begin{array}{l}0.010 \\
(0.172)\end{array}$ & $\begin{array}{l}0.072 \\
(0.246)\end{array}$ \\
\hline Lives alone & $\begin{array}{l}0.030 \\
(0.090)\end{array}$ & $\begin{array}{l}0.040 \\
(0.101)\end{array}$ & $\begin{array}{l}-0.051 \\
(0.119)\end{array}$ & $\begin{array}{l}-0.154 \\
(0.074)^{*}\end{array}$ & $\begin{array}{l}0.264 \\
(0.132)^{*}\end{array}$ & $\begin{array}{l}-0.053 \\
(0.089)\end{array}$ & $\begin{array}{l}0.238 \\
(0.171)\end{array}$ & $\begin{array}{l}0.204 \\
(0.329)\end{array}$ \\
\hline household size & $\begin{array}{l}-0.043 \\
(0.015)^{* *}\end{array}$ & $\begin{array}{l}-0.001 \\
(0.014)\end{array}$ & $\begin{array}{l}0.003 \\
(0.016)\end{array}$ & $\begin{array}{l}0.058 \\
(0.012)^{* *}\end{array}$ & $\begin{array}{l}0.030 \\
(0.019)\end{array}$ & $\begin{array}{l}0.024 \\
(0.014)\end{array}$ & $\begin{array}{l}-0.021 \\
(0.030)\end{array}$ & $\begin{array}{l}0.022 \\
(0.042)\end{array}$ \\
\hline Incomplete primary & $\begin{array}{l}0.050 \\
(0.057)\end{array}$ & $\begin{array}{l}0.121 \\
(0.069)\end{array}$ & $\begin{array}{l}-0.009 \\
(0.081)\end{array}$ & $\begin{array}{l}-0.079 \\
(0.046)\end{array}$ & $\begin{array}{l}-0.033 \\
(0.093)\end{array}$ & $\begin{array}{l}0.046 \\
(0.060)\end{array}$ & $\begin{array}{l}0.107 \\
(0.141)\end{array}$ & $\begin{array}{l}-0.223 \\
(0.247)\end{array}$ \\
\hline
\end{tabular}




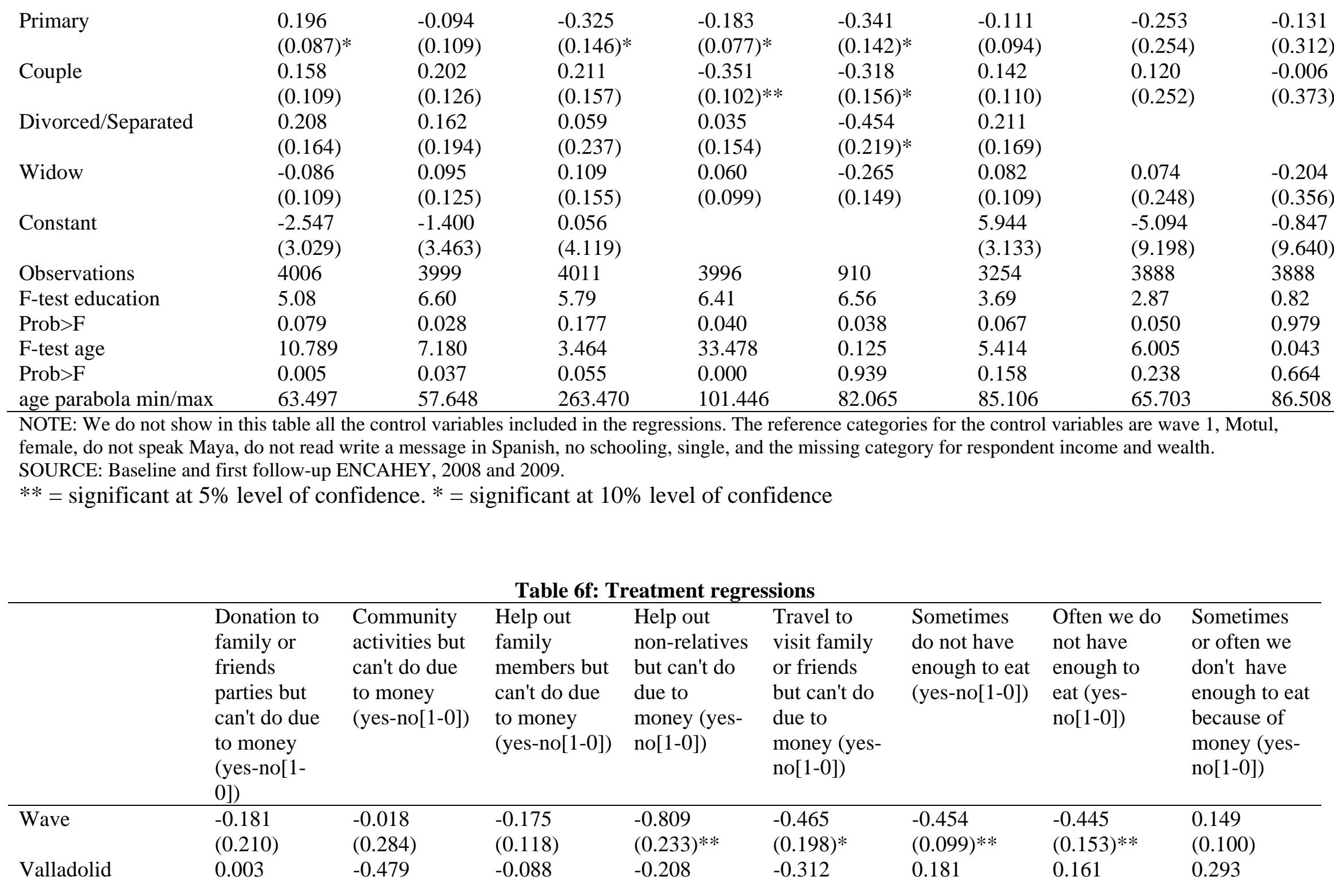




\begin{tabular}{|c|c|c|c|c|c|c|c|c|}
\hline \multirow{3}{*}{ Valladolid wave 2} & $(0.122)$ & $(0.187)^{*}$ & $(0.079)$ & $(0.133)$ & $(0.111)^{* *}$ & $(0.071)^{*}$ & $(0.106)$ & $(0.071)^{* *}$ \\
\hline & 0.112 & -0.235 & 0.134 & 0.160 & -0.078 & -0.060 & 0.242 & 0.084 \\
\hline & $(0.216)$ & $(0.284)$ & $(0.108)$ & $(0.235)$ & (0.194) & $(0.093)$ & $(0.154)$ & $(0.089)$ \\
\hline \multirow[t]{2}{*}{ Age } & -0.030 & -0.071 & -0.065 & 0.113 & 0.131 & 0.027 & 0.013 & -0.001 \\
\hline & $(0.182)$ & $(0.153)$ & $(0.083)$ & $(0.184)$ & $(0.143)$ & $(0.090)$ & $(0.105)$ & $(0.086)$ \\
\hline \multirow[t]{2}{*}{ Age squared } & 0.000 & 0.000 & 0.000 & -0.001 & -0.001 & -0.000 & -0.000 & -0.000 \\
\hline & $(0.001)$ & $(0.001)$ & $(0.001)$ & $(0.001)$ & $(0.001)$ & $(0.001)$ & $(0.001)$ & $(0.001)$ \\
\hline \multirow[t]{2}{*}{ Gender (male=1) } & -0.123 & 0.120 & 0.072 & 0.092 & 0.019 & 0.026 & 0.003 & -0.017 \\
\hline & $(0.106)$ & $(0.118)$ & $(0.053)$ & $(0.098)$ & $(0.081)$ & (0.039) & $(0.058)$ & $(0.038)$ \\
\hline \multirow[t]{2}{*}{ Speaks Maya } & 0.140 & -0.278 & -0.052 & -0.265 & -0.158 & -0.196 & -0.077 & 0.378 \\
\hline & $(0.146)$ & $(0.165)$ & $(0.086)$ & $(0.135)$ & $(0.112)$ & $(0.076)^{* *}$ & $(0.113)$ & $(0.080)^{* *}$ \\
\hline \multirow{2}{*}{$\begin{array}{l}\text { Reads/writes } \\
\text { Spanish }\end{array}$} & 0.244 & 0.251 & -0.062 & -0.121 & 0.117 & -0.174 & -0.066 & -0.014 \\
\hline & $(0.140)$ & (0.189) & $(0.084)$ & $(0.153)$ & $(0.119)$ & $(0.070)^{*}$ & $(0.105)$ & $(0.069)$ \\
\hline \multirow[t]{2}{*}{ Lives alone } & 0.345 & 0.193 & -0.075 & 0.081 & 0.040 & 0.026 & 0.221 & 0.153 \\
\hline & $(0.172) *$ & $(0.205)$ & $(0.100)$ & $(0.169)$ & $(0.145)$ & $(0.085)$ & $(0.124)$ & $(0.083)$ \\
\hline \multirow[t]{2}{*}{ household size } & 0.056 & 0.012 & 0.015 & -0.004 & 0.034 & 0.007 & 0.007 & 0.013 \\
\hline & $(0.022)^{*}$ & $(0.027)$ & $(0.014)$ & $(0.027)$ & (0.019) & $(0.015)$ & $(0.020)$ & $(0.014)$ \\
\hline \multirow[t]{2}{*}{ Incomplete primary } & -0.217 & 0.012 & 0.025 & 0.017 & 0.002 & -0.148 & -0.209 & -0.164 \\
\hline & $(0.119)$ & $(0.144)$ & $(0.063)$ & $(0.123)$ & $(0.104)$ & $(0.054)^{* *}$ & $(0.090)^{*}$ & $(0.054)^{* *}$ \\
\hline \multirow[t]{2}{*}{ Primary } & -0.072 & -0.090 & -0.280 & -0.070 & 0.065 & -0.568 & -0.304 & -0.481 \\
\hline & $(0.166)$ & $(0.214)$ & $(0.106)^{* *}$ & $(0.200)$ & $(0.148)$ & $(0.097)^{* *}$ & $(0.134)^{*}$ & $(0.091)^{* *}$ \\
\hline \multirow[t]{2}{*}{ Couple } & 0.303 & 0.169 & -0.013 & -0.282 & -0.058 & -0.189 & 0.097 & -0.103 \\
\hline & $(0.284)$ & $(0.280)$ & $(0.121)$ & $(0.172)$ & $(0.161)$ & $(0.117)$ & $(0.160)$ & $(0.105)$ \\
\hline \multirow[t]{2}{*}{ Divorced/Separated } & 0.412 & 0.192 & 0.057 & 0.033 & 0.157 & -0.177 & 0.183 & -0.034 \\
\hline & $(0.347)$ & $(0.388)$ & $(0.202)$ & $(0.260)$ & $(0.233)$ & $(0.156)$ & $(0.232)$ & $(0.147)$ \\
\hline \multirow[t]{2}{*}{ Widow } & 0.219 & -0.039 & -0.078 & -0.320 & -0.174 & -0.308 & -0.074 & -0.243 \\
\hline & $(0.282)$ & $(0.275)$ & (0.119) & $(0.177)$ & $(0.164)$ & $(0.113)^{* *}$ & $(0.155)$ & $(0.100)^{*}$ \\
\hline \multirow[t]{2}{*}{ Constant } & -0.372 & 1.512 & 2.218 & -5.152 & -6.108 & -0.954 & -2.137 & -0.593 \\
\hline & (7.252) & (6.331) & (3.390) & (7.435) & (5.772) & (3.611) & (4.313) & (3.476) \\
\hline Observations & 4011 & 4011 & 4011 & 4011 & 4011 & 4011 & 4011 & 4011 \\
\hline F-test education & 3.59 & 0.28 & 9.54 & 0.26 & 0.24 & 34.66 & 7.18 & 29.08 \\
\hline Prob $>F$ & 0.166 & 0.522 & 0.003 & 0.880 & 0.224 & 0.000 & 0.028 & 0.000 \\
\hline F-test age & 7.748 & 1.300 & 11.858 & 3.360 & 2.993 & 2.893 & 0.022 & 4.034 \\
\hline Prob $>F$ & 0.021 & 0.869 & 0.008 & 0.186 & 0.885 & 0.235 & 0.989 & 0.133 \\
\hline
\end{tabular}


NOTE: We do not show in this table all the control variables included in the regressions. The reference categories for the control variables are wave 1, Motul, female, do not speak Maya, do not read write a message in Spanish, no schooling, single, and the missing category for respondent income and wealth. SOURCE: Baseline and first follow-up ENCAHEY, 2008 and 2009.

$* *=$ significant at $5 \%$ level of confidence. $*=$ significant at $10 \%$ level of confidence

Table 6g: Treatment regressions

\begin{tabular}{|c|c|c|c|c|c|c|c|c|}
\hline & $\begin{array}{l}\text { Often } \\
\text { worried to } \\
\text { run out of } \\
\text { food last } \\
\text { three months } \\
\text { (never- } \\
\text { always [1-4]) }\end{array}$ & $\begin{array}{l}\text { Run out of } \\
\text { food and } \\
\text { money not } \\
\text { enough } \\
\text { (never- } \\
\text { always [1-4]) }\end{array}$ & $\begin{array}{l}\text { Skip or cut } \\
\text { meals } \\
\text { (never- } \\
\text { always [1-4]) }\end{array}$ & $\begin{array}{l}\text { Often eat } \\
\text { less than you } \\
\text { felt you } \\
\text { should } \\
\text { (never- } \\
\text { always [1-4]) }\end{array}$ & $\begin{array}{l}\text { Often hungry } \\
\text { (never- } \\
\text { always [1-4]) }\end{array}$ & $\begin{array}{l}\text { Not eat all } \\
\text { day (never- } \\
\text { always [1-4]) }\end{array}$ & $\begin{array}{l}\text { Food from } \\
\text { charity } \\
\text { (never- } \\
\text { always [1-4]) }\end{array}$ & $\begin{array}{l}\text { Spend on } \\
\text { food at home } \\
\text { last week }\end{array}$ \\
\hline \multirow[t]{2}{*}{ Wave } & -0.509 & -0.371 & -0.468 & -0.382 & -0.583 & -0.491 & -0.302 & 34.036 \\
\hline & $(0.085)^{* *}$ & $(0.088)^{* *}$ & $(0.090)^{* *}$ & $(0.091)^{* *}$ & $(0.106)^{* *}$ & $(0.122)^{* *}$ & $(0.144)^{*}$ & (24.454) \\
\hline \multirow[t]{2}{*}{ Valladolid } & -0.001 & -0.175 & -0.082 & -0.086 & -0.213 & -0.253 & -0.207 & -21.176 \\
\hline & $(0.061)$ & $(0.061)^{* *}$ & $(0.063)$ & $(0.062)$ & $(0.070)^{* *}$ & $(0.081)^{* *}$ & $(0.112)$ & (15.137) \\
\hline \multirow[t]{2}{*}{ Valladolid wave 2} & 0.168 & -0.233 & -0.122 & -0.195 & -0.217 & -0.427 & -0.358 & 19.624 \\
\hline & $(0.079)^{*}$ & $(0.083)^{* *}$ & $(0.087)$ & $(0.088)^{*}$ & $(0.103)^{*}$ & $(0.122)^{* *}$ & $(0.162)^{*}$ & (22.521) \\
\hline \multirow[t]{2}{*}{ Age } & -0.134 & -0.187 & -0.118 & -0.219 & -0.217 & -0.173 & 0.014 & 14.357 \\
\hline & $(0.077)$ & $(0.081)^{*}$ & $(0.085)$ & $(0.074)^{* *}$ & $(0.092) *$ & (0.109) & $(0.118)$ & (18.337) \\
\hline \multirow[t]{2}{*}{ Age squared } & 0.001 & 0.001 & 0.001 & 0.001 & 0.001 & 0.001 & -0.000 & -0.090 \\
\hline & $(0.000)$ & $(0.001)^{*}$ & $(0.001)$ & $(0.000) * *$ & $(0.001)^{*}$ & $(0.001)$ & $(0.001)$ & $(0.113)$ \\
\hline \multirow[t]{2}{*}{ Gender (male=1) } & 0.069 & 0.081 & 0.071 & 0.099 & 0.147 & 0.105 & -0.002 & -17.678 \\
\hline & $(0.035) *$ & $(0.036)^{*}$ & $(0.036)$ & $(0.037) * *$ & $(0.044) * *$ & $(0.052)^{*}$ & $(0.067)$ & (11.733) \\
\hline \multirow[t]{2}{*}{ Speaks Maya } & -0.130 & -0.152 & -0.083 & -0.074 & -0.135 & -0.133 & -0.313 & 13.314 \\
\hline & $(0.066) *$ & $(0.067)^{*}$ & $(0.069)$ & $(0.071)$ & $(0.075)$ & $(0.084)$ & $(0.111)^{* *}$ & (17.906) \\
\hline \multirow{2}{*}{$\begin{array}{l}\text { Reads/writes } \\
\text { Spanish }\end{array}$} & -0.153 & -0.151 & -0.219 & -0.108 & -0.151 & -0.189 & -0.177 & 23.641 \\
\hline & $(0.062) *$ & $(0.061)^{*}$ & $(0.063) * *$ & $(0.062)$ & $(0.074)^{*}$ & $(0.081)^{*}$ & $(0.109)$ & (17.439) \\
\hline
\end{tabular}




\begin{tabular}{|c|c|c|c|c|c|c|c|c|}
\hline Lives alone & $\begin{array}{l}-0.060 \\
(0.075)\end{array}$ & $\begin{array}{l}-0.088 \\
(0.077)\end{array}$ & $\begin{array}{l}-0.103 \\
(0.079)\end{array}$ & $\begin{array}{l}-0.103 \\
(0.080)\end{array}$ & $\begin{array}{l}0.086 \\
(0.091)\end{array}$ & $\begin{array}{l}0.109 \\
(0.101)\end{array}$ & $\begin{array}{l}0.331 \\
(0.137)^{*}\end{array}$ & $\begin{array}{l}-134.979 \\
(19.573)^{* *}\end{array}$ \\
\hline \multirow[t]{2}{*}{ household size } & 0.014 & -0.003 & 0.017 & 0.009 & 0.001 & -0.009 & -0.013 & 28.061 \\
\hline & $(0.012)$ & $(0.012)$ & $(0.012)$ & $(0.012)$ & $(0.015)$ & $(0.017)$ & $(0.024)$ & $(2.840)^{* *}$ \\
\hline \multirow[t]{2}{*}{ Incomplete primary } & -0.148 & -0.231 & -0.110 & -0.171 & -0.185 & -0.219 & -0.106 & 34.847 \\
\hline & $(0.050)^{* *}$ & $(0.051)^{* *}$ & $(0.053) *$ & $(0.052)^{* *}$ & $(0.062) * *$ & $(0.067)^{* *}$ & $(0.098)$ & $(13.666)^{*}$ \\
\hline \multirow[t]{2}{*}{ Primary } & -0.523 & -0.669 & -0.547 & -0.664 & -0.691 & -0.519 & -0.334 & 178.675 \\
\hline & $(0.081)^{* *}$ & $(0.090)^{* *}$ & $(0.088)^{* *}$ & $(0.087)^{* *}$ & $(0.110)^{* *}$ & $(0.119)^{* *}$ & $(0.170)^{*}$ & $(20.287) * *$ \\
\hline \multirow[t]{2}{*}{ Couple } & 0.170 & 0.102 & 0.000 & 0.056 & 0.003 & -0.120 & 0.231 & 43.576 \\
\hline & $(0.110)$ & $(0.092)$ & $(0.106)$ & $(0.104)$ & $(0.119)$ & $(0.129)$ & $(0.196)$ & $(25.752)$ \\
\hline \multirow[t]{2}{*}{ Divorced/Separated } & 0.176 & 0.236 & 0.130 & 0.116 & 0.252 & 0.361 & -0.164 & 69.720 \\
\hline & $(0.155)$ & $(0.153)$ & $(0.167)$ & $(0.166)$ & $(0.161)$ & $(0.178) *$ & $(0.285)$ & (39.318) \\
\hline \multirow[t]{2}{*}{ Widow } & 0.129 & 0.035 & -0.090 & -0.054 & -0.042 & -0.172 & 0.132 & 75.805 \\
\hline & $(0.108)$ & $(0.088)$ & $(0.103)$ & $(0.100)$ & $(0.111)$ & $(0.123)$ & $(0.176)$ & $(25.796) * *$ \\
\hline Constant & & & & & & & & $\begin{array}{l}-259.730 \\
(742.484)\end{array}$ \\
\hline Observations & 3624 & 3624 & 3619 & 3623 & 3624 & 3625 & 3630 & 3041 \\
\hline F-test education & 42.14 & 58.02 & 38.80 & 58.85 & 39.93 & 21.89 & 4.01 & 40.65 \\
\hline Prob $>F$ & 0.000 & 0.053 & 0.377 & 0.012 & 0.027 & 0.092 & 0.261 & 0.686 \\
\hline F-test age & 4.422 & 5.860 & 1.952 & 8.889 & 7.188 & 4.782 & 2.685 & 0.377 \\
\hline Prob $>F$ & 0.110 & 0.000 & 0.000 & 0.000 & 0.000 & 0.000 & 0.135 & 0.000 \\
\hline age parabola & 78.486 & 82.407 & 80.578 & 81.181 & 83.306 & 84.744 & 434.251 & 79.375 \\
\hline
\end{tabular}

NOTE: We do not show in this table all the control variables included in the regressions. The reference categories for the control variables are wave 1, Motul, female, do not speak Maya, do not read write a message in Spanish, no schooling, single, and the missing category for respondent income and wealth.

SOURCE: Baseline and first follow-up ENCAHEY, 2008 and 2009.

$* *=$ significant at $5 \%$ level of confidence. $*=$ significant at $10 \%$ level of confidence

Table 6h: Treatment regressions

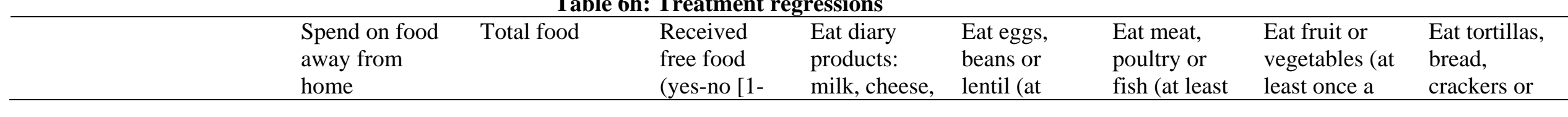




\begin{tabular}{|c|c|c|c|c|c|c|c|c|}
\hline & & & 0]) & $\begin{array}{l}\text { yogurt (at } \\
\text { least once a } \\
\text { day, at least } \\
\text { once a w }\end{array}$ & $\begin{array}{l}\text { least once a } \\
\text { day, at least } \\
\text { once a week, } \\
\text { several ti }\end{array}$ & $\begin{array}{l}\text { once a day, } \\
\text { at least once } \\
\text { a week, } \\
\text { several ti }\end{array}$ & $\begin{array}{l}\text { day, at least } \\
\text { once a week, } \\
\text { several time }\end{array}$ & $\begin{array}{l}\text { other cereals } \\
\text { (at least once a } \\
\text { day, at least o }\end{array}$ \\
\hline Wave & $\begin{array}{l}-51.567 \\
(125.097)\end{array}$ & $\begin{array}{l}38.118 \\
(36.006)\end{array}$ & $\begin{array}{l}0.473 \\
(0.130)^{* *}\end{array}$ & $\begin{array}{l}-0.042 \\
(0.070)\end{array}$ & $\begin{array}{l}0.294 \\
(0.082) * *\end{array}$ & $\begin{array}{l}0.132 \\
(0.080)\end{array}$ & $\begin{array}{l}-0.022 \\
(0.073)\end{array}$ & $\begin{array}{l}-0.601 \\
(0.146)^{* *}\end{array}$ \\
\hline Valladolid & $\begin{array}{l}-65.655 \\
(79.183)\end{array}$ & $\begin{array}{l}-35.839 \\
(22.292)\end{array}$ & $\begin{array}{l}-0.252 \\
(0.091)^{* *}\end{array}$ & $\begin{array}{l}0.145 \\
(0.053)^{* *}\end{array}$ & $\begin{array}{l}0.223 \\
(0.058)^{* *}\end{array}$ & $\begin{array}{l}0.055 \\
(0.058)\end{array}$ & $\begin{array}{l}0.017 \\
(0.057)\end{array}$ & $\begin{array}{l}-0.199 \\
(0.099) *\end{array}$ \\
\hline Valladolid wave 2 & $\begin{array}{l}-356.556 \\
(119.377)^{* *}\end{array}$ & $\begin{array}{l}11.244 \\
(33.166)\end{array}$ & $\begin{array}{l}-0.257 \\
(0.116)^{*}\end{array}$ & $\begin{array}{l}-0.045 \\
(0.059)\end{array}$ & $\begin{array}{l}0.005 \\
(0.071)\end{array}$ & $\begin{array}{l}-0.002 \\
(0.069)\end{array}$ & $\begin{array}{l}-0.001 \\
(0.066)\end{array}$ & $\begin{array}{l}0.102 \\
(0.146)\end{array}$ \\
\hline Age & $\begin{array}{l}103.863 \\
(102.730)\end{array}$ & $\begin{array}{l}15.354 \\
(26.994)\end{array}$ & $\begin{array}{l}0.187 \\
(0.103)\end{array}$ & $\begin{array}{l}-0.044 \\
(0.074)\end{array}$ & $\begin{array}{l}0.002 \\
(0.056)\end{array}$ & $\begin{array}{l}0.002 \\
(0.061)\end{array}$ & $\begin{array}{l}-0.054 \\
(0.066)\end{array}$ & $\begin{array}{l}0.046 \\
(0.087)\end{array}$ \\
\hline Age squared & $\begin{array}{l}-0.721 \\
(0.638)\end{array}$ & $\begin{array}{l}-0.100 \\
(0.166)\end{array}$ & $\begin{array}{l}-0.001 \\
(0.001)\end{array}$ & $\begin{array}{l}0.000 \\
(0.000)\end{array}$ & $\begin{array}{l}0.000 \\
(0.000)\end{array}$ & $\begin{array}{l}-0.000 \\
(0.000)\end{array}$ & $\begin{array}{l}0.000 \\
(0.000)\end{array}$ & $\begin{array}{l}-0.000 \\
(0.001)\end{array}$ \\
\hline Gender (male=1) & $\begin{array}{l}42.153 \\
(61.599)\end{array}$ & $\begin{array}{l}-22.273 \\
(17.282)\end{array}$ & $\begin{array}{l}0.038 \\
(0.051)\end{array}$ & $\begin{array}{l}0.280 \\
(0.040)^{* *}\end{array}$ & $\begin{array}{l}-0.155 \\
(0.036)^{* *}\end{array}$ & $\begin{array}{l}0.118 \\
(0.038)^{* *}\end{array}$ & $\begin{array}{l}0.175 \\
(0.036)^{* *}\end{array}$ & $\begin{array}{l}0.001 \\
(0.070)\end{array}$ \\
\hline Speaks Maya & $\begin{array}{l}136.874 \\
(94.790)\end{array}$ & $\begin{array}{l}26.699 \\
(26.405)\end{array}$ & $\begin{array}{l}0.196 \\
(0.100)\end{array}$ & $\begin{array}{l}0.245 \\
(0.057) * *\end{array}$ & $\begin{array}{l}0.109 \\
(0.066)\end{array}$ & $\begin{array}{l}0.256 \\
(0.065)^{* *}\end{array}$ & $\begin{array}{l}0.113 \\
(0.061)\end{array}$ & $\begin{array}{l}-0.311 \\
(0.096)^{* *}\end{array}$ \\
\hline Reads/writes Spanish & $\begin{array}{l}-221.788 \\
(90.773)^{*}\end{array}$ & $\begin{array}{l}4.146 \\
(25.690)\end{array}$ & $\begin{array}{l}0.115 \\
(0.088)\end{array}$ & $\begin{array}{l}-0.300 \\
(0.054)^{* *}\end{array}$ & $\begin{array}{l}0.080 \\
(0.062)\end{array}$ & $\begin{array}{l}-0.209 \\
(0.058) * *\end{array}$ & $\begin{array}{l}-0.184 \\
(0.057)^{* *}\end{array}$ & $\begin{array}{l}-0.298 \\
(0.101)^{* *}\end{array}$ \\
\hline Lives alone & $\begin{array}{l}-2.564 \\
(105.834)\end{array}$ & $\begin{array}{l}-149.879 \\
(28.862)^{* *}\end{array}$ & $\begin{array}{l}0.290 \\
(0.103)^{* *}\end{array}$ & $\begin{array}{l}0.093 \\
(0.074)\end{array}$ & $\begin{array}{l}0.028 \\
(0.068)\end{array}$ & $\begin{array}{l}0.130 \\
(0.073)\end{array}$ & $\begin{array}{l}0.142 \\
(0.069) *\end{array}$ & $\begin{array}{l}0.070 \\
(0.119)\end{array}$ \\
\hline household size & $\begin{array}{l}6.258 \\
(14.500)\end{array}$ & $\begin{array}{l}30.389 \\
(4.179) * *\end{array}$ & $\begin{array}{l}-0.144 \\
(0.026)^{* *}\end{array}$ & $\begin{array}{l}0.039 \\
(0.010) * *\end{array}$ & $\begin{array}{l}-0.024 \\
(0.010)^{*}\end{array}$ & $\begin{array}{l}-0.026 \\
(0.009) * *\end{array}$ & $\begin{array}{l}0.012 \\
(0.010)\end{array}$ & $\begin{array}{l}-0.008 \\
(0.019)\end{array}$ \\
\hline Incomplete primary & $\begin{array}{l}123.515 \\
(73.877)\end{array}$ & $\begin{array}{l}54.544 \\
(20.114)^{* * *}\end{array}$ & $\begin{array}{l}-0.140 \\
(0.069) *\end{array}$ & $\begin{array}{l}-0.191 \\
(0.045)^{* *}\end{array}$ & $\begin{array}{l}0.066 \\
(0.045)\end{array}$ & $\begin{array}{l}-0.105 \\
(0.043)^{*}\end{array}$ & $\begin{array}{l}-0.188 \\
(0.042)^{* *}\end{array}$ & $\begin{array}{l}-0.128 \\
(0.080)\end{array}$ \\
\hline Primary & $\begin{array}{l}538.477 \\
(100.552)^{* *}\end{array}$ & $\begin{array}{l}257.247 \\
(29.959)^{* *}\end{array}$ & $\begin{array}{l}-0.233 \\
(0.111)^{*}\end{array}$ & $\begin{array}{l}-0.568 \\
(0.078) * *\end{array}$ & $\begin{array}{l}0.047 \\
(0.064)\end{array}$ & $\begin{array}{l}-0.403 \\
(0.071)^{* *}\end{array}$ & $\begin{array}{l}-0.564 \\
(0.073) * *\end{array}$ & $\begin{array}{l}-0.098 \\
(0.125)\end{array}$ \\
\hline Couple & $\begin{array}{l}-102.796 \\
(126.459)\end{array}$ & $\begin{array}{l}52.529 \\
(38.145)\end{array}$ & $\begin{array}{l}-0.160 \\
(0.141)\end{array}$ & $\begin{array}{l}0.015 \\
(0.097)\end{array}$ & $\begin{array}{l}-0.044 \\
(0.097)\end{array}$ & $\begin{array}{l}0.027 \\
(0.103)\end{array}$ & $\begin{array}{l}0.056 \\
(0.093)\end{array}$ & $\begin{array}{l}0.027 \\
(0.145)\end{array}$ \\
\hline Divorced/Separated & $\begin{array}{l}-233.247 \\
(210.026)\end{array}$ & $\begin{array}{l}60.656 \\
(57.987)\end{array}$ & $\begin{array}{l}-0.061 \\
(0.188)\end{array}$ & $\begin{array}{l}-0.049 \\
(0.142)\end{array}$ & $\begin{array}{l}0.088 \\
(0.140)\end{array}$ & $\begin{array}{l}-0.056 \\
(0.140)\end{array}$ & $\begin{array}{l}-0.013 \\
(0.128)\end{array}$ & $\begin{array}{l}0.270 \\
(0.208)\end{array}$ \\
\hline Widow & $\begin{array}{l}-146.154 \\
(127.049)\end{array}$ & $\begin{array}{l}73.970 \\
(38.214)\end{array}$ & $\begin{array}{l}-0.084 \\
(0.133)\end{array}$ & $\begin{array}{l}-0.151 \\
(0.098)\end{array}$ & $\begin{array}{l}0.024 \\
(0.094)\end{array}$ & $\begin{array}{l}-0.030 \\
(0.102)\end{array}$ & $\begin{array}{l}-0.014 \\
(0.092)\end{array}$ & $\begin{array}{l}0.006 \\
(0.146)\end{array}$ \\
\hline Constant & $-4,939.638$ & -251.117 & -8.399 & & & & & \\
\hline
\end{tabular}




\begin{tabular}{llllllll} 
& $(4,132.779)$ & $(1,093.175)$ & $(4.203)^{*}$ & & & \\
Observations & 3929 & 3028 & 3621 & 4002 & 4000 & 4002 & 4002 \\
F-test education & 15.45 & 38.17 & 6.08 & 55.76 & 2.13 & 32.17 & 62.09 \\
Prob>F & 0.000 & 0.689 & 0.048 & 0.000 & 0.346 & 0.891 & 0.668 \\
F-test age & 3.649 & 0.372 & 4.414 & 14.328 & 2.874 & 0.231 & 0.806 \\
Prob>F & 0.026 & 0.000 & 0.110 & 0.001 & 0.238 & 0.000 & 0.488 \\
age parabola min/max & 71.992 & 76.956 & 84.281 & 115.006 & -49.100 & 43.519 & 83.339 \\
\hline
\end{tabular}

NOTE: We do not show in this table all the control variables included in the regressions. The reference categories for the control variables are wave 1, Motul, female, do not speak Maya, do not read write a message in Spanish, no schooling, single, and the missing category for respondent income and wealth.

SOURCE: Baseline and first follow-up ENCAHEY, 2008 and 2009.

$* *=$ significant at $5 \%$ level of confidence. $*$ = significant at $10 \%$ level of confidence

Table 6i: Treatment regressions

\begin{tabular}{|c|c|c|c|c|c|c|c|}
\hline & $\begin{array}{l}\text { Worked for } \\
\text { pay last } \\
\text { month (yes-no } \\
[1-0])\end{array}$ & $\begin{array}{l}\text { Immediate } \\
\text { recall } \\
\text { (number of } \\
\text { words) }\end{array}$ & $\begin{array}{l}\text { Delayed recall } \\
\text { (number of } \\
\text { words) }\end{array}$ & $\begin{array}{l}\text { Feel fear } \\
\text { someone } \\
\text { robbing you } \\
\text { (never, } \\
\text { sometimes, } \\
\text { usually, always } \\
{[1-4] \text { ) }}\end{array}$ & $\begin{array}{l}\text { Feel fear } \\
\text { someone close } \\
\text { to you will } \\
\text { take your } \\
\text { money (never, } \\
\text { sometimes, } \\
\text { usually, }\end{array}$ & $\begin{array}{l}\text { Money in a } \\
\text { safe place } \\
\text { (yes-no [1- } \\
0] \text { ) }\end{array}$ & $\begin{array}{l}\text { Feel verbally } \\
\text { or physically } \\
\text { abused (never, } \\
\text { sometimes, } \\
\text { usually, always } \\
{[1-4] \text { ) }}\end{array}$ \\
\hline Wave & $\begin{array}{l}-0.017 \\
(0.101)\end{array}$ & $\begin{array}{l}-0.020 \\
(0.064)\end{array}$ & $\begin{array}{l}0.063 \\
(0.071)\end{array}$ & $\begin{array}{l}-0.340 \\
(0.093) * *\end{array}$ & $\begin{array}{l}-0.211 \\
(0.122)\end{array}$ & $\begin{array}{l}-0.174 \\
(0.261)\end{array}$ & $\begin{array}{l}-0.140 \\
(0.126)\end{array}$ \\
\hline Valladolid & $\begin{array}{l}-0.125 \\
(0.075)\end{array}$ & $\begin{array}{l}0.077 \\
(0.049)\end{array}$ & $\begin{array}{l}0.105 \\
(0.050) *\end{array}$ & $\begin{array}{l}-0.214 \\
(0.065)^{* *}\end{array}$ & $\begin{array}{l}-0.136 \\
(0.076)\end{array}$ & $\begin{array}{l}-0.276 \\
(0.151)\end{array}$ & $\begin{array}{l}-0.109 \\
(0.087)\end{array}$ \\
\hline Valladolid wave 2 & $\begin{array}{l}-0.225 \\
(0.080) * *\end{array}$ & $\begin{array}{l}0.159 \\
(0.057)^{* *}\end{array}$ & $\begin{array}{l}0.282 \\
(0.060) * *\end{array}$ & $\begin{array}{l}0.059 \\
(0.085)\end{array}$ & $\begin{array}{l}0.157 \\
(0.108)\end{array}$ & $\begin{array}{l}-0.033 \\
(0.228)\end{array}$ & $\begin{array}{l}-0.017 \\
(0.125)\end{array}$ \\
\hline Age & $\begin{array}{l}-0.180 \\
(0.110)\end{array}$ & $\begin{array}{l}-0.007 \\
(0.071)\end{array}$ & $\begin{array}{l}-0.087 \\
(0.066)\end{array}$ & $\begin{array}{l}-0.060 \\
(0.091)\end{array}$ & $\begin{array}{l}-0.041 \\
(0.114)\end{array}$ & $\begin{array}{l}0.214 \\
(0.214)\end{array}$ & $\begin{array}{l}0.015 \\
(0.106)\end{array}$ \\
\hline Age squared & $\begin{array}{l}0.001 \\
(0.001)\end{array}$ & $\begin{array}{l}-0.000 \\
(0.000)\end{array}$ & $\begin{array}{l}0.000 \\
(0.000)\end{array}$ & $\begin{array}{l}0.000 \\
(0.001)\end{array}$ & $\begin{array}{l}0.000 \\
(0.001)\end{array}$ & $\begin{array}{l}-0.001 \\
(0.001)\end{array}$ & $\begin{array}{l}-0.000 \\
(0.001)\end{array}$ \\
\hline Gender (male=1) & $\begin{array}{l}1.030 \\
(0.072)^{* *}\end{array}$ & $\begin{array}{l}-0.310 \\
(0.038)^{* *}\end{array}$ & $\begin{array}{l}-0.308 \\
(0.039)^{* *}\end{array}$ & $\begin{array}{l}-0.150 \\
(0.046)^{* *}\end{array}$ & $\begin{array}{l}-0.130 \\
(0.057)^{*}\end{array}$ & $\begin{array}{l}-0.105 \\
(0.108)\end{array}$ & $\begin{array}{l}-0.197 \\
(0.070)^{* *}\end{array}$ \\
\hline Speaks Maya & -0.001 & -0.198 & -0.122 & -0.088 & -0.006 & -0.153 & -0.136 \\
\hline
\end{tabular}




$\begin{array}{llllllll} & (0.087) & (0.053)^{* *} & (0.058)^{*} & (0.070) & (0.089) & (0.162) & (0.098) \\ \text { Reads/writes Spanish } & -0.126 & 0.283 & 0.250 & -0.078 & 0.030 & 0.129 & 0.052 \\ & (0.078) & (0.051)^{* *} & (0.052)^{* *} & (0.070) & (0.085) & (0.160) & (0.101) \\ \text { Lives alone } & 0.234 & 0.008 & -0.070 & 0.041 & 0.162 & 0.142 & 0.260 \\ & (0.113)^{*} & (0.066) & (0.067) & (0.083) & (0.097) & (0.179) & (0.110)^{*} \\ \text { household size } & -0.005 & -0.022 & -0.021 & -0.029 & -0.014 & -0.033 & 0.028 \\ & (0.017) & (0.010)^{*} & (0.010)^{*} & (0.013)^{*} & (0.015) & (0.035) & (0.018) \\ \text { Incomplete primary } & 0.042 & 0.302 & 0.290 & 0.059 & -0.023 & 0.051 & 0.085 \\ & (0.070) & (0.044)^{* *} & (0.045)^{* *} & (0.055) & (0.062) & (0.126) & (0.080) \\ \text { Primary } & 0.053 & 0.745 & 0.637 & 0.048 & -0.264 & -0.216 & -0.154 \\ & (0.111) & (0.073)^{* *} & (0.073)^{* *} & (0.083) & (0.105)^{*} & (0.208) & (0.124) \\ \text { Couple } & -0.263 & 0.053 & 0.016 & 0.042 & -0.043 & -0.263 & 0.147 \\ & (0.139) & (0.089) & (0.094) & (0.121) & (0.119) & (0.185) & (0.153) \\ \text { Divorced/Separated } & -0.099 & 0.052 & -0.080 & -0.066 & -0.143 & -0.647 & 0.450 \\ & (0.203) & (0.127) & (0.127) & (0.174) & (0.177) & (0.402) & (0.201)^{*} \\ \text { Widow } & -0.398 & -0.195 & -0.181 & -0.006 & -0.143 & -0.271 & 0.040 \\ & (0.138)^{* *} & (0.088)^{*} & (0.093) & (0.121) & (0.119) & (0.181) & (0.152) \\ \text { Constant } & 8.168 & & & & -10.177 & (8.593) & 3250 \\ & (4.391) & & & & 3253 \\ \text { Observations } & 4006 & 4011 & 4011 & 3253 & 3251 & 2.05 & 5.01 \\ \text { F-test education } & 0.41 & 111.05 & 85.44 & 1.16 & 6.92 & 0.359 & 0.082 \\ \text { Prob>F } & 0.813 & 0.000 & 0.000 & 0.559 & 0.325 & 2.399 & 0.154 \\ \text { F-test age } & 93.268 & 170.094 & 164.673 & 3.420 & 2.250 & 0.301 \\ \text { Prob>F } & 0.000 & 0.000 & 0.000 & 0.181 & 0.031 & 0.301 \\ \text { age parabola min/max } & 116.606 & -15.407 & 169.817 & 71.979 & 68.310 & 84.657 & 70.739\end{array}$

NOTE: We do not show in this table all the control variables included in the regressions. The reference categories for the control variables are wave 1 , Motul, female, do not speak Maya, do not read write a message in Spanish, no schooling, single, and the missing category for respondent income and wealth. SOURCE: Baseline and first follow-up ENCAHEY, 2008 and 2009.

$* *=$ significant at $5 \%$ level of confidence. $*=$ significant at $10 \%$ level of confidence

\section{Table 7a: Interaction Effects}

\begin{tabular}{llllll}
\hline $\begin{array}{l}\text { Self-reported } \\
\text { health }\end{array}$ & $\begin{array}{l}\text { Subjective } \\
\text { mortality }\end{array}$ & $\begin{array}{l}\text { Feel sad, blue } \\
\text { or depressed }\end{array}$ & $\begin{array}{l}\text { Satisfied } \\
\text { relation fam. }\end{array}$ & $\begin{array}{l}\text { Satisfied hh } \\
\text { income (very }\end{array}$ & $\begin{array}{l}\text { Satisfied } \\
\text { social }\end{array}$ \\
\hline
\end{tabular}




\begin{tabular}{|c|c|c|c|c|c|c|c|c|}
\hline & $\begin{array}{l}\text { (excellent, } \\
\text { very good, } \\
\text { good, fair, } \\
\text { poor [1-5]) }\end{array}$ & $\begin{array}{l}\text { expectation } \\
\text { (chances to } \\
\text { leave at least } \\
10 \text { years more } \\
\text { [1-100 }\end{array}$ & $\begin{array}{l}\text { for } 2 \text { weeks } \\
\text { or more } \\
\text { during the } \\
\text { last } 3 \text { months } \\
\text { (yes-no }\end{array}$ & $\begin{array}{l}\text { members } \\
\text { (very } \\
\text { satisfied-very } \\
\text { unsatisfied } \\
{[1-5] \text { ) }}\end{array}$ & $\begin{array}{l}\text { satisfied-very } \\
\text { unsatisfied } \\
[1-5])\end{array}$ & $\begin{array}{l}\text { contacts (very } \\
\text { satisfied-very } \\
\text { unsatisfied } \\
{[1-5] \text { ) }}\end{array}$ & $\begin{array}{l}\text { satisfied-very } \\
\text { unsatisfied } \\
[1-5])\end{array}$ & $\begin{array}{l}\text { satisfied-very } \\
\text { unsatisfied } \\
[1-5])\end{array}$ \\
\hline \multirow[t]{2}{*}{ Wave } & -0.412 & 0.414 & -0.531 & -0.183 & -0.252 & -0.145 & -0.267 & -0.085 \\
\hline & $(0.076)^{* *}$ & $(2.414)$ & $(0.098) * *$ & $(0.086)^{*}$ & $(0.084)^{* *}$ & $(0.093)$ & $(0.084)^{* *}$ & $(0.082)$ \\
\hline \multirow[t]{2}{*}{ Valladolid } & 0.144 & -4.375 & -0.209 & -0.001 & 0.032 & -0.017 & -0.017 & 0.044 \\
\hline & $(0.055) * *$ & $(1.577)^{* *}$ & $(0.067) * *$ & $(0.063)$ & $(0.062)$ & $(0.065)$ & $(0.062)$ & $(0.059)$ \\
\hline \multirow{2}{*}{$\begin{array}{l}\text { Valladolid } \\
\text { wave } 2\end{array}$} & 0.121 & -2.242 & -0.015 & 0.077 & -0.076 & 0.111 & 0.150 & -0.124 \\
\hline & $(0.083)$ & $(2.916)$ & $(0.112)$ & $(0.100)$ & $(0.096)$ & $(0.108)$ & $(0.100)$ & $(0.093)$ \\
\hline \multirow{2}{*}{$\begin{array}{l}\text { Effect for } \\
\text { income } \\
\text { quartile } 1\end{array}$} & 0.097 & -1.592 & 0.013 & 0.187 & 0.104 & 0.199 & 0.063 & 0.061 \\
\hline & $(0.108)$ & $(4.061)$ & $(0.141)$ & $(0.123)$ & $(0.118)$ & $(0.130)$ & $(0.124)$ & $(0.109)$ \\
\hline \multirow{2}{*}{$\begin{array}{l}\text { Effect for } \\
\text { income } \\
\text { quartile } 2\end{array}$} & 0.084 & -4.999 & 0.028 & -0.146 & 0.015 & -0.022 & -0.045 & 0.081 \\
\hline & $(0.115)$ & (3.623) & $(0.148)$ & $(0.134)$ & $(0.124)$ & $(0.126)$ & $(0.127)$ & $(0.120)$ \\
\hline \multirow{2}{*}{$\begin{array}{l}\text { Effect for } \\
\text { income } \\
\text { quartile } 3\end{array}$} & 0.079 & 3.148 & -0.163 & -0.043 & -0.026 & -0.053 & -0.247 & 0.036 \\
\hline & $(0.118)$ & (3.988) & $(0.154)$ & $(0.136)$ & $(0.136)$ & $(0.128)$ & $(0.142)$ & $(0.122)$ \\
\hline \multirow{2}{*}{$\begin{array}{l}\text { Effect for } \\
\text { income } \\
\text { quartile } 4\end{array}$} & 0.078 & -1.673 & 0.041 & 0.042 & 0.161 & 0.108 & -0.022 & 0.247 \\
\hline & $(0.113)$ & (3.589) & $(0.152)$ & $(0.139)$ & $(0.129)$ & $(0.132)$ & $(0.128)$ & $(0.124)^{*}$ \\
\hline \multirow{2}{*}{$\begin{array}{l}\text { Effect on } \\
\text { males }\end{array}$} & -0.061 & 0.616 & -0.048 & 0.009 & -0.065 & -0.148 & -0.085 & -0.114 \\
\hline & $(0.076)$ & $(2.521)$ & $(0.098)$ & $(0.089)$ & $(0.085)$ & $(0.087)$ & $(0.085)$ & $(0.081)$ \\
\hline Constant & & $\begin{array}{l}308.603 \\
(78.959)^{* *}\end{array}$ & $\begin{array}{l}-3.506 \\
(3.250)\end{array}$ & & & & & \\
\hline Observations & 4007 & 2405 & 3271 & 3258 & 3253 & 3248 & 3257 & 3261 \\
\hline F-test SES & 0.03 & 1.13 & 1.76 & 5.08 & 2.04 & 3.91 & 4.09 & 2.75 \\
\hline
\end{tabular}


interactions

Prob>F

0.999

0.336

0.623

0.166

0.563

0.271

0.252

0.433

NOTE: We do not show in this table all the control variables included in the regressions. The reference categories for the control variables are wave 1, Motul, female, do not speak Maya, do not read write a message in Spanish, no schooling, single, and the missing category for respondent income and wealth. SOURCE: Baseline and first follow-up ENCAHEY, 2008 and 2009.

$* *=$ significant at $5 \%$ level of confidence. $*=$ significant at $10 \%$ level of confidence

Table 7b: Interaction Effects

\begin{tabular}{|c|c|c|c|c|c|c|c|c|}
\hline & $\begin{array}{l}\text { Satisfied life } \\
\text { in general } \\
\text { (very } \\
\text { satisfied-very } \\
\text { unsatisfied } \\
\text { [1-5]) }\end{array}$ & $\begin{array}{l}\text { Number of } \\
\text { acute } \\
\text { conditions }\end{array}$ & $\begin{array}{l}\text { Often pain } \\
\text { (yes-no [1-0]) }\end{array}$ & $\begin{array}{l}\text { How strong is } \\
\text { the pain } \\
\text { (mild, } \\
\text { moderate, } \\
\text { severe [1-3]) }\end{array}$ & $\begin{array}{l}\text { Number of } \\
\text { chronic } \\
\text { conditions }\end{array}$ & $\begin{array}{l}\text { Number of } \\
\text { ADL's }\end{array}$ & $\begin{array}{l}\text { Number of } \\
\text { IADL's }\end{array}$ & $\begin{array}{l}\text { Smokes now } \\
\text { (yes-no [1-0]) }\end{array}$ \\
\hline Wave & $\begin{array}{l}-0.164 \\
(0.093)\end{array}$ & $\begin{array}{l}-0.144 \\
(0.100)\end{array}$ & $\begin{array}{l}-0.327 \\
(0.084)^{* *}\end{array}$ & $\begin{array}{l}-0.273 \\
(0.072)^{* *}\end{array}$ & $\begin{array}{l}-0.044 \\
(0.070)\end{array}$ & $\begin{array}{l}-0.424 \\
(0.067)^{* *}\end{array}$ & $\begin{array}{l}-0.367 \\
(0.078)^{* *}\end{array}$ & $\begin{array}{l}-0.103 \\
(0.139)\end{array}$ \\
\hline Valladolid & $\begin{array}{l}0.045 \\
(0.066)\end{array}$ & $\begin{array}{l}-0.061 \\
(0.072)\end{array}$ & $\begin{array}{l}-0.023 \\
(0.062)\end{array}$ & $\begin{array}{l}-0.078 \\
(0.053)\end{array}$ & $\begin{array}{l}0.147 \\
(0.053)^{* *}\end{array}$ & $\begin{array}{l}0.081 \\
(0.051)\end{array}$ & $\begin{array}{l}-0.070 \\
(0.056)\end{array}$ & $\begin{array}{l}0.007 \\
(0.123)\end{array}$ \\
\hline \multirow[t]{2}{*}{$\begin{array}{l}\text { Valladolid } \\
\text { wave } 2\end{array}$} & -0.074 & -0.151 & 0.195 & 0.014 & -0.026 & 0.075 & 0.128 & -0.052 \\
\hline & $(0.103)$ & (0.119) & $(0.097)^{*}$ & $(0.082)$ & $(0.072)$ & (0.072) & (0.089) & (0.208) \\
\hline \multirow[t]{2}{*}{$\begin{array}{l}\text { Effect for } \\
\text { income } \\
\text { quartile } 1\end{array}$} & 0.176 & 0.065 & 0.105 & 0.125 & 0.155 & -0.018 & -0.035 & 0.040 \\
\hline & (0.119) & (0.154) & $(0.128)$ & (0.108) & (0.095) & (0.094) & $(0.107)$ & $(0.240)$ \\
\hline \multirow[t]{2}{*}{$\begin{array}{l}\text { Effect for } \\
\text { income } \\
\text { quartile } 2\end{array}$} & 0.004 & 0.061 & 0.123 & 0.102 & 0.018 & 0.067 & -0.206 & 0.323 \\
\hline & $(0.120)$ & $(0.156)$ & $(0.128)$ & $(0.103)$ & (0.104) & (0.095) & $(0.119)$ & $(0.237)$ \\
\hline
\end{tabular}




\begin{tabular}{|c|c|c|c|c|c|c|c|c|}
\hline $\begin{array}{l}\text { Effect for } \\
\text { income } \\
\text { quartile } 3\end{array}$ & $(0.126)$ & $(0.175)$ & -0.122 & -0.080 & -0.066 & $(0.104) *$ & -0.148 & $(0.277)$ \\
\hline \multirow{2}{*}{$\begin{array}{l}\text { Effect for } \\
\text { income } \\
\text { quartile } 4\end{array}$} & 0.189 & 0.018 & 0.091 & 0.118 & -0.027 & 0.004 & -0.237 & 0.097 \\
\hline & $(0.137)$ & $(0.173)$ & $(0.131)$ & $(0.114)$ & $(0.111)$ & $(0.103)$ & $(0.125)$ & $(0.226)$ \\
\hline $\begin{array}{l}\text { Effect on } \\
\text { males }\end{array}$ & -0.106 & -0.048 & -0.111 & -0.072 & 0.061 & -0.063 & -0.017 & 0.024 \\
\hline Constant & $(0.088)$ & $(0.108)$ & $\begin{array}{l}(0.089) \\
-3.151 \\
(2.671)\end{array}$ & $(0.074)$ & $(0.071)$ & $(0.069)$ & $(0.081)$ & $\begin{array}{l}(0.177) \\
1.010 \\
(6.575)\end{array}$ \\
\hline Observations & 3257 & 4011 & 4006 & 4003 & 4011 & 3769 & 4002 & 4008 \\
\hline $\begin{array}{l}\text { F-test SES } \\
\text { interactions }\end{array}$ & 2.74 & 0.08 & 3.09 & 3.13 & 3.72 & 7.52 & 2.75 & 1.50 \\
\hline Prob $>F$ & 0.433 & 0.994 & 0.378 & 0.372 & 0.293 & 0.057 & 0.432 & 0.682 \\
\hline
\end{tabular}

NOTE: We do not show in this table all the control variables included in the regressions. The reference categories for the control variables are wave 1, Motul, female, do not speak Maya, do not read write a message in Spanish, no schooling, single, and the missing category for respondent income and wealth. SOURCE: Baseline and first follow-up ENCAHEY, 2008 and 2009.

$* *=$ significant at $5 \%$ level of confidence. $*=$ significant at $10 \%$ level of confidence

Table 7c: Interaction Effects

\begin{tabular}{|c|c|c|c|c|c|c|c|c|}
\hline & $\begin{array}{l}\text { Number of } \\
\text { cigarettes in a } \\
\text { day }\end{array}$ & $\begin{array}{l}\text { Drink } \\
\text { alcoholic } \\
\text { beverages } \\
\text { (yes-no [1-0]) }\end{array}$ & $\begin{array}{l}\text { Number of } \\
\text { days a week } \\
\text { drinks } \\
\text { alcoholic } \\
\text { beverages }\end{array}$ & $\begin{array}{l}\text { Number of } \\
\text { drinks per } \\
\text { day }\end{array}$ & $\begin{array}{l}\text { Visited a } \\
\text { doctor (yes- } \\
\text { no [1-0]) }\end{array}$ & $\begin{array}{l}\text { Number of } \\
\text { doctor visits }\end{array}$ & $\begin{array}{l}\text { Visited a folk } \\
\text { healer (yes- } \\
\text { no [1-0]) }\end{array}$ & $\begin{array}{l}\text { Number of } \\
\text { folk healer } \\
\text { visits }\end{array}$ \\
\hline Wave & $\begin{array}{l}-1.906 \\
(1.800)\end{array}$ & $\begin{array}{l}0.371 \\
(0.092) * *\end{array}$ & $\begin{array}{l}0.141 \\
(0.120)\end{array}$ & $\begin{array}{l}0.970 \\
(0.622)\end{array}$ & $\begin{array}{l}0.220 \\
(0.089) *\end{array}$ & $\begin{array}{l}0.166 \\
(0.083) *\end{array}$ & $\begin{array}{l}-0.216 \\
(0.168)\end{array}$ & $\begin{array}{l}-0.187 \\
(0.162)\end{array}$ \\
\hline Valladolid & $\begin{array}{l}0.923 \\
(1.103)\end{array}$ & $\begin{array}{l}0.101 \\
(0.065)\end{array}$ & $\begin{array}{l}-0.161 \\
(0.080)^{*}\end{array}$ & $\begin{array}{l}-1.152 \\
(0.403) * *\end{array}$ & $\begin{array}{l}0.150 \\
(0.061)^{*}\end{array}$ & $\begin{array}{l}0.132 \\
(0.057)^{*}\end{array}$ & $\begin{array}{l}-0.336 \\
(0.116)^{* *}\end{array}$ & $\begin{array}{l}-0.333 \\
(0.116) * *\end{array}$ \\
\hline
\end{tabular}




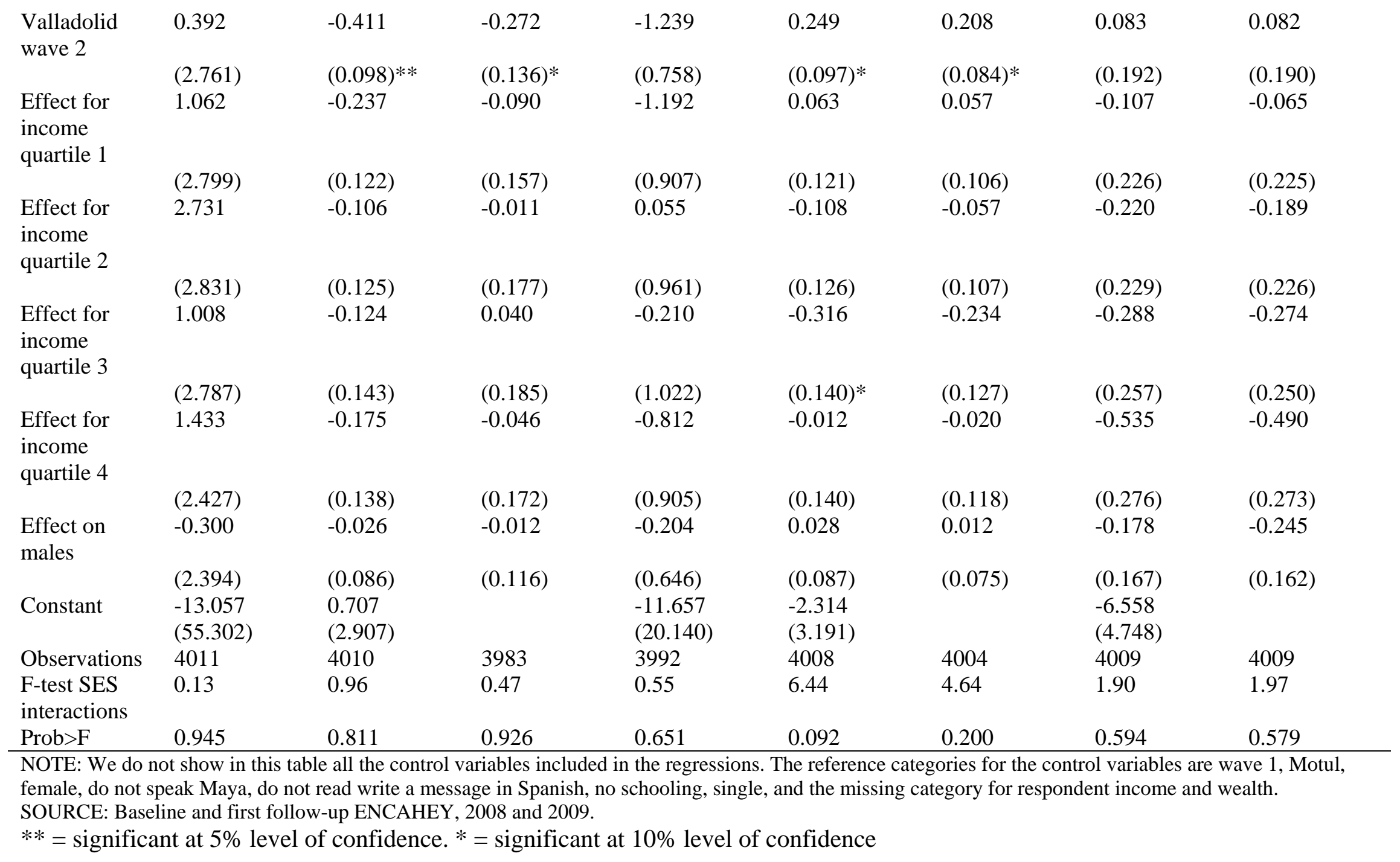


Table 7d: Interaction Effects

\begin{tabular}{|c|c|c|c|c|c|c|c|}
\hline & $\begin{array}{l}\text { Visited a } \\
\text { dentist (yes- } \\
\text { no [1-0]) }\end{array}$ & $\begin{array}{l}\text { Number of } \\
\text { dentist visits }\end{array}$ & $\begin{array}{l}\text { Outpatient } \\
\text { procedures } \\
\text { (yes-no [1-0]) }\end{array}$ & $\begin{array}{l}\text { Consulted a } \\
\text { pharmacist } \\
\text { (yes-no [1-0]) }\end{array}$ & $\begin{array}{l}\text { Bought no } \\
\text { medicines } \\
\text { because they } \\
\text { are too } \\
\text { expensive } \\
\text { (yes-no [1-0]) }\end{array}$ & $\begin{array}{l}\text { Pay out-of- } \\
\text { pocket (oop) } \\
\text { medical cost } \\
\text { or medication } \\
\text { (yes-no [1-0]) }\end{array}$ & $\begin{array}{l}\text { Oop expenses } \\
\text { paid by } \\
\text { relatives (yes- } \\
\text { no [1-0]) }\end{array}$ \\
\hline \multirow[t]{2}{*}{ Wave } & 0.243 & 0.215 & -0.017 & -0.612 & -0.513 & -0.121 & -0.064 \\
\hline & $(0.143)$ & $(0.142)$ & $(0.234)$ & $(0.140) * *$ & $(0.103) * *$ & $(0.089)$ & $(0.113)$ \\
\hline \multirow[t]{2}{*}{ Valladolid } & -0.150 & -0.155 & -0.044 & -0.204 & -0.151 & -0.118 & -0.349 \\
\hline & $(0.093)$ & $(0.092)$ & $(0.163)$ & $(0.102)^{*}$ & $(0.069) *$ & $(0.061)$ & $(0.072) * *$ \\
\hline \multirow{2}{*}{$\begin{array}{l}\text { Valladolid } \\
\text { wave } 2\end{array}$} & -0.269 & -0.253 & 0.274 & 0.318 & -0.088 & 0.162 & -0.376 \\
\hline & $(0.155)$ & $(0.151)$ & $(0.326)$ & $(0.178)$ & $(0.123)$ & $(0.099)$ & $(0.117)^{* *}$ \\
\hline \multirow{2}{*}{$\begin{array}{l}\text { Effect for } \\
\text { income } \\
\text { quartile } 1\end{array}$} & 0.084 & 0.091 & -0.698 & -0.075 & -0.094 & -0.163 & -0.021 \\
\hline & $(0.203)$ & (0.199) & $(0.449)$ & $(0.200)$ & $(0.159)$ & $(0.126)$ & $(0.149)$ \\
\hline \multirow{2}{*}{$\begin{array}{l}\text { Effect for } \\
\text { income } \\
\text { quartile } 2\end{array}$} & 0.277 & 0.296 & -0.737 & -0.046 & 0.074 & -0.221 & -0.151 \\
\hline & $(0.219)$ & $(0.214)$ & $(0.449)$ & $(0.190)$ & $(0.155)$ & $(0.127)$ & $(0.151)$ \\
\hline \multirow{2}{*}{$\begin{array}{l}\text { Effect for } \\
\text { income } \\
\text { quartile } 3\end{array}$} & 0.374 & 0.348 & -0.719 & -0.788 & 0.041 & -0.363 & -0.166 \\
\hline & $(0.189)^{*}$ & $(0.181)$ & $(0.443)$ & $(0.303) * *$ & $(0.183)$ & $(0.145)^{*}$ & $(0.175)$ \\
\hline \multirow{2}{*}{$\begin{array}{l}\text { Effect for } \\
\text { income } \\
\text { quartile } 4\end{array}$} & 0.259 & 0.274 & -0.390 & -0.488 & 0.067 & -0.052 & -0.063 \\
\hline & $(0.196)$ & $(0.186)$ & $(0.481)$ & $(0.288)$ & $(0.187)$ & (0.133) & $(0.166)$ \\
\hline \multirow{2}{*}{$\begin{array}{l}\text { Effect on } \\
\text { males }\end{array}$} & -0.093 & -0.143 & 0.020 & -0.162 & -0.286 & -0.061 & 0.038 \\
\hline & $(0.131)$ & $(0.125)$ & $(0.275)$ & $(0.142)$ & $(0.108) * *$ & $(0.084)$ & $(0.106)$ \\
\hline Constant & 3.020 & & -1.461 & -2.901 & -4.387 & 0.021 & -1.443 \\
\hline
\end{tabular}




\begin{tabular}{|c|c|c|c|c|c|c|c|}
\hline & (3.620) & & (6.637) & (4.995) & (3.386) & (2.829) & (3.214) \\
\hline Observations & 4008 & 4006 & 4008 & 4003 & 4006 & 4007 & 4006 \\
\hline $\begin{array}{l}\text { F-test SES } \\
\text { interactions }\end{array}$ & 1.58 & 1.40 & 0.54 & 7.14 & 1.04 & 3.60 & 0.85 \\
\hline Prob $>F$ & 0.664 & 0.704 & 0.909 & 0.068 & 0.791 & 0.308 & 0.839 \\
\hline
\end{tabular}

NOTE: We do not show in this table all the control variables included in the regressions. The reference categories for the control variables are wave 1 , Motul, female, do not speak Maya, do not read write a message in Spanish, no schooling, single, and the missing category for respondent income and wealth. The model for the number of outpatient procedures could not be estimated due to the few numbers of observations.

SOURCE: Baseline and first follow-up ENCAHEY, 2008 and 2009.

** = significant at $5 \%$ level of confidence. ${ }^{*}=$ significant at $10 \%$ level of confidence

Table 7e: Interaction Effects

\begin{tabular}{|c|c|c|c|c|c|c|c|c|}
\hline & $\begin{array}{l}\text { Oop expenses } \\
\text { paid by } \\
\text { elderly } \\
\text { eligible (yes- } \\
\text { no [1-0]) }\end{array}$ & $\begin{array}{l}\text { Serious } \\
\text { health } \\
\text { problem but } \\
\text { did not go to } \\
\text { the doctor } \\
\text { (yes-no[1-0]) }\end{array}$ & $\begin{array}{l}\text { Did not go to } \\
\text { the doctor } \\
\text { because of } \\
\text { money (yes- } \\
\text { no[1-0]) }\end{array}$ & $\begin{array}{l}\text { Relatives or } \\
\text { friends pay } \\
\text { your } \\
\text { expenses (not } \\
\text { true, } \\
\text { sometimes } \\
\text { true, often } \\
\text { true, al }\end{array}$ & $\begin{array}{l}\text { Feel a burden } \\
\text { on your } \\
\text { family or } \\
\text { friends (not } \\
\text { true, } \\
\text { sometimes } \\
\text { true, often } \\
\text { true, a }\end{array}$ & $\begin{array}{l}\text { Activities you } \\
\text { used to do but } \\
\text { can't do } \\
\text { because of } \\
\text { lack of } \\
\text { money (yes- } \\
\text { no[1-0]) }\end{array}$ & $\begin{array}{l}\text { Donation to } \\
\text { the church } \\
\text { but can't do } \\
\text { due to money } \\
\text { (yes-no[1-0]) }\end{array}$ & $\begin{array}{l}\text { Donation to } \\
\text { family or } \\
\text { friends } \\
\text { parties but } \\
\text { can't do due } \\
\text { to money } \\
\text { (yes-no[1-0]) }\end{array}$ \\
\hline Wave & $\begin{array}{l}-0.108 \\
(0.102)\end{array}$ & $\begin{array}{l}-0.577 \\
(0.122)^{* *}\end{array}$ & $\begin{array}{l}-0.639 \\
(0.136)^{* *}\end{array}$ & $\begin{array}{l}0.130 \\
(0.077)\end{array}$ & $\begin{array}{l}-0.375 \\
(0.147) *\end{array}$ & $\begin{array}{l}-0.482 \\
(0.111)^{* *}\end{array}$ & $\begin{array}{l}-0.477 \\
(0.219)^{*}\end{array}$ & $\begin{array}{l}-0.158 \\
(0.208)\end{array}$ \\
\hline Valladolid & $\begin{array}{l}0.162 \\
(0.070) *\end{array}$ & $\begin{array}{l}-0.224 \\
(0.075)^{* *}\end{array}$ & $\begin{array}{l}-0.129 \\
(0.087)\end{array}$ & $\begin{array}{l}-0.081 \\
(0.057)\end{array}$ & $\begin{array}{l}0.151 \\
(0.106)\end{array}$ & $\begin{array}{l}-0.276 \\
(0.070)^{* *}\end{array}$ & $\begin{array}{l}-0.124 \\
(0.145)\end{array}$ & $\begin{array}{l}-0.020 \\
(0.123)\end{array}$ \\
\hline $\begin{array}{l}\text { Valladolid } \\
\text { wave } 2\end{array}$ & $\begin{array}{l}0.465 \\
(0.116)^{* *}\end{array}$ & $\begin{array}{l}-0.036 \\
(0.145)\end{array}$ & $\begin{array}{l}0.109 \\
(0.168)\end{array}$ & $\begin{array}{l}-0.142 \\
(0.089)\end{array}$ & $\begin{array}{l}0.386 \\
(0.197) *\end{array}$ & $\begin{array}{l}-0.068 \\
(0.133)\end{array}$ & $\begin{array}{l}-0.036 \\
(0.288)\end{array}$ & $\begin{array}{l}0.324 \\
(0.280)\end{array}$ \\
\hline $\begin{array}{l}\text { Effect for } \\
\text { income } \\
\text { quartile } 1\end{array}$ & -0.099 & -0.155 & -0.383 & 0.073 & 0.185 & -0.007 & 0.434 & -0.487 \\
\hline Effect for & $\begin{array}{l}(0.145) \\
-0.140\end{array}$ & $\begin{array}{l}(0.187) \\
-0.033\end{array}$ & $\begin{array}{l}(0.222) \\
-0.220\end{array}$ & $\begin{array}{l}(0.117) \\
0.131\end{array}$ & $\begin{array}{l}(0.232) \\
-0.033\end{array}$ & $\begin{array}{l}(0.156) \\
0.146\end{array}$ & $(0.318)$ & $\begin{array}{l}(0.341) \\
-0.063\end{array}$ \\
\hline
\end{tabular}

quartile 2 


\begin{tabular}{|c|c|c|c|c|c|c|c|c|}
\hline & $(0.148)$ & $(0.175)$ & $(0.223)$ & $(0.120)$ & $(0.260)$ & $(0.157)$ & & $(0.337)$ \\
\hline \multirow{2}{*}{$\begin{array}{l}\text { Effect for } \\
\text { income } \\
\text { quartile } 3\end{array}$} & -0.249 & -0.149 & -0.248 & 0.305 & 0.119 & 0.103 & 0.056 & -0.557 \\
\hline & $(0.162)$ & $(0.218)$ & $(0.271)$ & $(0.127)^{*}$ & $(0.280)$ & $(0.171)$ & $(0.387)$ & $(0.435)$ \\
\hline \multirow{2}{*}{$\begin{array}{l}\text { Effect for } \\
\text { income } \\
\text { quartile } 4\end{array}$} & -0.020 & 0.124 & 0.373 & 0.087 & 0.194 & 0.201 & 0.766 & -0.374 \\
\hline & $(0.144)$ & $(0.195)$ & $(0.233)$ & $(0.127)$ & $(0.277)$ & $(0.162)$ & $(0.442)$ & $(0.423)$ \\
\hline \multirow{2}{*}{$\begin{array}{l}\text { Effect on } \\
\text { males }\end{array}$} & -0.151 & 0.042 & -0.163 & 0.035 & -0.170 & 0.018 & -0.233 & 0.007 \\
\hline & $(0.096)$ & $(0.122)$ & $(0.153)$ & $(0.075)$ & $(0.173)$ & $(0.107)$ & $(0.310)$ & $(0.245)$ \\
\hline \multirow[t]{2}{*}{ Constant } & -2.961 & -1.095 & 0.425 & & & 5.921 & -5.818 & -0.399 \\
\hline & $(3.001)$ & $(3.465)$ & $(4.125)$ & & & $(3.136)$ & $(9.211)$ & (7.383) \\
\hline Observations & 4006 & 3999 & 4011 & 3996 & 910 & 3254 & 3702 & 4011 \\
\hline $\begin{array}{l}\text { F-test SES } \\
\text { interactions }\end{array}$ & 1.67 & 1.91 & 8.93 & 3.23 & 0.75 & 1.45 & 2.31 & 1.68 \\
\hline Prob $>F$ & 0.643 & 0.592 & 0.030 & 0.357 & 0.861 & 0.694 & 0.315 & 0.641 \\
\hline \multicolumn{9}{|c|}{$\begin{array}{l}\text { NOTE: We do not show in this table all the control variables included in the regressions. The reference categories for the control variables are wave } 1 \text {, Motul, } \\
\text { female, do not speak Maya, do not read write a message in Spanish, no schooling, single, and the missing category for respondent income and wealth. } \\
\text { SOURCE: Baseline and first follow-up ENCAHEY, } 2008 \text { and } 2009 . \\
* *=\text { significant at } 5 \% \text { level of confidence. } *=\text { significant at } 10 \% \text { level of confidence }\end{array}$} \\
\hline \multicolumn{9}{|c|}{ Table 7f: Interaction Effects } \\
\hline & $\begin{array}{l}\text { Community } \\
\text { activities but } \\
\text { can't do due } \\
\text { to money } \\
\text { (yes-no[1-0]) }\end{array}$ & $\begin{array}{l}\text { Help out } \\
\text { family } \\
\text { members but } \\
\text { can't do due } \\
\text { to money } \\
\text { (yes-no[1-0]) }\end{array}$ & $\begin{array}{l}\text { Help out non- } \\
\text { relatives but } \\
\text { can't do due } \\
\text { to money } \\
\text { (yes-no[1-0]) }\end{array}$ & $\begin{array}{l}\text { Travel to visit } \\
\text { family or } \\
\text { friends but } \\
\text { can't do due } \\
\text { to money } \\
\text { (yes-no[1-0]) }\end{array}$ & $\begin{array}{l}\text { Sometimes } \\
\text { do not have } \\
\text { enough to eat } \\
\text { (yes-no[1-0]) }\end{array}$ & $\begin{array}{l}\text { Often we do } \\
\text { not have } \\
\text { enough to eat } \\
\text { (yes-no[1-0]) }\end{array}$ & $\begin{array}{l}\text { Sometimes or } \\
\text { often we } \\
\text { don't have } \\
\text { enough to eat } \\
\text { because of } \\
\text { money (yes- } \\
\text { no[1-0]) }\end{array}$ & $\begin{array}{l}\text { Often worried } \\
\text { to run out of } \\
\text { food last } \\
\text { three months } \\
\text { (never-always } \\
{[1-4] \text { ) }}\end{array}$ \\
\hline \multirow[t]{2}{*}{ Wave } & -0.025 & -0.157 & -0.783 & -0.455 & -0.459 & -0.440 & 0.157 & -0.511 \\
\hline & $(0.293)$ & $(0.119)$ & $(0.231)^{* *}$ & $(0.196)^{*}$ & $(0.100)^{* *}$ & $(0.154)^{* *}$ & $(0.100)$ & $(0.086)^{* *}$ \\
\hline Valladolid & -0.535 & -0.094 & -0.187 & -0.287 & 0.170 & 0.199 & 0.299 & -0.009 \\
\hline
\end{tabular}




\begin{tabular}{|c|c|c|c|c|c|c|c|c|}
\hline & $(0.199)^{* *}$ & $(0.079)$ & $(0.129)$ & $(0.112)^{*}$ & $(0.071)^{*}$ & $(0.107)$ & $(0.071)^{* *}$ & $(0.061)$ \\
\hline \multirow{2}{*}{$\begin{array}{l}\text { Valladolid } \\
\text { wave } 2\end{array}$} & -0.284 & -0.055 & 0.035 & 0.047 & 0.041 & 0.422 & 0.153 & 0.252 \\
\hline & $(0.362)$ & $(0.135)$ & $(0.286)$ & $(0.239)$ & $(0.119)$ & $(0.190) *$ & $(0.115)$ & $(0.103)^{*}$ \\
\hline \multirow{2}{*}{$\begin{array}{l}\text { Effect for } \\
\text { income } \\
\text { quartile } 1\end{array}$} & -0.019 & 0.267 & -0.455 & -0.331 & 0.016 & -0.305 & -0.013 & -0.028 \\
\hline & $(0.445)$ & $(0.160)$ & $(0.414)$ & $(0.278)$ & $(0.142)$ & $(0.221)$ & $(0.137)$ & $(0.127)$ \\
\hline \multirow{2}{*}{$\begin{array}{l}\text { Effect for } \\
\text { income } \\
\text { quartile } 2\end{array}$} & 0.473 & 0.155 & 0.396 & -0.083 & -0.018 & -0.050 & 0.088 & -0.033 \\
\hline & $(0.340)$ & $(0.160)$ & $(0.305)$ & $(0.284)$ & $(0.146)$ & $(0.231)$ & $(0.141)$ & $(0.131)$ \\
\hline \multirow{2}{*}{$\begin{array}{l}\text { Effect for } \\
\text { income } \\
\text { quartile } 3\end{array}$} & -0.388 & 0.303 & 0.210 & 0.006 & 0.046 & 0.040 & 0.119 & -0.071 \\
\hline & $(0.448)$ & $(0.177)$ & $(0.373)$ & $(0.344)$ & $(0.168)$ & $(0.266)$ & $(0.163)$ & $(0.136)$ \\
\hline \multirow{2}{*}{$\begin{array}{l}\text { Effect for } \\
\text { income } \\
\text { quartile } 4\end{array}$} & 0.519 & 0.438 & 0.133 & 0.187 & -0.178 & -0.127 & -0.074 & -0.187 \\
\hline & $(0.444)$ & $(0.175)^{*}$ & $(0.426)$ & $(0.257)$ & $(0.179)$ & $(0.301)$ & $(0.170)$ & $(0.135)$ \\
\hline \multirow{2}{*}{$\begin{array}{l}\text { Effect on } \\
\text { males }\end{array}$} & -0.035 & 0.009 & 0.077 & -0.150 & -0.199 & -0.170 & -0.202 & -0.084 \\
\hline & $(0.268)$ & $(0.111)$ & $(0.232)$ & $(0.177)$ & $(0.086)^{*}$ & $(0.132)$ & $(0.084)^{*}$ & $(0.072)$ \\
\hline \multirow[t]{2}{*}{ Constant } & 1.904 & 2.320 & -5.722 & -6.448 & -0.939 & -1.869 & -0.459 & \\
\hline & (6.705) & $(3.426)$ & (7.638) & (5.938) & (3.635) & $(4.393)$ & $(3.484)$ & \\
\hline Observations & 4011 & 4011 & 4011 & 4011 & 4011 & 4011 & 4011 & 3624 \\
\hline $\begin{array}{l}\text { F-test SES } \\
\text { interactions }\end{array}$ & 4.36 & 2.23 & 3.88 & 3.05 & 1.34 & 1.75 & 1.37 & 1.38 \\
\hline Prob $>F$ & 0.225 & 0.526 & 0.275 & 0.383 & 0.721 & 0.626 & 0.713 & 0.710 \\
\hline
\end{tabular}


Table 7g: Interaction Effects

\begin{tabular}{|c|c|c|c|c|c|c|c|c|}
\hline & $\begin{array}{l}\text { Run out of } \\
\text { food and } \\
\text { money not } \\
\text { enough } \\
\text { (never-always } \\
\text { [1-4]) }\end{array}$ & $\begin{array}{l}\text { Skip or cut } \\
\text { meals (never- } \\
\text { always [1-4]) }\end{array}$ & $\begin{array}{l}\text { Often eat less } \\
\text { than you felt } \\
\text { you should } \\
\text { (never-always } \\
{[1-4] \text { ) }}\end{array}$ & $\begin{array}{l}\text { Often hungry } \\
\text { (never-always } \\
{[1-4] \text { ) }}\end{array}$ & $\begin{array}{l}\text { Not eat all } \\
\text { day (never- } \\
\text { always [1-4]) }\end{array}$ & $\begin{array}{l}\text { Food from } \\
\text { charity } \\
\text { (never-always } \\
{[1-4] \text { ) }}\end{array}$ & $\begin{array}{l}\text { Spend on } \\
\text { food at home } \\
\text { last week }\end{array}$ & $\begin{array}{l}\text { Spend on } \\
\text { food away } \\
\text { from home }\end{array}$ \\
\hline \multirow[t]{2}{*}{ Wave } & -0.374 & -0.474 & -0.379 & -0.582 & -0.483 & -0.300 & 37.466 & -21.925 \\
\hline & $(0.088)^{* *}$ & $(0.091)^{* *}$ & $(0.091)^{* *}$ & $(0.107)^{* *}$ & $(0.123)^{* *}$ & $(0.145)^{*}$ & (23.899) & (125.735) \\
\hline \multirow[t]{2}{*}{ Valladolid } & -0.180 & -0.099 & -0.091 & -0.185 & -0.242 & -0.237 & -23.690 & -56.710 \\
\hline & $(0.062)^{* *}$ & $(0.063)$ & $(0.062)$ & $(0.071)^{* *}$ & $(0.081)^{* *}$ & $(0.110)^{*}$ & (14.871) & (79.549) \\
\hline \multirow{2}{*}{$\begin{array}{l}\text { Valladolid } \\
\text { wave } 2\end{array}$} & -0.081 & 0.011 & -0.105 & -0.095 & -0.457 & -0.432 & -3.455 & -272.769 \\
\hline & $(0.106)$ & $(0.111)$ & $(0.115)$ & $(0.132)$ & $(0.171)^{* *}$ & $(0.216)^{*}$ & (28.510) & (162.934) \\
\hline \multirow{2}{*}{$\begin{array}{l}\text { Effect for } \\
\text { income } \\
\text { quartile } 1\end{array}$} & -0.085 & -0.124 & -0.092 & -0.173 & 0.072 & 0.218 & -9.592 & -83.965 \\
\hline & (0.139) & $(0.144)$ & $(0.142)$ & $(0.170)$ & $(0.207)$ & $(0.280)$ & (36.313) & (202.370) \\
\hline \multirow{2}{*}{$\begin{array}{l}\text { Effect for } \\
\text { income } \\
\text { quartile } 2\end{array}$} & -0.082 & 0.008 & -0.033 & 0.245 & 0.329 & 0.017 & -4.040 & -361.818 \\
\hline & $(0.140)$ & $(0.138)$ & $(0.151)$ & $(0.164)$ & $(0.203)$ & $(0.240)$ & (36.759) & (275.611) \\
\hline \multirow{2}{*}{$\begin{array}{l}\text { Effect for } \\
\text { income } \\
\text { quartile } 3\end{array}$} & -0.048 & -0.118 & 0.028 & -0.018 & 0.034 & 0.254 & -4.023 & -16.272 \\
\hline & $(0.156)$ & $(0.151)$ & $(0.155)$ & $(0.185)$ & (0.229) & $(0.296)$ & (39.585) & (241.070) \\
\hline \multirow{2}{*}{$\begin{array}{l}\text { Effect for } \\
\text { income } \\
\text { quartile } 4\end{array}$} & -0.170 & -0.308 & -0.171 & -0.057 & -0.199 & 0.220 & 18.048 & 54.166 \\
\hline & (0.149) & $(0.152)^{*}$ & $(0.160)$ & $(0.205)$ & $(0.284)$ & $(0.294)$ & (37.711) & (196.711) \\
\hline \multirow{2}{*}{$\begin{array}{l}\text { Effect on } \\
\text { males }\end{array}$} & -0.226 & -0.130 & -0.117 & -0.277 & -0.098 & -0.120 & 44.613 & -85.944 \\
\hline & $(0.079)^{* *}$ & $(0.083)$ & $(0.084)$ & $(0.100)^{* *}$ & $(0.131)$ & $(0.157)$ & (25.642) & (149.858) \\
\hline Constant & & & & & & & -177.757 & $-5,271.540$ \\
\hline
\end{tabular}




\begin{tabular}{llllllll} 
& & & & $(725.804)$ & $(4,106.503)$ \\
Observations & 3624 & 3619 & 3623 & 3624 & 3625 & 3630 & 3041 \\
$\begin{array}{l}\text { F-test SES } \\
\text { interactions }\end{array}$ & 0.52 & 3.63 & 1.34 & 5.08 & 4.11 & 0.92 & 0.70 \\
Prob>F & 0.915 & 0.304 & 0.720 & 0.166 & 0.250 & 0.821 & 0.925 \\
\hline
\end{tabular}

NOTE: We do not show in this table all the control variables included in the regressions. The reference categories for the control variables are wave 1, Motul, female, do not speak Maya, do not read write a message in Spanish, no schooling, single, and the missing category for respondent income and wealth. SOURCE: Baseline and first follow-up ENCAHEY, 2008 and 2009.

$* *=$ significant at $5 \%$ level of confidence. $*=$ significant at $10 \%$ level of confidence

Table 7h: Interaction Effects

\begin{tabular}{|c|c|c|c|c|c|c|c|c|}
\hline & Total food & $\begin{array}{l}\text { Received free } \\
\text { food (yes-no } \\
[1-0])\end{array}$ & $\begin{array}{l}\text { Eat diary } \\
\text { products: } \\
\text { milk, cheese, } \\
\text { yogurt (at } \\
\text { least once a } \\
\text { day, at least } \\
\text { once a w } \\
\end{array}$ & $\begin{array}{l}\text { Eat eggs, } \\
\text { beans or lentil } \\
\text { (at least once } \\
\text { a day, at least } \\
\text { once a week, } \\
\text { several ti }\end{array}$ & $\begin{array}{l}\text { Eat meat, } \\
\text { poultry or } \\
\text { fish (at least } \\
\text { once a day, at } \\
\text { least once a } \\
\text { week, several } \\
\text { ti }\end{array}$ & $\begin{array}{l}\text { Eat fruit or } \\
\text { vegetables (at } \\
\text { least once a } \\
\text { day, at least } \\
\text { once a week, } \\
\text { several time }\end{array}$ & $\begin{array}{l}\text { Eat tortillas, } \\
\text { bread, } \\
\text { crackers or } \\
\text { other cereals } \\
\text { (at least once } \\
\text { a day, at least } \\
\text { o }\end{array}$ & $\begin{array}{l}\text { Worked for } \\
\text { pay last } \\
\text { month (yes- } \\
\text { no }[1-0])\end{array}$ \\
\hline \multirow[t]{2}{*}{ Wave } & 43.527 & 0.478 & -0.041 & 0.299 & 0.153 & -0.007 & -0.604 & -0.021 \\
\hline & (35.410) & $(0.131)^{* *}$ & $(0.070)$ & $(0.082) * *$ & $(0.081)$ & $(0.074)$ & $(0.146)^{* *}$ & (0.109) \\
\hline \multirow[t]{2}{*}{ Valladolid } & -37.403 & -0.268 & 0.149 & 0.230 & 0.068 & 0.023 & -0.190 & -0.221 \\
\hline & (22.051) & $(0.093) * *$ & $(0.054) * *$ & $(0.059) * *$ & $(0.058)$ & $(0.057)$ & (0.099) & $(0.080) * *$ \\
\hline \multirow{2}{*}{$\begin{array}{l}\text { Valladolid } \\
\text { wave } 2\end{array}$} & -1.245 & -0.078 & -0.026 & -0.036 & -0.165 & -0.001 & 0.273 & 0.371 \\
\hline & $(42.238)$ & $(0.151)$ & $(0.078)$ & $(0.088)$ & $(0.086)$ & $(0.084)$ & $(0.178)$ & $(0.192)$ \\
\hline \multirow{2}{*}{$\begin{array}{l}\text { Effect for } \\
\text { income } \\
\text { quartile } 1\end{array}$} & -26.461 & -0.175 & -0.100 & 0.047 & 0.208 & -0.067 & -0.319 & -0.658 \\
\hline & (53.780) & $(0.180)$ & $(0.103)$ & $(0.110)$ & $(0.104)^{*}$ & $(0.102)$ & $(0.219)$ & $(0.200) * *$ \\
\hline \multirow{2}{*}{$\begin{array}{l}\text { Effect for } \\
\text { income } \\
\text { quartile } 2\end{array}$} & -32.000 & -0.228 & 0.102 & 0.068 & 0.234 & 0.012 & -0.346 & -0.688 \\
\hline & (54.428) & $(0.188)$ & (0.099) & $(0.107)$ & $(0.106)^{*}$ & $(0.105)$ & $(0.224)$ & $(0.196) * *$ \\
\hline
\end{tabular}




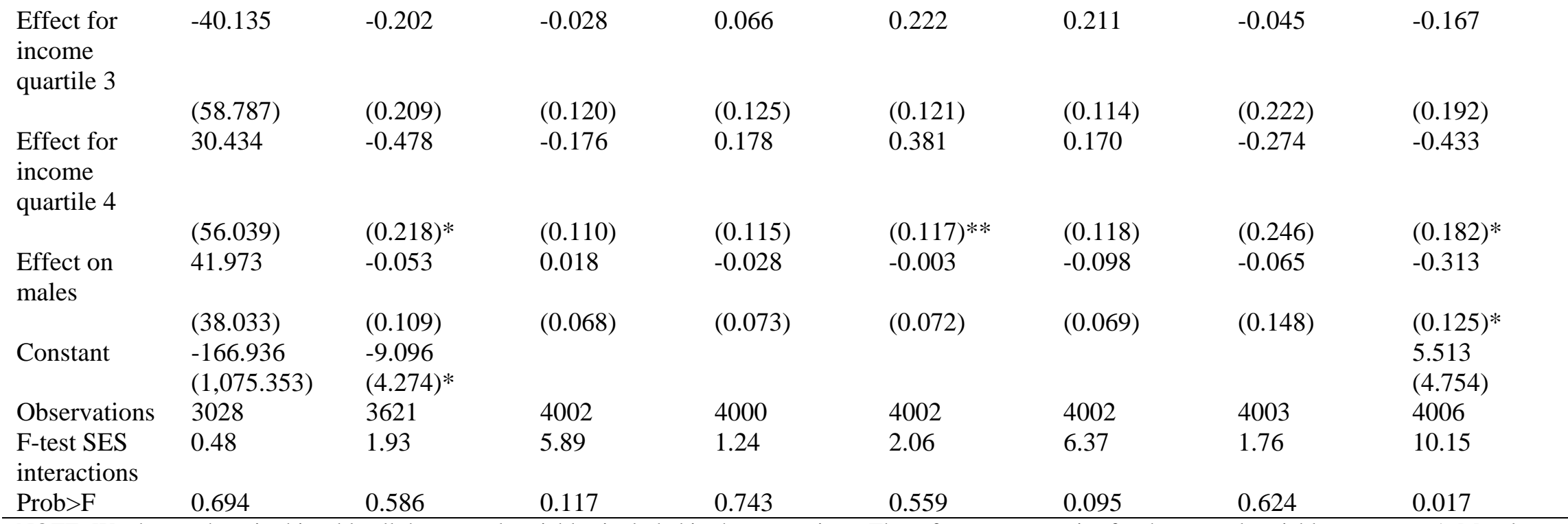

NOTE: We do not show in this table all the control variables included in the regressions. The reference categories for the control variables are wave 1, Motul, female, do not speak Maya, do not read write a message in Spanish, no schooling, single, and the missing category for respondent income and wealth. SOURCE: Baseline and first follow-up ENCAHEY, 2008 and 2009.

$* *=$ significant at $5 \%$ level of confidence. $*=$ significant at $10 \%$ level of confidence

Table 8.- Pre-treatment Characteristics of Treatment and Control Towns

\begin{tabular}{lcc}
\hline \multicolumn{1}{c}{ Variable } & $\begin{array}{c}\text { Treatment Town } \\
\text { (Valladolid) }\end{array}$ & $\begin{array}{c}\text { Control Town } \\
\text { (Motul) }\end{array}$ \\
\hline $\begin{array}{l}\text { Illiterate population 15 } \\
\text { years old or above (\%) }\end{array}$ & 10.95 & 11.23 \\
$\begin{array}{l}\text { Population 15 years old or } \\
\text { above with incomplete } \\
\text { primary }\end{array}$ & 27.34 & 34.46 \\
Households without sewage & 9.60 & 23.76
\end{tabular}


or toilet (\%)

Households without

2.15

electricity (\%)

Households without piped

5.77

10.29

water (\%)

Households with floor of

3.63

2.96

earth (\%)

Households without fridge

24.98

28.39

(\%)

Level of poverty

Low

Medium

Poverty index

$-1.10$

$-0.92$

Inhabitants

45,868

21,508

NOTE: Estimations conducted by the Mexican National Population Council (CONAPO) based on the 2005 Mexican Census, INEGI, Mexico, 2005.

SOURCE: CONAPO (2005). 
Table 9.- Difference-in-Differences using Parametric and Non-Parametric Methods

\begin{tabular}{|c|c|c|c|}
\hline $\begin{array}{l}\text { Variable (verbal scale [numeric } \\
\text { codes]) }\end{array}$ & $\begin{array}{l}\text { Dif-in-dif of } \\
\text { the means }\end{array}$ & $\begin{array}{l}\text { Dif-in-dif } \\
\text { Regressions }\end{array}$ & $\begin{array}{l}\text { Dif-in-dif propensity score } \\
\text { matching }\end{array}$ \\
\hline Self-reported health (excellent, very & & 0.085 & \\
\hline \multirow[t]{2}{*}{ good, good, fair, poor [1-5]) } & 0.086 & & 0.104 \\
\hline & $(0.037)^{* *}$ & $(0.0037)^{*}$ & $(0.042)^{* *}$ \\
\hline $\begin{array}{l}\text { Subjective mortality expectation } \\
\text { (chances to live at least } 10 \text { years more }\end{array}$ & & -3.107 & \\
\hline \multirow{2}{*}[0-100]{)} & 1.010 & & 0.306 \\
\hline & $(2.380)$ & $(2.219)$ & (2.489) \\
\hline $\begin{array}{l}\text { Feel sad, blue or depressed for } 2 \\
\text { weeks more during the past } 3 \text { months }\end{array}$ & & -0.018 & \\
\hline \multirow[t]{2}{*}{ (yes-no[1-0]) } & -0.024 & & -0.005 \\
\hline & $(0.033)$ & $(0.034)$ & $(0.033)$ \\
\hline \multirow[t]{2}{*}{$\begin{array}{l}\text { Satisfied relation fam. members (very } \\
\text { satisfied-very dissatisfied [1-5]) }\end{array}$} & 0.037 & 0.046 & 0.015 \\
\hline & $(0.041)$ & $(0.041)$ & $(0.043)$ \\
\hline \multirow[t]{2}{*}{$\begin{array}{l}\text { Satisfied hh income(very satisfied- } \\
\text { very dissatisfied [1-5]) }\end{array}$} & -0.074 & -0.049 & -0.073 \\
\hline & $(0.052)$ & $(0.053)$ & $(0.056)^{*}$ \\
\hline \multirow[t]{2}{*}{$\begin{array}{l}\text { Satisfied social contacts (very } \\
\text { satisfied-very dissatisfied [1-5]) }\end{array}$} & 0.012 & 0.031 & 0.005 \\
\hline & $(0.043)$ & $(0.044)$ & $(0.047)$ \\
\hline \multirow[t]{2}{*}{$\begin{array}{l}\text { Satisfied with job (very satisfied-very } \\
\text { dissatisfied [1-5]) }\end{array}$} & 0.002 & 0.021 & 0.018 \\
\hline & $(0.044)$ & $(0.045)$ & $(0.047)$ \\
\hline \multirow[t]{2}{*}{$\begin{array}{l}\text { Satisfied with health (very satisfied- } \\
\text { very dissatisfied [1-5]) }\end{array}$} & -0.095 & -0.092 & -0.055 \\
\hline & $(0.050)^{*}$ & $(0.051)$ & $(0.052)$ \\
\hline $\begin{array}{l}\text { Satisfied life in general (very } \\
\text { satisfied-very dissatisfied [1-5]) }\end{array}$ & $\begin{array}{r}-0.041 \\
(0.041)\end{array}$ & $\begin{array}{r}-0.044 \\
(0.041)\end{array}$ & $\begin{array}{r}-0.027 \\
(0.041)\end{array}$ \\
\hline
\end{tabular}


Number of acute conditions

Often Pain (yes-no[1-0])

How strong is the pain (mild, moderate, severe $[1,2,3])$

Number of chronic conditions

Number of ADL's

Number of IADL's

Smoke now (yes-no [1-0])

Number of cigarettes in a day

Drink alcoholic beverages (yes-no[1-

0])

Number of days a week drinks alcoholic beverages

Number of drinks per day

Visited doctor (yes-no[1-0])

Number of doctor visits

Visited a folk healer (yes-no[1-0])

$\begin{array}{rr}-0.039 & -0.035 \\ (0.019)^{* *} & (0.020) \\ 0.067 & 0.070 \\ (0.026)^{* *} & (0.027)^{*} \\ & 0.012 \\ 0.003 & (0.061) \\ (0.060) & 0.028 \\ 0.025 & (0.046) \\ (0.044) & 0.139 \\ -0.177 & (0.171) \\ (0.164) & 0.018 \\ 0.004 & (0.052) \\ (0.051) & 0.004 \\ 0.004 & (0.006) \\ (0.006) & 0.068 \\ 0.065 & (0.035) \\ (0.033)^{* *} & -0.161 \\ -0.161 & (0.025)^{* *} \\ (0.024)^{* *} & -0.075 \\ & \\ -0.052 & (0.042) \\ (0.036) & -0.135 \\ -0.138 & (0.063) \\ (0.060)^{* *} & 0.083 \\ 0.088 & (0.029)^{* *} \\ (0.027)^{* *} & 0.274 \\ 0.286 & (0.115)^{*} \\ (0.112)^{* *} & -0.013 \\ -0.017 & \\ & \end{array}$

$-0.027$

$(0.021)^{*}$

0.076

$(0.027)^{* *}$

0.031

(0.061)

0.025

$(0.048)$

$-0.152$

( 0.171)

0.000

(0.054)

0.002

( 0.006)

0.069

$(0.033)^{* *}$

$-0.159$

$(0.026)^{* *}$

$-0.063$

$(0.034)^{* *}$

$-0.125$

$(0.058)^{* *}$

0.082

$(0.029)^{* *}$

0.295

$(0.121)^{* *}$

$-0.015$ 
Number of folk healer visits

Visited a dentist (yes-no[1-0])

Number of dentist visits

Outpatient procedures (yes-no[1-0])

Number of outpatient procedures (ambulatory surgery)

Consulted a pharmacist (yes-no[1-0])

Bought no medicines because they are too expensive (yes-no[1-0])

Pay out-of-pocket (oop) medical cost or medications (yes-no[1-0])

OOP expenses paid by relatives (yesno[1-0])

OOP expenses paid by elderly eligible (yes-no[1-0])

Serious health problem but did not go to the doctor (yes-no[1-0])

Did not go to the doctor because of money (yes-no[1-0])

\begin{tabular}{|c|c|c|}
\hline$(0.011)$ & (0.011) & $(0.011)^{*}$ \\
\hline-0.044 & -0.034 & -0.029 \\
\hline$(0.030)$ & $(0.030)$ & $(0.030)$ \\
\hline-0.015 & -0.017 & -0.022 \\
\hline$(0.015)$ & $(0.016)$ & $(0.015)^{*}$ \\
\hline-0.050 & -0.059 & -0.066 \\
\hline (0.039) & $(0.040)$ & $(0.041)^{*}$ \\
\hline-0.003 & -0.004 & -0.003 \\
\hline \multirow[t]{2}{*}{$(0.006)$} & $(0.006)$ & $(0.007)$ \\
\hline & -0.004 & \\
\hline-0.007 & & -0.007 \\
\hline$(0.010)$ & $(0.006)$ & $(0.010)$ \\
\hline-0.001 & 0.004 & -0.002 \\
\hline \multirow[t]{2}{*}{$(0.014)$} & $(0.014)$ & (0.015) \\
\hline & -0.051 & \\
\hline-0.060 & & -0.049 \\
\hline \multirow[t]{2}{*}{$0.022)^{* *}$} & $(0.023)$ & $(0.022) * *$ \\
\hline & 0.006 & \\
\hline 0.006 & & 0.003 \\
\hline \multirow[t]{2}{*}{$(0.028)$} & $(0.030)$ & $(0.030)$ \\
\hline & -0.110 & \\
\hline-0.111 & & -0.106 \\
\hline \multirow[t]{2}{*}{$(0.022)^{* *}$} & $(0.023) * *$ & $(0.025)^{* *}$ \\
\hline & 0.086 & \\
\hline 0.085 & & 0.083 \\
\hline \multirow[t]{2}{*}{$(0.024)^{* *}$} & $(0.025)^{* *}$ & $(0.026)^{* *}$ \\
\hline & -0.024 & \\
\hline-0.029 & & -0.030 \\
\hline \multirow[t]{2}{*}{ (0.019) } & (0.019) & $(0.020)^{*}$ \\
\hline & -0.014 & \\
\hline-0.016 & & -0.018 \\
\hline$(0.015)$ & $(0.016)$ & $(0.015)$ \\
\hline
\end{tabular}


Relatives or friends pay your

expenses (not true, sometimes true,

often true, always true [1-4])

Feel a burden on your family or

friends (not true, sometimes true,

often true, always true [1-4])

Activities you used to do but can't do

because of lack of money (yes-no[1-

Donation to the church but can't do

Donation to local parties but can't do

Donation to family or friends parties

Community activities but can't do due 
Help out non-relatives but can’t do due to money (yes-no[1-0])

Travel to visit family or friends but can't do due to money (yes-no[1-0])

Sometimes we do not have enough to eat (yes-no[1-0])

Often we do not have enough to eat (yes-no[1-0])

Sometimes or often we don't have enough to eat because of money (yesno[1-0])

Often worried run out of food last three months (never-always [1-4]))

Often run out of food last three months (never-always [1-4]))

Skip or cut meals (never-always [14])

\begin{tabular}{|c|c|c|}
\hline$(0.020)$ & $(0.021)$ & $(0.021)$ \\
\hline-0.006 & -0.004 & -0.004 \\
\hline (0.008) & (0.008) & $(0.008)$ \\
\hline-0.018 & -0.020 & -0.018 \\
\hline$(0.011)^{*}$ & $(0.012)$ & $(0.011)^{*}$ \\
\hline $\begin{array}{r}-0.021 \\
(0.026)\end{array}$ & $\begin{array}{r}-0.010 \\
(0.031)\end{array}$ & $\begin{array}{r}-0.019 \\
(0.027)\end{array}$ \\
\hline 0.024 & 0.026 & 0.022 \\
\hline$(0.013)^{*}$ & $(0.016)$ & $(0.014)^{*}$ \\
\hline 0.038 & $\begin{array}{r}0.035 \\
(0.030)\end{array}$ & $\begin{array}{r}0.026 \\
(0.026)\end{array}$ \\
\hline $\begin{array}{r}0.067 \\
(0.053)\end{array}$ & $\begin{array}{r}0.116 \\
(0.061)\end{array}$ & $\begin{array}{r}0.060 \\
(0.056)\end{array}$ \\
\hline $\begin{array}{r}-0.146 \\
.046) * *\end{array}$ & $\begin{array}{r}-0.139 \\
(0.052)^{* *}\end{array}$ & $\begin{array}{r}-0.134 \\
(0.047)^{* *}\end{array}$ \\
\hline & -0.071 & \\
\hline $\begin{array}{r}-0.075 \\
(0.045)^{*}\end{array}$ & $(0.050)$ & $\begin{array}{r}-0.072 \\
(0.047)^{*}\end{array}$ \\
\hline
\end{tabular}


Often eat less than you felt you

should (never-always [1-4])

Often hungry (never-always [1-4])

$-0.100$

$-0.099$

$(0.042)^{* *}$

$(0.047)^{*}$

$-0.103$

$-0.092$

Not eat all day (never-always [1-4])

$(0.034)^{* *}$

$(0.038)^{*}$

$-0.121$

$-0.100$

$(0.025)^{* *}$

$(0.028)^{* *}$

Food from charity (never-always [1-

$-0.050$

4])

$-0.057$

$(0.019)^{* *}$

Spend on food at home last week

$(0.018)^{* *}$

8.730

18.869

(20.600)

(22.229)

Spend on food away from home

$-14.100$

$-11.726$

(13.000)

(17.510)

$-15.100$

11.128

(34.100)

(37.975)

0.045

$-0.051$

$(0.021)^{* *}$

(0.023)*

Eat diary products: milk, cheese,

yogurt (at least once a day, at least once a week, several times a month, once in a while, never [1-5])

Eat eggs, beans or lentil (at least once

a day, at least once a week, several times a month, once in a while, never [1-5])

Eat meat, poultry or fish (at least once 
times a month, once in a while, never [1-5])

Eat fruit or vegetables (at least once a $(0.060)$ $(0.064)$

$(0.063)$ day, at least once a week, several

times a month, once in a while, never [1-5])

$-0.041$

$-0.007$

$-0.025$

Eat tortillas, bread, crackers or other $(0.064)$

(0.069)

$(0.069)$

cereals (at least once a day, at least once a week, several times a month, once in a while, never [1-5])

0.002

$-0.011$

$(0.027)$

$(0.027)$

(0.027)

Worked for pay, last month (yes-

$-0.043$

$-0.045$

$(0.016)^{* *}$

0.293

$(0.016)^{* *}$

0.244

$-0.043$

Immediate recall (number of words)

$(0.083)^{* *}$

$(0.089)^{* *}$

Delayed recall (number of words)

0.609

0.547

$(0.102)^{* *}$

$(0.109)^{* *}$

$(0.015)^{* *}$

0.303

$(0.090)^{* *}$

0.608

$(0.099)^{* *}$

Feel fear someone robbing you

(never, sometimes, usually, always [1-4])

$\begin{array}{rrr}-0.036 & -0.005 & -0.038 \\ (0.053) & (0.053) & (0.055) \\ & & \\ 0.013 & 0.004 & 0.004 \\ (0.037) & (0.037) & (0.040)\end{array}$

Feel fear someone close to you will take your money (never, sometimes, usually, always [1-4]) 
Money in a safe place (yes-no [1-0])

\begin{tabular}{llc}
-0.004 & -0.006 & -0.004 \\
$(0.010)$ & $(0.010)$ & $(0.010)$ \\
& & \\
-0.018 & -0.017 & -0.034 \\
$(0.029)$ & $(0.028)$ & $(0.031)$ \\
\hline
\end{tabular}

Feel verbally or physically abused (never, sometimes, usually, always

[1-4])

(0.029)

NOTE: The reference categories are wave 1, Motul, female, do not speak Maya, do not read write a message in Spanish, no schooling, and single. SOURCE: Baseline and first follow-up ENCAHEY, 2008 and 2009.

$* *=$ significant at $5 \%$ level of confidence. $*=$ significant at $10 \%$ level of confidence 
Table 10: Sample Baseline and Follow-up Valladolid and Motul

\begin{tabular}{lccc}
\hline & Valladolid & Motul & Total \\
\hline Baseline survey & 1,264 & 956 & 2,220 \\
First follow-up & 1,159 & 859 & 2,018 \\
Attrition & & & \\
Total & 105 & 97 & 202 \\
Died & 64 & 57 & 121 \\
Refused & 9 & 12 & 21 \\
Could not be & 32 & 28 & 60 \\
contacted & & & \\
\hline
\end{tabular}

SOURCE: Baseline and first follow-up ENCAHEY, 2008 and 2009. 